\title{
Relations between measures of attention and memory in the assessment of children with attentional difficulties
}

Karen S. Kirk

West Virginia University

Follow this and additional works at: https://researchrepository.wvu.edu/etd

\section{Recommended Citation}

Kirk, Karen S., "Relations between measures of attention and memory in the assessment of children with attentional difficulties" (1999). Graduate Theses, Dissertations, and Problem Reports. 1040.

https://researchrepository.wvu.edu/etd/1040

This Dissertation is protected by copyright and/or related rights. It has been brought to you by the The Research Repository @ WVU with permission from the rights-holder(s). You are free to use this Dissertation in any way that is permitted by the copyright and related rights legislation that applies to your use. For other uses you must obtain permission from the rights-holder(s) directly, unless additional rights are indicated by a Creative Commons license in the record and/ or on the work itself. This Dissertation has been accepted for inclusion in WVU Graduate Theses, Dissertations, and Problem Reports collection by an authorized administrator of The Research Repository @ WVU.

For more information, please contact researchrepository@mail.wvu.edu. 


\title{
Relations Between Measures of Attention and Memory in the
} Assessment of Children with Attentional Difficulties

\author{
Karen S. Kirk
}

\author{
Dissertation submitted to the \\ Eberly College of Arts and Sciences \\ at West Virginia University \\ in partial fulfillment of the requirements \\ for the degree of

\section{Doctor of Philosophy} \\ in \\ Child Clinical Psychology
Jennifer S. Haut, Ph.D., A.B.P.P., Chair
Marc W. Haut, Ph.D., A.B.P..P.
Andrew S. Bradlyn, Ph.D.
Barry Edelstein, Ph.D.
Stanley Cohen, Ph.D.
Department of Psychology
Morgantown, West Virginia
1999

Keywords: Attention, Learning, Memory, Working Memory, Gordon Diagnostic System (GDS), Wide Range Assessment of Memory and Learning (WRAML)

\section{Copyright 1999 Karen S. Kirk}




\section{ABSTRACT \\ Relations Between Measures of Attention and Memory in the Assessment of Children with Attentional Difficulties}

Karen S. Kirk

Attentional difficulties are associated with a number of different pediatric clinical disorders, including attention deficit hyperactivity disorder (ADHD), learning disabilities (LD), and traumatic brain injury (TBI). Further, two cognitive functions dependent upon attention, learning and memory, are characteristic deficits in LD children, and have been documented as correlated weaknesses in ADHD and TBI populations. However, few investigations have examined the relation between these cognitive functions, in terms of understanding the respective contributions of attention, learning, and memory deficits in the clinical groups indicated above. In the present study, a measure of attention, the Gordon Diagnostic System (GDS) was compared to a measure of learning and memory in children, the Wide Range Assessment of Memory and Learning (WRAML), in ADHD, LD/ADHD, TBI, and typicallyfunctioning Control groups (aged 9-14 inclusive, $\underline{N}=25$ per group). The purposes of this comparison were (a) to determine the correlation between the GDS and learning and memory subtests thought to have a strong attentional component, and (b) to examine how performance profiles on the various measures would discriminate among clinical groups, and predict group classification. Results of the correlational analysis indicated relations among the measures along the lines of visual and verbal working memory, versus the expected attention/memory dichotomy. The discriminant analysis revealed that the LD/ADHD group demonstrated significantly more difficulties on both verbal and visual working memory tasks, compared to the other groups, and that visual working memory tasks primarily separated the ADHD and TBI groups from the Control group. These findings were discussed in terms of the importance of considering working memory as a significant factor in attentional functions of children with ADHD, LD, and TBI. 


\section{Attention and Memory in Children DEDICATION

This dissertation project is dedicated to the memory of my maternal grandmother,

\section{Dorothy Dale Wharton Tomer}

who always wanted to see "Ph.D." after my name on the office door. It's there now. 


\section{ACKNOWLEDGMENTS}

I would like to extend my sincerest appreciation and gratitude to my Dissertation Committee Members, who supported and guided me through the completion of this project. Dr. Jennifer S. Haut, my Committee Chair, offered her unique expertise regarding the content of the project as well as the mechanics of completing a dissertation, and most importantly, provided calm, reassuring mentorship during even the most frustrating of moments. Dr. Marc Haut served the role of an expert teacher in the field of neuropsychology, in a very supportive and helpful manner. Dr. Andrew Bradlyn's proficient knowledge about pediatric populations with ADHD and related learning disorders was very beneficial in keeping me on the "cutting edge" of pertinent information in the field. Dr. Stanley Cohen guided me through the mire of statistical questions and analyses involved in this project, and eventually led me to admit that I actually enjoyed learning about those quantitative methods! Finally, Dr. Barry Edelstein has lent invaluable mentoring to me throughout my graduate career at WVU, serving not only as expert teacher but also as an esteemed role model regarding both professional and personal behaviors. I am indebted to each of these people for helping me to complete this final project of the doctoral program, so that I can realize a goal that I set for myself many years ago.

Other members of the Department of Psychology at West Virginia University have also helped to propel me toward my dream. Among all of the wonderful teachers under whom I studied and learned, I especially want to thank Dr. Daniel McNeil, Clinical Training Director, for gently, but firmly, pushing me to remember deadlines; and Dr. Joseph Scotti for helping to bring me into the Child Clinical Program and for providing countless opportunities to enhance my professional growth. I also want to express appreciation to Mrs. Debra Swinney, Office of Graduate Studies, for her reliable assistance and support at every step along the way of this process.

Finally, to my family, who has supported me with gifts of love and life wisdom, I am deeply grateful. My parents, Wilber and Dolores Kirk, my great-aunt, Ethel Cox, my sister, Kelly Oxendine, and my cousins, Jeff and Sherri Planton endured countless telephone calls and visits to support me, especially on the more frustrating days. My husband, Travis Williams, has provided admirable tolerance and support over the course of the dissertation completion process. To each of these persons, I am certain that I would not have completed this whole effort without your support and kindnesses. 


\section{TABLE OF CONTENTS}

Dedication

Acknowledgments

List of Tables

List of Figure Captions

List of Figures

Introduction

Definitions of attention

Theories regarding attention deficits

Relation between attention and learning

Relation between attention and memory

Conceptualizations of memory

Developmental aspects of memory

Implications of the relations between attention, learning, and memory

Gordon Diagnostic System (GDS)

Assessment of learning and memory

Wide Range Assessment of Memory and Learning (WRAML)

Purpose of the present study

Reasons to further investigate utility of the GDS and WRAML

Research questions
Assessment of attentional behaviors

PAGE

iii

iv

vii

viii

ix

1

4

8

13

17

18

22

25

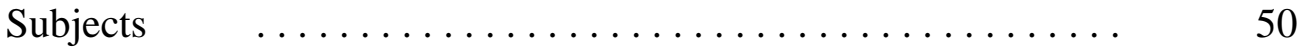

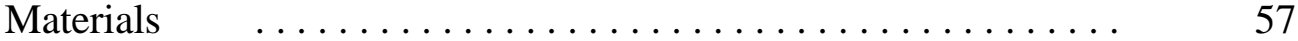

Wide Range Assessment of Memory and Learning (WRAML) 57

Technical aspects of the WRAML $\ldots \ldots \ldots \ldots \ldots \ldots . \ldots 6$

Gordon Diagnostic System (GDS) $\quad \ldots \ldots \ldots \ldots \ldots \ldots \ldots$

Technical aspects of the GDS $\quad \ldots \ldots \ldots \ldots \ldots \ldots . \ldots \ldots$

Other neuropsychological measures $\ldots \ldots \ldots \ldots \ldots \ldots . \ldots \ldots$

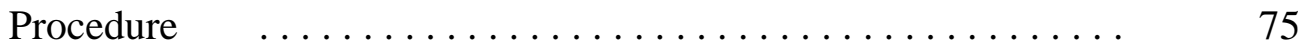

Data Analysis . . . . . . . . . . . . . 77

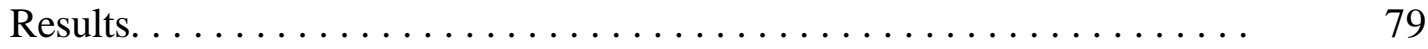

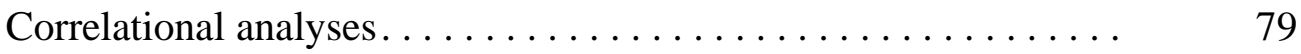

Direct discriminant function analysis . . . . . . . . . . 85 


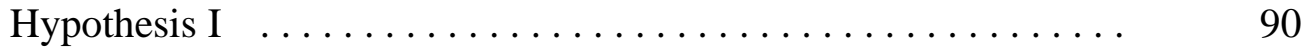

Hypothesis II .......................... 97

Limitations of the study and implications for further research. . . . 103

Conclusions ......................... 105

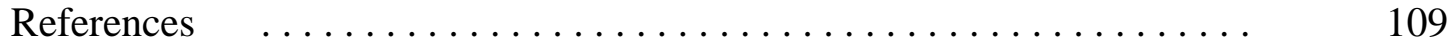

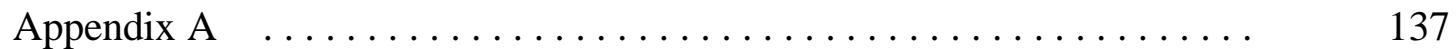




\section{LIST OF TABLES}

\section{TABLE}

1

2

3

4

5

6

7

8

9

10

11

12

13

14
PAGE

140

141

142

143

144

145

146

147

148

149

150

151

152

153 
Attention and Memory in Children

LIST OF FIGURE CAPTIONS

viii

PAGE

FIGURE 1

154 
Attention and Memory in Children

LIST OF FIGURES

FIGURE 1

PAGE

155 


\section{Introduction}

Attention deficit disorder, with or without hyperactivity (ADD or ADD-H), was initially described as a distinct psychiatric diagnosis in the third edition of the Diagnostic and Statistical Manual of Mental Disorders (DSM-III; American Psychiatric Association (APA), 1980). Since then, a flood of scientific and practical information has lent varying perspectives on the diagnostic significance of the three core characteristics of this disorder: inattention, impulsivity, and overactivity. The changing configurations of symptoms in the DSM revisions is a case in point. In 1987, the DSM-III-R (APA, 1987) indicated a shift in the structuring of diagnostic subtypes to a primary category of Attention-Deficit Hyperactivity Disorder (ADHD), with a "rule-out" criteria of no impulsive or overactive symptoms for the "undifferentiated" subtype (akin to the previous ADD without hyperactivity). The newest version of the manual of psychiatric disorders (DSM-IV; APA, 1994) allows for diagnosis along two dimensions: inattention, versus impulsivity and hyperactivity. If enough diagnostic criteria are met for both of the subtypes, the descriptor of "combined type" can be applied. The validity of these three subtypes has been supported in several studies of referred and nonreferred samples of children (Baumgaertel, Wolraich, \& Dietrich, 1995; Gaub \& Carlson, 1997; Lahey et al., 1994; Wolraich, Hannah, Pinnock, Baumgaertel, \& Brown, 1996).

These variations in diagnostic classification and subtyping have resulted in confusion about the array of symptoms that must be addressed in attempting to differentially diagnosis individuals with ADHD (Armstrong, 1996; Fletcher, Morris, \& Francis, 1991; Halperin et al., 1990; Halperin, Matier, Bedi, Sharma, \& Newcorn, 1992; Newcorn et al., 1994). To further complicate this process, a number of other neurological, psychiatric, and learning disorders involve characteristic deficits in attentional functioning; thus it becomes critical to be able to 
identify the specific aspects of "attending" that may discriminate among various disorders and performance difficulties. Examples of neurological disorders with related attentional deficits include seizure disorders, Tourette's syndrome, traumatic brain injury (TBI), and central nervous system neuropathies such as tumors; psychiatric disorders include mood and anxiety problems. The broad array of learning disabilities (LDs) represents processing and learning difficulties that typically involve related attentional dysfunction. In the present study, two of these clinical groups, children with TBI and children with comorbid LD and ADHD, were compared to children with "pure" ADHD in order to investigate various aspects of attentional deficits. Traumatic brain injury in children has consistently been linked to attentional difficulties (Dennis, Wilkinson, Koski, \& Humphreys, 1995; Kaufmann, Fletcher, Levin, Miner, \& EwingCobbs, 1993; Max et al., 1998; Parker, 1994; Whyte et al., 1996). In the TBI population, the nature of the attention problems is related to the site(s) and severity of the brain injury, as well as to the age of the child when the injury occurred (Dennis et al., 1995; Fletcher, Ewing-Cobbs, Francis, \& Levin, 1995). The neuroanatomical sites most susceptible to brain trauma are the frontal and temporal lobes (Bruce, 1995; Dennis et al., 1995); dysfunction in those areas is also related to attentional anomalies. (This topic is discussed in further detail below, in relation to ADHD.) An interesting speculation is that the difficulties in attentional functioning experienced by children with a history of frontal or temporal lobe TBI are related to structural abnormalities, while the deficits demonstrated by children with ADHD may be more related to functional disorders, such as anomalies in neurotransmitter action. Although neurobiological testing would have to be undertaken to provide specific answers to those etiology questions, it should also be valuable to investigate whether the similar "practical" presentations of attentional problems among the two groups can be sorted out on neuropsychological tests. 
The LD group was included in the present study due to the documented high rate of coexisting learning disabilities and ADHD. In the LD population, it has been estimated that 40$80 \%$ of individuals also demonstrate attentional difficulties (Epstein, Shaywitz, Shaywitz, \& Woolston, 1991). Alternately, reports have indicated that approximately 20-40\% of ADHD children have diagnosable LDs (August \& Garfinkel, 1989; Barkley, 1998; Korkman \& Pesonen, 1994; Semrud-Clikeman et al., 1992), with some estimates even as high as $80 \%$ or more of the ADHD population (McGee \& Share, 1988). These prevalence estimates, of course, depend on the criteria used for diagnosis of both ADHD and LD. The shared comorbidity across these two clinical groups raises questions about similarities in neurobiological processes related to attention, learning, and memory. Indeed, it is often very difficult to determine the relative contributions and directionality of deficits in attention, impulsivity, distractibility, informationprocessing, memory, and response planning and production. Again, neuropsychological testing may provide some insights regarding these cognitive processes.

Taken together then, knowledge about particular cognitive and behavioral functions of children in the three identified clinical groups should add to an understanding of their respective attentional and learning difficulties. Such information should also be helpful for appropriate determination of diagnosis, prognosis, and treatment planning. These issues and questions provided the impetus for the present investigation. The following review first summarizes current theories regarding the attentional difficulties demonstrated by individuals with ADHD and related disorders, and then focuses on the relation of those deficits to learning and to memory. This discussion will lead to a description of neuropsychological tests used to measure those cognitive abilities in children, with special emphasis on reports involving pediatric ADHD, 
LD, and TBI populations. Finally, the purpose of the present study is elaborated, with a description of specific research questions.

\section{Definitions of attention}

It is important to recognize at the outset that one of the most difficult tasks in determining the particular functions of attention has been in operationally defining inattention, distractibility, impulsivity, and hyperactivity (Carlson \& Rapport, 1989). The cognitive and behavioral aspects and contributions of each of these "symptoms" to the clinical description of this disorder have yet to be determined conclusively (Barkley, 1990; Halperin et al., 1992; Rapport, 1992; Tryon, 1993). Barkley (1990) suggested functional definitions of attention and related behaviors. Thus, in the behavior analytic tradition (e.g., Skinner, 1953), the characteristic problems associated with ADHD can be described as a relation between environmental events and an individual's behavior. Attention can be measured in terms of responses: if a child responds to an event (i.e., a discriminative stimulus), then he "attended" to it. If he did not respond, he likely did not "attend." If he does not wait for a stimulus (usually an expected or continuous one) to occur, then he is described as "impulsive." If the presence of a number of additional stimuli reduces the individual's responses to the discriminative stimulus, then he is characterized as "distractible." Although these definitions may seem oversimplified, they provide a starting point from which to think about attention.

Researchers in cognitive-behavioral, neuropsychology, and neuroscience fields have addressed finer differentiations of attending, such as selective versus divided attention, and identification of the neural mechanisms for producing attentive behaviors (Colby, 1991; Posner \& Petersen, 1990; Posner \& Rafal, 1987; van Zomeren \& Brouwer, 1994; Whyte, 1992). Although it is beyond the scope of this project to discuss those aspects of attention, it is 
important to consider some of the cognitive functions that are dependent on attention. For example, the cognitive processes of learning and memory are closely related to attending skills (August \& Garfinkel, 1990; Blondis, Accardo, \& Snow, 1989; Korkman \& Pesonen, 1994; O'Neill \& Douglas, 1996; Wolfe, 1996). Children who demonstrate attentional deficits are often described as "knowing" a fact or a concept one day, but not the next, which suggests encoding and recall problems. Further, psychoeducational profiles of children with ADHD often reflect specific cognitive and achievement deficits, which raises questions regarding the contributions of attention problems versus memory-based learning disabilities (Blondis et al., 1989). Individuals who have sustained a TBI also exhibit learning and memory difficulties, in addition to attention problems, as measured on both everyday tasks (Parker, 1994; Whyte et al., 1996) and on standardized tests (Farmer et al., 1999; Kaufmann et al., 1993; Yeates, Blumenstein, Patterson, \& Delis, 1995). Clearly, attention can be viewed and defined from a number of different perspectives, with learning and memory being two significant cognitive functions directly affected by attentional disorders.

Probably the most significant development in considering the nature of attention and its relation to learning and memory has been the inception of a "working memory" model. Baddeley (1986) defined working memory as "... a system for the temporary holding and manipulation of information during the performance of a range of cognitive tasks such as comprehension, learning, and reasoning" (p.34). Thus working memory is thought to effect a bridge between attention and memory, by providing a "workspace" for holding and rehearsing information (despite any intervening, incoming stimuli), adapting or adjusting information as required by task demands, and recalling the information within a brief, if not immediate, time 
frame. The developmental aspects of working memory, and its functions in children's cognitive processes, has been studied from several different perspectives.

First, developmental literature suggests that working memory functions progress dimensionally, from refinement of basic sensory and perceptual abilities to maturation of neural networks that incorporate the many cognitive processes involved in mentally manipulating and remembering information (Luciana \& Nelson, 1998; Swanson, 1996). Supporting evidence includes developmental changes in the ability to effectively sort through information in working memory, and to inhibit the use of irrelevant information (Lorsbach \& Reimer, 1997). Further, research suggests that those neural activities required to fluently perform working memory activities are not fully-developed until 8-10 years of age (Hale, Bronik, \& Fry, 1997; Luciana \& Nelson, 1998).

Second, recent research has supported a dichotomous separation of working memory into visual and verbal subsystems (Baddeley, 1998), with developmental implications for interaction and shifting among those processes (Fastenau, Conant, \& Lauer, 1998). For example, children initially rely more strongly on visual-spatial processing of written symbols, but later begin to integrate verbal processes for learning and recalling written information, such as when they learn sounds for alphabet letters, and begin to read. Investigations regarding the task-specific versus integrated involvement of the various subsystems of working memory, particularly on specific activities performed by children, are just beginning to appear in the literature.

Third, there continues to be some debate about whether working memory and short-term memory are synonymous (Sroufe, Cooper, \& DeHart, 1996), or whether they involve different cognitive processes. Indeed, many neuropsychological investigations regarding working memory have utilized tasks that have traditionally been thought to measure attention and/or 
short-term memory (Casey et al., 1995; Hale et al., 1997; Karatekin \& Asarnow, 1998; Swanson, 1996). However, some researchers have suggested that measurements of short-term memory do not reflect all of the processes involved in working memory (Halford, Maybery, O'Hare, \& Grant, 1994). According to this argument, immediate recall tasks, with no intervening or additional information presented, would be considered measures of attention and short-term memory. Alternately, working memory tasks would measure processing capacity: dual-task paradigms, for example, assess the individual's ability to hold and manipulate one set of information while a second task is presented, and then recall the first set of information. Although it is beyond the scope of this paper to examine this measurement issue in depth, it is important to be aware of the apparent lack of concensus regarding these terms and concepts, and how each is measured.

Finally, evidence for frontal lobe activity related to working memory processes has been documented in both adult and pediatric populations (Casey et al., 1995; Luciana \& Nelson, 1998; Shallice, 1982). These findings are significant in thinking about the role of working memory for children with ADHD and related disorders. Shallice (1982), for example, suggested that frontal lobe dysfunction is associated with planning impairments, as evidenced in appropriate performance on routine tasks versus impaired execution of new and unfamiliar tasks by persons with frontal lobe anomalies. Clinical observations of children with ADHD typically indicate similar difficulties in planning and performing new routines.

New conceptualizations of ADHD do, in fact, consider working memory to be a critically-affected executive function for individuals with this disorder (Barkley, 1997, 1998), as described in the next section. Further, neuroscience evidence is presented which supports the involvement of frontal lobe anomalies in the deficits demonstrated by children with ADHD. 
These findings are then discussed in terms of a broader view of ADHD, incorporating than the "traditional" concepts of sustained attention and impulsivity, and additionally considering the role of working memory.

Theories regarding attention deficits

A significant theoretical emphasis in the pediatric literature over the past four decades has been on explanations of the underlying mechanisms of attentional disorders (Barkley, 1998). In the 1960s, clinicians and researchers emphasized the "hyperactive" or "hyperkinesis" elements of what would eventually be labeled as ADHD (e.g., Chess, 1960). The early 1970s saw a more inclusive focus on the attentional difficulties of children who also had trouble with impulsivity and overactivity, with particular significance attributed to "sustained" attention as a central deficit in this disorder (e.g., Douglas, 1972). Much neuroscience and clinical research has been devoted to investigating the specific operations involved in sustained attention and impulsivity in individuals with "attentional" problems (see the reviews of Corkum \& Siegel, 1993; Parasuraman \& Davies, 1984; van Zomeren \& Brouwer, 1994).

In fact, specific behavioral tasks have even been designed to measure these two processes. Two common examples are reaction time tasks and continuous performance tests (CPTs) (Barkley, 1988; Gordon \& Barkley, 1998). Reaction time activities simply measure the amount of time before responding, following the presentation of a particular stimulus (Douglas \& Peters, 1979). Patterns of errors on these tasks provide important information about the individual's approach to the activity, as in numbers of commissions (responding before the time when a response would be reinforced) or omissions (absence of responding at the appropriate time) (Levy \& Hobbes, 1997). Commissions on these tasks may represent a measure of impulsivity. Continuous performance tasks were developed as a modification to the original 
reaction time tasks. The prototypical CPT activity was developed by Rosvold, Mirsky, Sarason, Bransome, \& Beck (1956). The CPT is assumed to measure an individual's vigilance in attending to a continuous presentation of auditory or visual stimuli, and responding to a particular item or sequence of items. Vigilance in this case can be defined as an individual's ability to sustain attention over a period of time in order to detect infrequent but important stimuli or events (Corkum \& Siegel, 1993). Thus the CPT is reportedly a test of sustained attention. Indeed, many investigators have provided evidence of the utility of reaction time and CPT tasks in discriminating children with attentional difficulties from typically-functioning control children (Halperin et al., 1988; Harper \& Ottinger, 1992; Inoue et al., 1998; Klee \& Garfinkel, 1983; Levy \& Hobbes, 1997; Losier, McGrath, \& Klein, 1996; O'Dougherty, Nuechterlein, \& Drew, 1984; Sykes, Douglas, \& Morganstern, 1973).

However, the constructs of vigilance and sustained attention as purportedly measured on the CPT were apparently not well-understood at first (Corkum \& Siegel, 1993). In fact, several researchers have indicated no compelling evidence of a sustained attention deficit in individuals with ADHD (Corkum \& Siegel, 1993; Loge, Staton, \& Beatty, 1990). This may have reflected an assumption of sustained attention as a unidimensional process. More recent research has suggested multiple dimensions of vigilance and sustained attention; this conceptualization has enhanced the understanding of the roles of these functions for individuals with ADHD. For example, one recent hypothesis about vigilance proposed two separate cognitive processes: arousal (i.e., perceptual sensitivity to a specific event), and response planning and output (van der Meere \& Sergeant, 1988; van Zomeren \& Brouwer, 1994). Signal detection theory has provided a way to distinguish between these two processes in order to measure vigilance (Losier et al., 1996; Pastore \& Scheirer, 1974; Power, 1992). That is, responses on vigilance tasks, such 
as the CPT, can be separated according to the individual's ability to differentiate target from distractor stimuli (perceptual sensitivity), and the subject's "response bias", which reflects the decision-making process for responding or not (Losier et al., 1996; Power, 1992). The importance of measuring sustained attention across time is purportedly to identify decreases in appropriate responding, or vigilance decrements, as described by Parasuraman and Davies (1984). However, in most of the reviewed investigations involving children with ADHD, there was actually stronger evidence for deficits in arousal, as measured by perceptual sensitivity, than for vigilance decrements, as measured by response criterion (Losier et al., 1996; van der Meere, Wekking, \& Sergeant, 1991). Thus, sustained attention appears to be a multi-dimensional cognitive process, of which specific aspects might be more strongly associated with the characteristics of ADHD.

Barkley attempted to address these and other issues in his development of a comprehensive theory about the underlying processing deficits in ADHD (Barkley, 1997, 1998). He cited as the basis of his theoretical framework the work of Sergeant and van der Meere regarding deficits in arousal and motor planning (Sergeant, 1995; Sergeant \& van der Meere, 1988), as well as the work of Quay $(1988,1997)$ and Schachar, Tannock, and Logan (1993) regarding poor response inhibition. Combining these ideas, Barkley proposed that deficits in behavioral inhibition, in particular, are central to ADHD, and that they negatively affect an individual's self-regulatory functions and motor control systems. In this model, four executive functions are thought to be impacted by difficulties with inhibiting prepotent responses, interrupting an ongoing response, and controlling interference factors. Those four functions include: (1) nonverbal working memory (i.e., holding events in memory; anticipating certain outcomes and associations; being aware of appropriate time frames), (2) verbal working memory 
(i.e., "self-talk" about problem-solving; moral reasoning; and rule-governed behavior), (3) selfregulation of affect, motivation, and arousal (includes perspective-taking, and self-monitoring during goal-directed activity), and (4) reconstitution (i.e., analysis and synthesis of behavior; creativity and diversity in goal-directed behavior). Deficits in these functions in turn negatively affect motor responses, such as the actual execution of goal-directed actions, inhibition of irrelevant behaviors, and sensitivity to feedback on behaviors (Barkley, 1997, 1998). The concept most basic to these areas of cognitive deficits is timing, which seems to play a central role in most of the behavioral "errors" committed by the individual with ADHD. Examples include blurting out answers in class (not waiting to be called upon), interrupting others, touching or exploring items immediately upon observing them (not waiting for instructions), rushing through schoolwork assignments, and not being able to delay gratification. Clearly, behavioral disinhibition goes well beyond simple "impulse control".

Within Barkley's proposed theory, sustained attention can be thought of as "goal-directed persistence" (1998, p. 246). That is, the individual's efforts at maintaining arousal to and perception of incoming information is a function of a "decision" to complete a task according to specific rules and required behavioral responses. Thus, arousal and "attentiveness" are considered subordinate functions of behavioral regulation.

Barkley's arguments about behavioral disinhibition are consistent with neuroscience investigations that indicate the right prefrontal and frontal-limbic regions of the brain as being involved in motor-intentional, persistent behavior (Kertesz, Nicholson, Cancelliere, Kassa, \& Black, 1985; Niedermeyer, 1998; Pardo, Fox, \& Raichle, 1991; Whyte, 1992). Further, developmental studies have provided evidence that improved performance on attentional tasks during infancy and toddlerhood is correlated with maturation of frontal cortex functions (Berman 
\& Friedman, 1995; Diamond \& Doar, 1989). Investigation of the role of these functional areas of the brain for individuals with ADHD has been elucidating.

Prefrontal and frontal pathway anomalies, particularly in the right hemisphere, have been identified in children and adolescents with ADHD through a variety of neuropsychological tests purported to assess frontal lobe functioning (e.g., CPT, Wisconsin Card Sorting Test, Stroop Color-Word Test, Rey's Complex Figure) (Carte, Nigg, \& Hinshaw, 1996; Carter, Krener, Chaderjian, Northcutt, \& Wolfe, 1995; Garcia-Sanchez, Estevez-Gonzalez, Suarez-Romero, \& Junque, 1997; Gorenstein, Mammato, \& Sandy, 1989; Grodzinsky \& Diamond, 1992; Korkman \& Peltomaa, 1991; Seidman, Biederman, Faraone, Weber, \& Ouellette, 1997; Shue \& Douglas, 1992; Trommer, Hoeppner, Lorber, \& Armstrong, 1988a; see also the reviews of Barkley, Grodzinsky, \& DuPaul, 1992; Colby, 1991; Faraone \& Biederman, 1998; Niedermeyer, 1998; and Zametkin \& Liotta, 1998).

Although the correlation between neuropsychological measures and specification of neuroanatomical "locations" is not an exact science (Taylor \& Schatschneider, 1992), advances in neurological evaluations have provided further evidence of frontal area problems in persons with ADHD. For example, neurobehavioral studies of visually-evoked potentials during performance of CPT tasks have produced findings of frontal abnormalities in individuals with ADHD (Silberstein et al., 1998; Strandburg et al., 1996). Further, although parietal and temporal lobe anomalies, which are more related to arousal, have also been identified in some persons with ADHD (Filipek et al., 1997; Oades, 1998; Posner \& Petersen, 1990), findings of structural and functional MRIs have indicated more significant involvement of frontal structures and pathways in attentional and inhibition difficulties (Aman, Roberts, \& Pennington, 1998; Castellanos et al., 1994; Giedd et al., 1994; Filipek et al., 1997; Hynd, Hern, Voeller, \& 
Marshall, 1991; Oades, 1998; Rogeness, Javors, \& Pliszka, 1992; Swanson, Castellanos, Murias, LaHoste, \& Kennedy, 1998). In developmental studies of brain maturation, there is also evidence that ADHD characteristics may become more salient as certain frontal structures (e.g., caudate nucleus) grow and mature (Mataro, Garcia-Sanchez, Junque, Estevez-Gonzalez, \& Pujol, 1997; Oades, 1998). Thus, there appears to be stronger evidence in ADHD for the involvement of brain structures that are related to behavioral inhibition than to thalamic, midbrain, and parietal structures that are related to arousal and orienting (Aman et al., 1998; Colby, 1991; Denckla, 1996; Filipek et al., 1997; Shue \& Douglas, 1992). These latter structures are obviously important in the integrated processes that produce "attending", by first stimulating arousal mechanisms. However, the motor-intentional functions of the frontal structures and pathways seem to be more involved in ADHD (Niedermeyer, 1998).

Denckla (1996) made remarkably similar points about the central deficits in ADHD, by suggesting difficulties of intention, in which individuals demonstrate a poor ability to inhibit responding. She further suggested that those problems with disinhibition affect learning and memory in children with ADHD, which is consistent with the ample documentation of the cooccurrence of learning disabilities in children with attentional deficits. In fact, researchers are just beginning to sort out the various processing and performance deficits in "pure" groups of ADHD children, versus learning disorders that are also characterized by symptoms of attentional difficulties (Kataria, Hall, Wong, \& Keys, 1992; Korkman \& Pesonen, 1994; Nigg, Hinshaw, Carte, \&Treuting, 1998). This quandary is addressed in more detail in the following section. $\underline{\text { Relation between attention and learning }}$

First, a definition of "learning disabilities", as related to this paper, must be undertaken. Many researchers and clinicians use a discrepancy model to operationally define learning 
disabilities, by documenting a specific difference (usually based on standard deviations of a standardized score) between assessed intellectual aptitude, and achievement in particular academic areas (Aylward, Verhulst, \& Bell, 1990; Lambert \& Sandoval, 1980). This strategy is, in fact, described in the DSM-IV (APA, 1994) as the criteria for diagnosis of Specific Learning Disorders (categorized according to reading, arithmetic, written expression, and "other" specific disorders). Barkley recognized, however, that determining the criteria for the discrepancy between achievement and cognitive functioning is arbitrary (e.g., achievement scores 1.5 or 2 standard deviations below IQ scores, or achievement scores below the $25^{\text {th }}, 10^{\text {th }}$, or even $7^{\text {th }}$ percentile, compared to an average IQ score), and that various models can result in more (or less) rigorous classification of individuals with learning disabilities (Barkley, DuPaul, \& McMurray, 1990).

An alternative definitional model is often used by neuropsychologists, who tend to regard learning disorders in a broader sense (Denckla, 1996). Neuropsychological testing evaluates the information-processing skills and associated brain structures that appear to be related to the overt "behaviors" of academic deficits. This theoretical model of learning deficits can take into account other types of discrepancy profiles, such as a significant deficit in overall functioning in one area of cognitive problem-solving versus "normal" aptitude in another domain area (e.g., the example of a nonverbal learning disability, with average verbal and reading abilities and significantly poorer scores on Performance IQ measures and nonverbal academic tasks, as described by Rourke and colleagues (Harnadek \& Rourke, 1994; Rourke, 1989). Thus, it seems important to consider both of these conceptualizations of cognitive/learning discrepancies when defining "learning disabilities", particularly as related to the ADHD population. For purposes of the present paper then, both the intellectual ability/achievement discrepancy (using the generally- 
accepted criterion of achievement scores two or more standard deviations below IQ score) and the performance/verbal learning discrepancy models were used to identify children with ADHD as having comorbid learning disabilities.

As Denckla (1996) noted, this broader definition of learning disabilities allows for consideration of the learning process, which is likely the best way to think about the learning difficulties of individuals with ADHD. She suggested that for many persons with ADHD, an additional manifestation of problems, beyond behavioral disinhibition, is in learning and memory deficits. The four generally-accepted processes of learning and memory include encoding or acquisition, consolidation, storage, and retrieval (Schneider \& Pressley, 1989). Scientific and clinical evidence suggests that children with ADHD demonstrate difficulties primarily with the initial stage, due to their poor application of learning strategies such as organization and rehearsal (Barkley, 1997, 1998; Barkley et al., 1992). The implication is that if the information is initially perceived and encoded appropriately, consolidation and storage can proceed effectively (Kaplan, Dewey, Crawford, \& Fisher, 1998). Research has indeed found that children with ADHD demonstrate limited time allowances for observing/listening to presented information, and poor strategies for manipulating and organizing that material $\left(\mathrm{O}^{\prime} \mathrm{Neill} \&\right.$ Douglas, 1996; Sonuga-Barke, Taylor, \& Heptinstall, 1992). This fits with a theory of behavioral disinhibition, in that the child has trouble delaying his responses, and too quickly acts on information without taking important steps to make the material more meaningful and useful. Thus the child with ADHD has trouble learning new information. It also follows that ADHD children may experience inconsistencies with recall of information, due to those impulsive and disorganized approaches to tasks. Denckla (1996) referred to this behavior as a "producing disability" (p.117). 
It is interesting to note, however, that children with ADHD appear to display adequate incidental social learning, as well as memory for routines and frequently-repeated information and activities. Thus, the executive function that defines rapid memorization skills (e.g., mnemonic strategies, and episodic, rote memory), which is more frequently required for learning in academic settings, appears to be impaired in individuals with ADHD. However, more vicarious learning, which occurs through observation, imitation, and practiced routines, appears to be unaffected in most ADHD children (Denckla, 1996). Indeed, deficits in long-term memory appear to be uncommon for individuals with ADHD (Kaplan et al., 1998). These observations lend further support to the notion of disordered acquisition and encoding processes versus consolidation and storage processes in learning and recall for persons with ADHD.

Both Denckla (1996) and Barkley $(1997,1998)$ discussed how these difficulties in attentional, encoding, and decision-making processes are related to working memory deficits in individuals with ADHD. It is during the early stages of learning that the individual must "work with" the incoming information, in order to begin to consolidate it for storage and eventual retrieval. This "working time" requires some delay between presentation of a stimulus and the person's response, which is a characteristic problem for individuals with ADHD. Indeed, conceptualization of working memory as a bridge between attending to and acting upon incoming information suggests a learning component to this function (Baddeley, 1986). This fits with Denckla's notions about deficits in executive function for individuals with learning disabilities, and Barkley's hypothesis regarding deficits in verbal and nonverbal working memory for individuals with ADHD. Verbal working memory difficulties, for example, which would include problems in developing "rule-governed behavior", could potentially be accounted for by verbal learning disabilities. Nonverbal working memory difficulties could be what is manifested 
in performance difficulties such as rapid but inaccurate motor responses (e.g., raising hand immediately after a teacher's question, without really having an answer; or rapidly producing written products that are functionally illegible). Indeed, documentation exists for reading and language disabilities associated with ADHD (August \& Garfinkel, 1990; Korkman \& Pesonen, 1994; McGee, Williams, Moffitt, \& Anderson, 1989; Nigg et al., 1998; Tirosh \& Cohen, 1998), and for performance difficulties such as written language problems (Harnadek \& Rourke, 1994). Neuroanatomical evidence can again be cited in support of these ideas about working memory, in that findings have shown that children with ADHD and comorbid learning disorders demonstrate more difficulties on tests of frontal systems than individuals with ADHD and no diagnosable learning difficulties. This suggests connections between centers of processing and executive functions (Herschkowitz, Kagan, \& Zilles, 1997; Lazar \& Frank, 1998; Niedermeyer, 1998; Wolfe, 1996). Taken together, these findings point to a significant relation between behavioral disinhibition and working memory functions.

In addition to working memory, the study of other types of memory (e.g., short-term, long-term) provides another avenue for understanding attention and its impact on cognitive functions. Just as developmental aspects are important in thinking about attentional functioning in children and adolescents, maturational differences in memory are critical to a discussion of learning and performance. The next section further addresses theories of memory development, and the difficulties that attention deficits may pose when an individual tries to remember and recall information.

\section{Relation between attention and memory}

The behavior analytic conceptualization of attention as a function of stimulus control, described earlier in this paper, can be extended to memory. Branch (1977) suggested that 
memory for a particular stimulus-response relation depends upon the strength of the association between the two factors; this association is developed, at least in part, via the individual's attention (measured in responses) to the stimulus. In another line of study, neuropsychological tests have provided evidence that the same brain structures that are associated with organization and inhibitory aspects of functioning (i.e., the frontal lobe and the prefrontal-limbic system connection; Barkley et al., 1992) are also known to be involved with immediate and working memory abilities (Welsh \& Pennington, 1988). Thus, measurement of memory in children with attentional difficulties might contribute to a better understanding of the specific aspects and functions of attention (Adams, Robins, Sheslow, \& Wilkinson, 1992). First however, theories of memory development should be briefly discussed.

Conceptualizations of Memory. Current theories of remembering and forgetting have ties to the work of Ebbinghaus in Germany, in the late 1800s. Much of that early research emphasized the span of memory capacity, in terms of number of items remembered in a sequence of stimulus presentations (cited in Schneider \& Pressley, 1989). William James (1890, in Sheslow \& Adams, 1990) wrote about primary and secondary memory, which is related to current conceptualizations of short- and long-term memory. Other work in the area of memory around the turn-of-the-century involved (a) a description of learning curves, which focused on increases in responding as a function of repeated exposures to particular set of stimuli; and (b) primary and recency effects in remembering, in which the initial and final parts of presented information are recalled more frequently than the medial portions of stimuli (as in word lists) (Sheslow \& Adams, 1990).

Formal theorizing about memory in the American psychological literature began with hypotheses regarding stimulus-response relations and habit strength (Hull, 1943; Watson, 1924; 
both cited in Hothersall, 1990). Habit strength was defined as a reflection of the degree of association between a stimulus and a response. If the association was strong, and the same response was consistently emitted in the presence of a given (discriminative) stimulus, the individual was said to have "remembered" the association. This theory did not include an internal, mediational mechanism for memory storage or retrieval. It is strikingly similar to current operant conceptualizations of memory, as described above (Branch, 1977). These types of theories have frequently been criticized, however, for a lack of comprehensiveness in addressing all of the functional aspects of memory (Sheslow \& Adams, 1990; Schneider \& Pressley, 1989).

Many of the subsequent theoretical formulations about memory have been based on information-processing models of learning and remembering, most of which have some roots in computer analogies of processing systems. Information processing models conceive of learning, memory, and recall as a dynamic process that involves a generally predictable chain of interconnected steps (Boyd, 1988). That is, information presented to an individual can be perceived as moving through an anticipated series of "events" much like data that is input to a computer: the stimuli are initially "sensed", and then interpreted and saved (if the appropriate work is undertaken to accomplish these steps) so that retrieval at a later time, as prompted by a similar set of stimuli, can rapidly occur. In the computer, these steps are prompted by certain commands; in the brain, these steps are prompted via neurotransmitters and synaptic connections (Squire, 1987).

The predominant information-processing models for memory development include structural, "multi-store" components (Atkinson \& Shiffrin, 1968), and hierarchical levels of processing (Craik \& Lockhart, 1972). The former model posits that information is first 
registered as a literal sensory copy of the incoming information, then, with minimal rehearsal, the information can be stored in and recalled from a short-term storage "area." Given that this "area" has only limited storage capacity, the information, if continuously or frequently rehearsed, is moved relatively quickly into a longer-term store with unlimited capacity (Atkinson \& Shiffrin, 1968; Boyd, 1988). The sensory register stage can be said to involve encoding of incoming information, while short-term memory represents the beginnings of consolidation processes. Further consolidation, as well as storage and retrieval, are assumed to be functions of long-term memory (Sroufe et al., 1996). In this model, attentional skills are thought to be involved in the perception of stimuli that are registered sensorily, and in the transfer of that information from the sensory register to the short-term memory area. Attention in this case is sometimes referred to as "primary" memory (Fastenau et al., 1998); these processes seem to be very akin to current conceptualizations of working memory.

The levels of processing theory suggests that information that is processed (encoded) at a cursory or sensory level will be able to be recalled for only a short time, and then quickly forgotten. Information that is processed more "deeply" (consolidated), in terms of patterns and contextual meaningfulness, will be retained (stored) and accessible for retrieval for longer periods of time (Boyd, 1988; Craik \& Lockhart, 1972). This view seems to have more developmental implications than the multi-store model of memory functioning. For example, levels of processing implies that memory development in children improves as their capacity to attend to (i.e., perceive, encode, consolidate) more stimulus cues increases. That is, as young children develop greater ability for acquiring and storing more pieces of information, identifying salient and relevant features of the learning environment, and identifying relations among pieces of information through organization and categorization, they become better able to process 
information at "deeper" levels (Boller \& Rovee-Collier, 1992; Cohen \& Younger, 1984; Halford et al., 1994; Hartshorn et al., 1998; Kail, 1990). In this model then, developmental progress in a variety of attentional and learning functions is related to improved memory ability.

Baddeley (1986) considered the levels of processing theory to be closer to a conceptualization of a working memory model. Active processing of information, to form a durable memory, was similar to Baddeley's ideas of mentally manipulating information. However, he recognized that the levels theory, and the subsequent research conducted to support it, emphasized long-term memory. His own development of a working memory model was reportedly spurred by the limited formulations about short-term memory in the two "traditional" models of memory functioning described above.

Despite the developmental implications of the levels of processing theory, both it and the multi-store models of memory development were actually developed and investigated with an emphasis on adult memory (e.g., Lashley, 1929, reviewed in Boyd, 1988; Sheslow \& Adams, 1990)). That emphasis strengthened following World War II, given the increase in neuropsychological studies of soldiers with head injuries. Results revealed significant and specific disruptions in remembering as a result of focal brain lesions (e.g., Scoville \& Milner, 1957). Overall however, information regarding adult memory was problematic for interpreting memory abilities in children, in that considerations of changes in neuroanatomical structures and cognitive functioning at different stages of development were not addressed. Developmental theorists initially attempted to fit the existing models to maturational changes in cognitive and memory abilities, but eventually devised more specific frameworks for studying memory in children (Brown, 1975; Jenkins, 1979). 
Developmental aspects of memory. Jenkins' (1979) tetrahedral (four-factor) model

involved two factors related to task characteristics: the nature of the information to be learned, and the task paradigms for acquiring and retrieving the material. The other two factors focused on characteristics of the child (e.g., cognitive abilities, skills, knowledge, and motivation level), and on activities that the child must demonstrate in order to acquire and remember information (e.g., orienting and attending to the stimulus presentation, understanding task instructions, recognizing familiar information, etc.). Ann Brown and her colleagues (Brown, Bransford, Ferrara, \& Campione, 1983) emphasized that developmental progress, primarily among learner characteristics in the model, are associated with improvements in memory functioning. Examples of such progress would include expansion of the child's knowledge base (linked with experiences), greater efficiency of cognitive processing, and implementation of organizational strategies such as rehearsal and mnemonics. This reasoning suggests that processing "capacity" per se does not increase, as suggested by some researchers (Pascual-Leone, 1970; Burtis, 1982), but rather that developmental maturation accounts for improved memory functioning over time (Halford et al., 1994). Within this model then, attentional and learning differences could account for significant sources of variance in relation to learner characteristics.

Brown's (1975) own model of memory development in children took into account potential improvements in the learner's cognitive abilities, as described above. She suggested a different framework though, to include three continua, each of which addressed developmental readiness for implementing strategies of remembering. The continua included (a) the need for strategy versus no strategy in recalling information, (b) the ability to implement a mediational strategy spontaneously versus being taught to purposefully produce it, and (c) the ability to recall episodic (discrete, relatively meaningless) information versus semantic (contextually-based, 
meaningful) material. The performance of the child would indicate where he or she functions developmentally, in relation to each of the elements of remembering. Two types of developmental changes can be detected within this model: (a) quantitative improvements in skills across age and maturation, and (b) qualitative improvements in skills in terms of level and patterns of difficulty. Evaluation of an individual's range of development for each of the memory tasks might provide information about any problems with remembering information. For example, Barkley (1998) and Denckla (1996) suggested that children with attention deficits often demonstrate difficulties in spontaneously employing mediation strategies on learning tasks, which can result in "producing" disabilities. Other investigators have found that children with ADHD demonstrate deficient use of rehearsal strategies in attempting to learn and recall new information (O'Neill \& Douglas, 1991; 1996). The boys in the latter studies did not discount that rehearsal strategies were helpful, but rather they demonstrated inefficient application of those strategies to perform recall tasks.

In considering these models of developmental changes in memory, the question arises as to when the developmental process is "complete" and memory functioning is more stable and similar to an adult's skills (albeit a young to middle-aged adult, recognizing that older adults often experience gradual declines in memory abilities). Gathercole (1998) suggested that memory function undergoes significant, qualitative changes in development through age 7 years; then an individual's skills for organizing information, and implementing rehearsal and mediation strategies, are similar to that of an adult. She did acknowledge that more subtle quantitative improvements in memory functioning occur during older childhood and adolescence. An example of those types of improvements is the finding that adolescents aged 16-19 years performed more effectively and efficiently on tasks of short-term memory which included an 
intervening distractor task, than younger adolescents aged 12-14 years (Ryan, 1990). Other

investigations have found similar age-related competencies in visual-spatial and verbal working memory (e.g., Swanson, 1996) and in use of mnemonic strategies for information retrieval (Ackerman, 1996; Hashimoto, 1991). These findings have significant implications for the development of tests of memory for children and adolescents, which will be discussed in the next section.

A related question in the developmental literature about memory is whether improved competencies for remembering involve a continuous or discontinuous process. Across those early learning years, does one new set of competencies lead to another by building on what has already occurred, or is there a discontinuous progression of qualitative changes that characterize development? The work of both Jean Piaget (Ginsburg \& Opper, 1969; Pascual-Leone, 1970) and Jerome Kagan (1979) supported a discontinuous model of development, wherein significant qualitative changes, such as differences in cognitive operations and emergence of language, are obviously important developmental competencies but do not hold a specific link to the history of early infancy. However, a number of more recent research studies have provided support for a theory of continuity in development, particularly in learning to encode and recall information (Bauer, Hertsgaard, \& Dow, 1994; Bornstein \& Sigman, 1986; Boyer, Barron, \& Farrar, 1994; Howe \& Courage, 1997; Myers, Perris, \& Speaker, 1994). One example thought to reflect continuity and integration among cognitive processes is the finding of strong correlations between efficiency of visual and/or auditory recall in infancy, and performance on standardized tests of intelligence, achievement, and language in middle childhood (Bornstein \& Sigman, 1986; Rose \& Feldman, 1997). Another potential example is the increasing use of verbal 
mediation in solving visual-spatial tasks as children get older, suggesting the continuous development and gradual integration of skills across modalities (Fastenau et al., 1998).

Taken together, this evidence for maturational changes in memory development implies that assessment of children's learning and recall should be different from adult-oriented evaluation procedures. That is, tests of memory functioning must be developmentally sensitive to those maturational changes. This topic is addressed in the next section, along with assessment issues related to attentional functioning.

Implications of the relations between attention, learning, and memory

The theoretical information reviewed thus far provides a framework for understanding the current conceptualization of the underlying deficits in ADHD, and the relation of those cognitive and behavioral difficulties to learning and memory. Problems with sustained attention and impulsivity, "traditionally" thought to be the primary difficulties in ADHD, are now believed to be subsumed under a more pervasive and multidimensional deficit in regulation of behavioral inhibition. This disinhibition problem has far-reaching effects related to a variety of cognitive functions, including learning, working memory, and longer-term recall. In considering the behavioral and performance manifestations of those cognitive difficulties, one question that arises is how to measure each of those processes. A related question would be whether different profiles obtained on a battery of attentional, learning, and memory tests would be representative of different clinical diagnoses. For example, do children with ADHD and no diagnosable learning disorders perform differently on the types of tests noted, versus children with comorbid ADHD and LD? Further, what would the assessment profiles of children with other conditions that have associated attentional and memory problems, such as TBI, look like? In the following section, two commonly used assessment tools in the measurement of attentional functioning, and 
learning and memory skills, respectively, are reviewed, in terms of both validity characteristics and application to the three clinical groups mentioned above (e.g., children with ADHD, LD + ADHD, TBI). This discussion will lead to the statement of the purpose of the present study. Assessment of attentional behaviors. As described previously, instruments designed to evaluate sustained attention and inhibition of impulsive or overlearned responses have been increasingly utilized as part of a battery of tests to assess and classify individuals with ADHD (e.g., McMillan, Walters, \& Holder, 1993). Activities such as reaction time tasks and CPTs were developed to measure the relation between cognitive strategies and overt behavioral responses (Douglas, 1983). Gordon (1983) developed a version of both types of tasks, housed in one portable electronic system. The Gordon Diagnostic System (GDS) is one of the most commonly used tools in the evaluation and identification of children with ADHD.

Gordon Diagnostic System (GDS). The two original tasks of the GDS were designed to measure controlled inhibition of behavioral responses, and what was thought to be sustained attention, respectively, in children with ADHD (Gordon, 1983; Gordon \& Mettelman, 1988). The Delay task was developed as a modified version of the reaction time task, and is assumed to measure impulsivity. It is based on the notion of differential reinforcement of low rates of behavior, from applied behavior analysis work (Gleeson, 1991). The child is required to pace his responding, in order to receive reinforcement (points) for responses emitted after a set interval of time. Efficiency of responding is the primary measure obtained for this activity. The Vigilance task was designed to be similar to continuous performance tests. It measures the child's ability to detect a particular stimulus set, consisting of two specific numerals (1 and 9) presented sequentially, within a continuous presentation of randomly-presented, single-digit numerals on an electronic screen. Measures of performance include number of correct responses, as well as 
patterns of omission and commission errors. A third "subtest", the Distractibility task, was

added to the battery later, and involves the same response requirements as the Vigilance task.

However, a higher rate of distractor stimuli is included, in order to measure potential interference with attentional capabilities.

The GDS is one of the few reaction time/CPT measures for which standardization data have been collected and published (see detailed information in Procedures section below; Gordon \& Barkley, 1998). The clinical efficacy of the GDS in discriminating elementary-aged children with ADHD from normally-functioning individuals has been documented in many investigations (Barkley, 1991; Barkley et al., 1990; Breen, 1989; DiNiro, 1987, cited in Gordon \& Mettelman, 1987; Gordon, 1979, 1986; Gordon, DiNiro, \& Mettelman, 1988; Grodzinsky \& Diamond, 1992; McClure \& Gordon, 1984; Oppenheimer, 1986, cited in Gordon \& Mettelman, 1987). This assessment instrument has also been shown to be sensitive to the effects of psychostimulant medication in elementary-aged children with attention deficits and overactivity, but more so at moderate to high, rather than low, doses (Barkley, DuPaul, \& McMurray, 1991; Barkley, Fischer, Newby, \& Breen, 1988; Cohen, Kelly, \& Atkinson, 1989; Fischer \& Newby, 1991; Fischer, 1996; Rapport, DuPaul, Stoner, \& Jones, 1986; Rapport et al., 1987). Hall and Kataria (1992) found that the Delay, but not the Vigilance, task of the GDS was sensitive to the combined effects of psychostimulant medication and a cognitive training program about how to perform the GDS tasks, in 6-12 year old children.

Although the Delay task was, in fact, suggested as the most significant discriminating factor on the GDS in several early validation studies (Barkley, 1991; Breen, 1989; Gordon, 1979; McClure \& Gordon, 1984), these findings were not supported in more recent investigations that compared the GDS to other measures of attentional, behavioral, and cognitive functioning. 
Barkley and his colleagues (Barkley et al., 1988) suggested that perhaps the GDS Delay task does not actually measure the type or nature of impulsive behavior, or behavioral disinhibition problems, that children with ADHD demonstrate. Further, given Barkley's new conceptualization of ADHD (1997, 1998), perhaps the reason that the GDS Delay task seems to have poor discriminative validity for this population is that impulsivity, as traditionally defined, is not really the "hallmark" deficit in ADHD.

Instead, many investigations have identified the Vigilance task (and the Distractibility task when administered) as contributing more of the variance in discriminative classification of children with ADHD (Breen, 1989; Cohen et al., 1989; Grant, Ilai, Nussbaum, \& Bigler, 1990; Loge et al., 1990). Although sustained attention is subsumed under the pervasive behavioral disinhibition problem in Barkley's theory, it may be one valid measure of that deficit.

Correlations between behavior rating scales, the most commonly used tools in the diagnosis of ADHD (Conners \& Barkley, 1985; Schaughency \& Rothlind, 1991), and GDS scores provide an example. Total Correct scores from the GDS Vigilance and Distractibility Tasks have been found to correlate positively with the ADHD Rating Scale (Wherry et al., 1993), the Child Behavior Checklist (Gordon et al., 1988; Gordon, DiNiro, Mettelman, \& Tallmadge, 1989), the ADD-H Comprehensive Teacher Rating Scale (ACTeRS) (Cohen et al., 1989; Gordon et al., 1988), and the Conners' Parent Rating Scale - Revised (Cohen et al., 1989). In all of those studies, however, teacher ratings of elementary students' behaviors correlated more significantly with the GDS scores than did parent ratings. This is an interesting finding, in terms of both situational variables related to assessment, and the assumptions of what each type of evaluation strategy is measuring. As Conners (1998) noted, behavior rating scales are subject to such biases as contributing environmental variables (e.g., clinical vs. school vs. home settings, comparisons 
to others/peers present in the setting, recency of behavioral episodes), and the characteristics and familiarity of the person answering the questions. Even in a more objective testing situation with an instrument like the GDS though, environmental variables can still play a critical role in performance (Power, 1992). For example, degree of novelty in a clinical setting, as well as examiner presence or absence during testing, have been found to affect children's performance on CPT measures (Barkley, 1998; Power, 1992; Schaughency \& Rothlind, 1991). Further, it may be that the GDS Vigilance and Distractibility tasks measure behaviors that are correlated more with school-related demands for attention and behavioral inhibition than with broader impressions of attention and behavior identified by parents. To address these concerns when conducting an assessment for ADHD, many investigators have suggested the importance of using report measures such as rating scales in conjunction with quantitative measures such as the GDS. Observation of behavior during the performance measures is also imperative, taking into account novelty factors as well as the child's relationship with the examiner, his demonstrated level of motivation, and his variability in performance and response times across the tasks (Barkley et al., 1990; Gordon et al., 1988; Gordon et al., 1989; Grant et al., 1990; Trommer, Hoeppner, Lorber, \& Armstrong, 1988b).

Concurrent validity of the GDS with measures of cognitive and achievement ability has also been investigated. A number of studies with elementary-aged children have found that Total Correct scores on the Vigilance and Distractibility tasks correlated significantly with the subtests that comprised a Freedom from Distractibility Factor on the WISC-R (Arithmetic, Digit Span, and Coding; Kaufman, 1979), arithmetic subtests on achievement measures, some sensorymotor measures (tactile and auditory), and some higher-order cognitive processing measures (e.g., Raven's Progressive Matrices, Category Test) (Grant et al., 1990; Loge et al., 1990; 
Trommer et al., 1988a). The GDS Delay Task has less consistently been found to be related to cognitive measures of attention. In the Grant et al. (1990) study, for example, scores on the Delay task were significantly correlated only with the arithmetic subtest of an achievement measure, and with a few of the nonverbal problem-solving measures (e.g., Beery Test of Visual Motor Integration, Tactual Performance Test).

The finding that the GDS Vigilance and Distractibility tasks were correlated with some higher-order cognitive processing skills led to the suggestion that those continuous performance tests may in fact measure more than simply sustained attention (Grant et al., 1990; Trommer et al., 1988a). This assertion begs the question of the interaction between attention, intelligence, and reasoning and problem-solving abilities. That is, are measures of sustained attention impacted by complex processing abilities, and/or is performance on intelligence and cognitive tests related to level and maintenance of attention (Barkley, 1988; Sattler, 1992; Trommer, Lorber, Armstrong, \& Hoeppner, 1987)? Although intelligence scores on the WISC-R and Slosson Intelligence Test did not contribute significantly to the variance in performance among the GDS standardization sample (Gordon \& Mettelman, 1988), findings have been different among clinically-referred samples. Trommer's and Grant's groups did find some significant correlations between the GDS Vigilance and Distractibility tasks, and auditorally-presented arithmetic subtests and some visual-spatial subtests of the WISC-R, as noted above. Further, moderate correlations were found between performance on all indices of the Kaufman Assessment Battery for Children (K-ABC) and scores on the Vigilance Task of the GDS (Gordon, Thomason, \& Cooper, 1990). Loge's group, however, did not find support for correlations between performance on the GDS and on cognitive-processing activities such as the Wisconsin Card Sorting Test and verbal and design fluency tasks. They agreed, though, that 
difficulties in directing and maintaining attention to task represented a significant deficit for their elementary-aged ADHD subjects (Loge et al., 1990). Taken together, these findings suggest that attention is a central mechanism of intelligence and cognitive functioning, and contributes to performance on tests of cognitive and academic skills. Thus, it may a prudent suggestion to consider assessment of attentional/behavioral and intellectual/achievement abilities in most children referred for a question of ADHD or related difficulties, in order to obtain appropriate diagnostic and treatment information.

It is essential to note at this point that some of the discrepancies apparent in the reports of discriminative and concurrent validity of the GDS may be related to two confusing issues in the reporting of GDS data. First, many investigators used only the "abnormal" scores under the GDS classification system in reporting results of validation studies (Cohen et al., 1989; Trommer et al., 1988b). That system reflects normality versus abnormality, based on percentile ranks obtained with the standardization sample. For each of the data categories, scores are divided into descriptive ranges based on conventional statistical and clinical judgments (Gordon \& Mettelman, 1988). "Normal" scores fall at the 26th percentile or above, based on age-level results of the standardization sample. The "borderline" range represents scores in the 6th to 25 th percentile, and "abnormal" scores fall at the 5th percentile or below. These norms have been combined across gender, given no findings of significant gender effects on performance (approximately 2\% of the variance) (Breen, 1989; Gordon \& Mettelman, 1988).

Cohen and his colleagues (1989) found that including the "borderline" scores of subjects, in addition to the "abnormal" scores, significantly increased the correlations between rating scales and the GDS scores in classifying subjects as having an attentional disorder. This practice might seem to be appropriate in clinical or educational situations, given that most standardized 
psychoeducational measures consider performance below the $30^{\text {th }}$ percentile as "below average"; that cutoff is two standard deviations below the $50^{\text {th }}$ percentile mid-point. Utilizing only the lower $5 \%$ of scores on the GDS may reduce the comparability of those scores to other measures; however, staying within that lower criterion would enhance the sensitivity and specificity of the GDS for detecting "true positives" (regarding an ADHD diagnosis) (K. Hamsher, personal communication, November 1, 1999). Trommer et al. (1988b) suggested avoiding those issues in research analyses, by using absolute numerical scores from the GDS. This strategy could provide increased specificity about GDS performance, as well as improved intercorrelations with cognitive and behavioral comparison measures. Thus, research investigations that report only the descriptive categories for GDS scores may produce findings that are questionably generalizable, and difficult to interpret.

Another significant factor which likely has affected the interpretation of reported GDS results is the heterogeneity of subject groups in terms of comorbid learning difficulties. Many of the studies reported that their ADHD samples included a number of individuals with an additional diagnosis of a learning disorder. Cohen et al. (1989), for example, indicated that a third of their sample had been identified as having comorbid learning disabilities. Several other studies mentioned the co-existence of LDs in their subject pools but gave limited specifications (e.g., Loge et al., 1990), and even more did not address the presence of learning difficulties at all (e.g., Trommer et al., 1988a; Wherry et al., 1993). However, even those studies that mentioned concurrent learning problems did not sort out results according to the differences in subject characteristics.

As described earlier, the prevalence of LDs in children with ADHD can be quite significant, and thus it is important to be able to identify, to the extent possible, the relative 
contributions of attentional versus learning problems on performance. CPTs and other measures of frontal lobe functioning have actually been increasingly used in the evaluation of LD in school-aged children, with most findings pointing to Total Correct or Omission rates as being a discriminative factor for LD versus normally-functioning subjects (Brown \& Wynne, 1984; Eliason \& Richman, 1987; Lazar \& Frank, 1998; Levy \& Hobbes, 1989; Richards, Samuels, Turnure, \& Ysseldyke, 1990; Swanson, 1983). Use of the GDS to discriminate between ADHD and LD subjects has yielded positive findings, but more often based on Total Commission scores for the Vigilance and Distractibility tasks than for Total Correct (Barkley et al., 1990; Gordon \& McClure, 1983; Robins, 1992). However, Aylward and his colleagues did not replicate such findings (Aylward et al., 1990). In that study, there was no significant main effect for learning disabilities on the GDS, although the Vigilance task scores did differentiate subjects with attentional deficits from normal controls. However, the subject group in Aylward et al.'s study was young (mean age $=8.4$ years), suggesting that age may be an important covariate to consider. The authors cited Swanson's (1983) finding that an LD effect did become evident in subjects above the age of 10 years. Taken together then, these findings indicate the need to take IQ, achievement levels, and chronological age into account when interpreting the results of continuous performance tests such as the GDS, given the inconsistent findings among investigations that did not control for these variables.

In regard to TBI populations, only one study was identified that evaluated the utility of the GDS in differentiating the attentional capabilities of individuals with traumatic brain injury (TBI) from normally functioning controls (Burg, Burright, \& Donovick, 1995). The subjects in this investigation, however, were adults, with a mean age of 40-41 years. IQ was controlled, and scores on the Vigilance and Distractibility Tasks were found to discriminate the TBI group from 
the controls. Further, low to moderate correlations were found between the Vigilance and Distractibility Total Correct measures, and other neuropsychological measures of attention such as the WAIS-R Digit Span and Arithmetic subtests and the Stroop Color-Word Interference Task. Again, performance on the Delay Task contributed no significant variance to the discriminative analysis. No studies were identified that addressed the GDS' ability to discriminate TBI patients from individuals with ADHD.

In children with a history of TBI, deficits in sustained attention have been documented on other continuous performance tests, particularly in groups of more severely injured individuals (e.g., Kaufmann et al., 1993). Overall omission and commission errors have been reported as the distinguishing measures, versus decrements in vigilance and responses over time. The developmental perspective of attentional functioning is particularly important for this population: recent studies have found no evidence for "preferential sparing", or at least amenability to full recovery of sustained attentional processes, in younger children (e.g., below 10 years of age), as originally thought (Anderson et al., 1997; Kaufmann et al., 1993)

Finally, no studies were identified that specifically addressed the relation between attentional functioning on the GDS and overall memory skills. However, several investigations have indicated correlations between some measures of short-term memory and performance on the GDS subtests (e.g., Grant et al., 1990; Loge et al., 1990; Robins, 1992), suggesting that those types of tasks (e.g., Digit Span subtest on the Wechsler tests) probably measure attention to a significant degree. Verbal learning was also measured in one study (Loge et al., 1990) and was found to be a relative weakness for ADHD subjects compared to control subjects. Otherwise, no specific investigations of the relation between attention and memory functioning were identified. 
Assessment of learning and memory. In his comprehensive chapter on memory in children, Boyd (1988) provided guidelines and suggestions for creating an appropriate assessment tool to measure children's memory functioning. His recommendations were based on the current understanding of developmental changes in memory abilities. Generally, Boyd suggested that a test of memory for children should emphasize strategy use, recall of multiversus unimodal information, and the role of attention in memory functioning. In fact, he recommended that measurement of attentional functioning always occur before memory testing is undertaken. Specific examples of recommended tasks for a developmental test of memory included variations of the four components of memory outlined by Brown (1975): combining strategic versus nonstrategic retrieval processes with semantic and episodic information, respectively. Memory tasks requiring few strategies (e.g., no mnemonics) are thought to measure incidental memory and basic memory capacity. Copying familiar geometric designs, in novel sequences and combinations, is an example of nonstrategic-episodic recall; identifying differences in details between two nearly identical pictures is an example of nonstrategicsemantic recall. Strategic-episodic memory tasks would include multiple-trial learning of word lists, as well as memory span activities such as repeating series of numbers, letters, or hand movements. Strategic-semantic tasks measure the encoding of contextual information; examples would include repeating sentences and recalling short stories.

Sheslow and Adams (1990) attempted to follow many of these suggestions in their development of the Wide Range Assessment of Memory and Learning (WRAML), which is a comprehensive instrument for addressing memory functions in children.

Wide Range Assessment of Memory and Learning (WRAML). The WRAML test construction reportedly involved five major emphases (Sheslow \& Adams, 1990). First, an effort 
was made to distinguish between memory and learning. Each memory subtest involves the presentation of discrete information that must be recalled immediately, and for some subtests, at a later time as well. In contrast, learning subtests purportedly measure both acquisition and recall of information over multiple trials. Second, performance is measured according to modality of presentation of the information: visual versus verbal stimuli, and cross-modal combinations of the two. Third, subtests are varied along a continuum of meaningfulness, analogous to Brown's (1975) episodic-semantic continuum, such that tasks range from presentations of minimally meaningful, abstract material, to contextually-based, concrete information. Subtests also represent the various combinations of strategic and nonstrategic tasks, paired with episodic versus semantic information (as suggested by Boyd, 1988). Fourth, various criteria are involved in the recall of information, including immediate, delayed, and recognition (forced-choice) recall. The span of time prior to presentation of delay and recognition tasks varies from approximately 10 minutes to 40 minutes. Finally, the test was constructed to be able to evaluate children's memory across a wide age range, with a scaled scoring system that allows comparisons of performances across ages and developmental levels.

The nine subtests of the WRAML were organized along three scales, or indices, according to a principal components analysis with varimax rotation of the intercorrelations between subtests (Sheslow \& Adams, 1990). The three indices separately emphasize visual memory, verbal memory, and learning; each scale includes three subtests. The Visual Memory scale includes the subtests of Picture Memory, Design Memory, and Finger Windows. The Verbal Memory scale includes Story Memory, Sentence Memory, and Number/Letter Memory. The Learning scale involves one verbal task (Verbal Learning), one visual task (Visual Learning), and one cross-modal activity (Sound Symbol Learning). Delayed recall measures are 
included for the Story Memory, Verbal Learning, Visual Learning, and Sound Symbol subtests. Additionally, a delayed recognition task is provided for Story Memory. More detailed descriptions of the content of these subtests are presented below, in the Materials section.

It is essential to note that subsequent factor analysis manipulations, some using different statistical procedures than the one employed by the test developers, have indicated alternative factor solutions for the WRAML subtests. For example, Stone (1990), again looking at the original standardization sample ( $\underline{N}=2363$ subjects, aged 5-17 years) and using the same analyses, provided supporting evidence for Sheslow and Adam's (1990) finding that the Visual Learning subtest actually loaded more significantly on a "visual" (than a learning) factor. Further, Story Memory, suggested to be a verbal task, loaded more highly on a "learning" factor. This may reflect the contextual nature of the task. A new finding was that for the older standardization sample age group (9-17 years, versus 5-8 years), the purportedly visual task of Finger Windows actually loaded equally as high on the verbal factor (Stone, 1990). This finding suggests that older subjects, with their more advanced verbal problem-solving abilities, increasingly rely on a language-based mediation system to process and remember the visual sequences.

Gioia (1998) conducted a principal factor analysis with the standardization sample subtest intercorrelation data. This procedure differed from the original analysis in that the common variance between subtests was emphasized; both common variance and the variance specific to an individual subtest were utilized in the original principal components analysis procedure (Sheslow \& Adams, 1990). The more parsimonious strategy of principal factor analysis revealed different groupings of the nine subtests, both in comparison to the components analysis and across the two age groups. The one stable factor across all age groups was a rote verbal repetition factor, involving the Sentence Memory and Number/Letter Memory subtests. 
The other subtests were clustered according to a mixed verbal/visual learning and memory factor, and a visual learning and memory factor, for 5-8 year olds (with Design Memory loading on both factors); and a general learning and memory factor, and a verbal learning and memory factor, for 9-17 year olds (with Verbal Learning loading on both factors). Similar to Stone's (1990) results, Finger Windows loaded on the visual factor for the younger subjects, and on the general (cross-modal) learning and memory factor for the older subject group.

Gioia (1998) noted that, in general, loadings were higher for specific factors of each subtest than for common factors among the subtests, which suggests that scores of individual subtests might be best interpreted alone as opposed to considering overall index scores. In fact, reliability for the subtests was rather strong, with median reliability coefficients ranging from .78 to .90 (Sheslow \& Adams, 1990). Gioia also pointed out that the learning factor, hypothesized to be measured by the three subtests which involve repeated presentations of the same information, did not hold up under this factor analysis. He indicated the difficulties in separating learning (encoding) from memory (information storage and retrieval). The summary score of the four trials, thus, may not accurately reflect how the presented information is "learned" over a short time period. Examination of performance on each learning trial may provide more pertinent information regarding the nature of responding (e.g., demonstration of a learning curve across task trials, primacy versus recency aspects of performance on the word list of the Verbal Learning subtest, and consistency in identified stimuli on the Visual Learning and Sound Symbol subtests). Finally, Gioia (1998) noted the potential problems in computing correlations across broad age ranges (i.e., 5-8 years, 9-17 years). Theories of intellectual and memory development (Schneider \& Pressley, 1989) suggest that more restricted age groupings might enhance the determination of aspects and strategies of remembering that are prevalent at specific 
developmental levels. The finding of the "transition" among young adolescents from "simple" visual processing to additional reliance on a verbal mediation strategy to solve Finger Windows supports this assertion (Gioia, 1998; Stone, 1990).

Other investigations using the normative standardization sample have provided evidence to support some of Gioia's (1998) results. Confirmatory factor analyses of up to nine proposed factor solutions have typically supported a three-factor model that includes a visual memory factor, a verbally-mediated factor, and a short-term attention/memory factor (Burton, Mittenberg, \& Burton, 1993; Donders, 1995; Rogers \& Gridley, 1995; Wasserman \& Cambias, 1993). These findings were replicated in a clinical population of children referred for school performance and behavior problems (Aylward, Gioia, Verhulst, \& Bell, 1994). However, an investigation that evaluated the fit of seven different factorial models for both a clinical and the standardization group actually found that the null model (each subtest serving as a single factor) provided a better fit than any other configuration (Callahan, Haut, Haut, \& Franzen, 1993). This supports Gioia's (1998) assertion that because the WRAML subtests each contribute a large amount of unique variance, it may be more clinically prudent to interpret a subject's performance on each of the subtests separately, as opposed to assumptions based on the global indices or other factors.

Nearly three-quarters of the sample in the Aylward et al. (1994) study, mentioned above, had a diagnosis of ADHD, which the authors noted as significant in terms of a need to analyze the attentional components in the WRAML subtests. In fact, in an earlier report, Haut and her colleagues had found that among children referred to a pediatric neuropsychology service, the Number/Letter and Finger Windows subtests correlated more strongly with other neuropsychological tests of attentional abilities (e.g., the Knox Cube Test (Stone \& Wright, 1981) and the Digit Span subtest of the Wechsler tests (WISC-R, WAIS-R, WISC-III; Wechsler, 
1974, 1981, 1991) than with the WRAML subtests with which they are purportedly aligned (i.e., other subtests included in the Verbal Memory and Visual Memory scales, respectively) (Haut, Haut, \& Franzen, 1992). Further, those two WRAML subtests were not highly correlated with measures of general cognitive functioning, suggesting that those particular tasks address a specific attentional factor. This hypothesis has been confirmed in other investigations (Adams, Robins, et al., 1992; Olds \& Schlieper, 1994; Williams, Griebel, \& Dykman, 1998).

In a second study with a similar clinical sample (Haut, Haut, Callahan, \& Franzen, 1992), an exploratory principal factor analysis using the WRAML subtests, as well as the Knox Cube Test and Digit Span subtest of the WISC-R or WISC-III indicated a two-factor solution: Factor 1, described as a combined/general memory factor, was composed of all the WRAML subtests except Number/Letter Memory, Finger Windows, and Sentence Memory, which contributed to Factor 2, described as an attentional factor, along with the Knox and Digit Span (both forward and backward) tasks. It is interesting to note that the loading of Finger Windows on the attentional factor is different from the Aylward et al. (1994) findings, which indicated that only Number/Letter Memory and Sentence Memory loaded on the attentional/short-term memory factor. This may again be explained by developmental age considerations: the Haut, Haut, Callahan, et al. (1992) study included only adolescents from 9-17 years of age (consistent with ages of the older WRAML standardization group), while Aylward et al. (1994) included only a restricted range of younger children (mean age of 9.86 years). This suggestion is consistent with cautions about interpreting findings without considering developmental issues (Gioia, 1998; Stone, 1990; Wasserman \& Cambias, 1993).

It is also important to note that for the clinical sample in the latter Haut et al. (Haut, Haut, Callahan, et al., 1992) study, which presented with a range of diagnoses such as learning 
difficulties, brain tumors, head injury, seizure disorder, and substance abuse, the general memory factor did not divide along modality lines. This was discrepant from the analyses reported for the standardization sample, which consistently produced a separation into two memory factors by modality (e.g., Donders, 1995). Further investigation of this finding may provide insights regarding profiles of learning, memory, and attention abilities demonstrated by children and adolescents with documented difficulties in those areas, versus individuals without such problems.

The ramifications of this evidence regarding the attentional components of memory, at least as they affect performance on the WRAML, are particularly important to practitioners whose role it is to evaluate the cognitive functioning of children referred for some type of learning or behavioral problem. As Haut and her colleagues noted (Haut, Haut, Callahan, et al., 1992), the contribution of attentional dysfunction must be disentangled, to the extent possible, from memory deficits that potentially reflect a different functional aspect of what "remembering" entails. This concern prompted investigators to begin to evaluate the usefulness of the WRAML in discriminating children and adolescents with ADHD from control children without attentional difficulties.

The WRAML developers and their colleagues indicated that children with "pure" ADHD, that is, no comorbid behavioral or learning problems, were classified with $76 \%$ accuracy according to scores on the WRAML subtests (Adams, Sheslow, Robins, Payne, \& Wilkinson, 1991; Adams, Robins, et al., 1992). Those authors reported that discriminations of the ADHD group from a control group selected from the standardization sample, and matched according to age, gender, and other socioeconomic variables, occurred along the global indices of visual and verbal memory. The Story Memory subtest did not enter into the discriminative analysis, nor did 
the learning subtests. Closer scrutiny of the data revealed that the Finger Windows and

Number/Letter subtests contributed most significantly to the group separations; these findings are consistent with those of Haut and her colleagues (Haut, Haut, \& Franzen, 1992; Haut, Haut, Callahan, et al., 1992). The specific aspects of these two subtests that were hypothesized to be related to attention include the minimal level of meaningfulness of the information and the immediate nature of recall demands, after one presentation of the information (Adams et al., 1991; Adams, Robins, et al., 1992).

Hooper and his colleagues provided some additional evidence of the WRAML's discriminant validity for children with problems of inattention and impulsivity (Hooper, Linz, Tramontana, \& Stein, 1992). Interestingly though, their findings suggested that the Picture Memory and Verbal Learning subtests best discriminated the clinical (subjects with one of the three subtypes of ADHD) and control (typically-functioning) groups. Adams et al. (1991) had, in fact, suggested that tallying the commission errors on the Picture Memory subtest (e.g., the number of items that a child indicated as missing that were inaccurate) might provide a measure of attentional functioning in children, similar to commission errors on other visually-based attentional measures (e.g., the GDS). Haut's group found that in a group of children referred for a variety of neuropsychological concerns, Picture Memory commission errors did correlate significantly with the GDS and the Knox Cube Test, providing some preliminary support for that hypothesis (Kashden, Haut, Wong, \& Franzen, 1994).

Despite these preliminary findings, the size of the clinical samples (ADHD subjects) used in the above studies (Adams et al., 1991; Adams, Robins, et al., 1992; Hooper et al., 1992) was small ( $\mathrm{N}=19,25$, and 32 respectively). Further, the age range of the subjects was not provided for the Adams et al. (1991) study, and only a mean age (8.7 years) was indicated for the Adams, 
Robins, et al., 1992 study. Hooper et al.'s group spanned the broad age range of the standardization sample (5.5 to 17.9 years). Thus the results of these investigations were questionably generalizable, and did not provide compelling evidence regarding the discriminability of the WRAML for school-aged children with ADHD.

Two investigations were identified that attempted to evaluate the utility of the WRAML in discriminating between ADHD and LD groups (mean age $=9.8-9.9$ years in both studies) (Aylward, 1994; Phelps, 1996). It should be noted, though, that Aylward's group was subdivided according to attentional subtypes, with comorbid LDs not controlled for, and that Phelps' LD group only represented reading disabilities. Further, no typically-functioning control group was used in either study. Nonetheless, in their findings, both authors suggested that the WRAML added little to the discrimination of ADHD versus LD children. However, analyses in both of the studies utilized the global index scores rather than the subtest scores. Phelps (1996) did find that the Learning Index provided the least amount of information about acquisition and recall of information, which lends support to the factor analyses that load the "learning" subtests according to the visual or verbal memory requirement involved. She also advocated for the examination of the learning curves demonstrated by subjects across the four trials (on the "learning" subtests), to obtain more clinically useful information about learning and memory. Aylward (1994) called for profiles of test behavior on WRAML subtests (e.g., reaction times, processing speed) in order to determine the relevance of WRAML information to clinical diagnosis of ADHD or LD. In both studies, however, very different findings may have been obtained if individual subtest scores had been used in the data analysis, as proposed by Gioia (1998) and other investigators. Clearly, these studies did not provide strong evidence about the 
utility of the WRAML in discriminating ADHD versus LD populations, and additional investigations appear to be warranted.

The WRAML's ability to identify specific memory deficits in pediatric medical populations has been demonstrated in several studies; for example, unique performance profiles have been identified for children with epilepsy (Haut, Williams, \& Hendon, 1994; Warner, 1992; Williams, Sharp, \& Griebel, 1992), and children with leukemia who have undergone intrathecal chemotherapy (Hill, Ciesielski, Sethre-Hofstad, Duncan, \& Lorenzi, 1997). Children and adolescents with a history of traumatic head injury also appear to demonstrate particular deficits in memory functioning as measured by the WRAML (Driscoll, 1992; Farmer et al., 1999; Haut, Williams et al., 1994; Ong, Chandran, Zasmani, \& Lye, 1998; Williams \& Haut, 1995). These studies have documented deficits in both verbal and visuo-spatial learning and recall abilities for children post-TBI relative to typically-developing control groups. Specific deficits in delayed recall of non-contextual verbal information (e.g., Verbal Learning subtest) appears to be a common characteristic across levels of injury severity and age ranges, while immediate recall and recognition are commonly intact for children with mild to moderate injuries. Severe brain injuries are typically associated with a variety of aspects of verbal memory deficits. Visual and spatial memory tasks have also been found to be related to severity of injury, with more severely impaired children showing deficits on several visual-spatial tasks and mildly to moderately impaired children showing deficits only on visual reproduction tasks relative to noninjured, typically-functioning peers. Overall, there is some evidence to suggest more significant visualspatial memory deficits for severely brain-injured children than verbal recall deficits (Farmer et al., 1999). 
In sum, the WRAML appears to be a comprehensive, well-standardized (see data in Materials section, below) measure of memory in children, particularly in comparison to adultnormed assessment tools from which memory abilities had previously been estimated. With continued application in clinical settings, the appropriate uses, and limitations, of this instrument can be further identified. That call for a circumscription of the WRAML's utility was one of the purposes of the present investigation.

Purpose of the Present Study

Reasons to further investigate the utility of the GDS and the WRAML. The Gordon Diagnostic System (GDS; Gordon, 1983) is a computerized tool designed to measure cognitive and behavioral impulsivity, as well as sustained attention and effects of distractors on selective attention. A substantial body of published research supports the clinical efficacy of this instrument in discriminating children and adolescents with problems in the skill areas assessed. Fewer investigations have documented the GDS' discriminative validity in separating ADHD from LD children (Aylward et al., 1990; Robins, 1992). No studies were identified that used the GDS to evaluate attentional abilities in children who have sustained a TBI. Although some investigations have indicated intercorrelations between the GDS and various intellectual and achievement tests (Gordon et al., 1990; Grant et al., 1990), no studies were identified in the current literature review regarding the relation between the GDS and measures of children's memory, such as the WRAML.

The Wide Range Assessment of Memory and Learning (WRAML; Sheslow \& Adams, 1990) is an evaluation tool standardized for use with children and adolescents aged 5-17, inclusive. Although conceptualizations of the specific deficits in ADHD have conspicuously lacked discussions of the importance of memory in relation to attentional processes, a number of 
investigators have suggested that some WRAML subtests are more strongly correlated with measures of attention than of memory per se (Burton et al., 1993; Haut, Haut, Callahan, et al., 1992). This becomes relevant as the uninformed user of the WRAML may, for example, interpret deficient performance on the WRAML Verbal Memory Index as reflective of globally impaired verbal memory, when the child's pattern of performance on the verbal memory subtests actually includes intact abilities on Story Memory, but impaired performance on Number/Letter and Sentence Memory subtests. Given that research has demonstrated apparent attentional components involved in the Sentence Memory and Number/Letter subtests (e.g., Haut, Haut, \& Franzen, 1992), the more appropriate conclusion may be that the child is demonstrating deficits with aspects of auditory-verbal attentional functioning, but evidencing intact verbal memory for semantically-related discourse. A similar concern exists for the Visual Memory Index, in which particularly deficient performance on the Finger Windows subtest may lead to the erroneous clinical conclusion that visual memory is impaired, though the impairment actually may lie in deficient attention to visual-spatial sequences.

These types of clinical judgments are likely to be made if the clinician relies on information presented in the WRAML manual only (Sheslow \& Adams, 1990), without considering research completed subsequent to its publication. Although that research, as reviewed earlier in this paper, provides evidence for a potential attentional "link" or "factor" involved in the WRAML, much of the data have been based on confirmatory or exploratory factor analytic studies (Gioia, 1998; Haut, Haut, Callahan, et al., 1992; Stone, 1990). Few studies exist which actually compare performances of children with and without attentional difficulties on the WRAML (e.g., Adams et al., 1991; Adams, Robins, et al., 1992; Hooper et al., 1992). Similarly, only two studies were identified that examined the discriminability of the 
WRAML in identifying children with ADHD versus LD (Aylward, 1994; Phelps, 1996). In a few studies, children with TBI have also been shown to demonstrate specific profiles of deficits on the WRAML (e.g., Farmer et al., 1999; Williams \& Haut, 1995); this clinical group provides a good comparison group for ADHD and LD children due to their concomitant difficulties with attention and memory. It was a goal of this investigation to add to the literature regarding the clinical utility of the WRAML with these particular clinical populations of children. Further, it was thought that an investigation of the relations between performance on the WRAML and the GDS might assist in clarifying the theoretical association between attentional and memory functioning.

Research questions. The first question addressed in this research investigation was the correlation between measures of impulsivity, vigilance, and distractibility on the GDS, and visual and verbal memory and attentional factors on the WRAML. Specifically, Hypothesis I asserted that performance on the GDS tasks, which purportedly measure specific aspects of attentional functioning, would be positively correlated with the WRAML subtests which have a suggested attentional component (i.e., the Finger Windows, Number/Letter Memory, and Sentence Memory subtests). Further, performance on the Knox Cube Test and Digit Span subtest (of the WISC-III) was expected to be significantly correlated with those WRAML subtests as well as the GDS tasks. Both the Knox Cube Test and the WISC-III Digit Span subtest have been found to measure attentional functioning (Anastopoulos, Spisto, \& Maher, 1994; Bornstein, 1983; Karatekin \& Asarnow, 1998). This hypothesis was addressed statistically through a canonical correlation analysis, as well as follow-up correlations between significant canonical variables. 
The second research question was related to the high rate of comorbidity of learning disorders in children with attentional problems (McGee \& Share, 1988, Semrud-Clikeman et al., 1992). It is important to distinguish the specific nature of a given child's problems with attention and related difficulties, particularly in terms of implications for treatment. A child who demonstrates inconsistent attention during relatively abstract language-based activities but is not distractible, and in fact excels, with tasks that are manipulative and concrete in nature may actually be demonstrating a specific language disorder. The intervention strategies for this type of diagnosis would clearly be different than if the determination was that the child was having trouble attending to tasks across settings, but generally demonstrated adequate intellectual and academic functioning. It should also be noted that the prevalence of children with "pure" ADHD, without LD, is probably much lower than the current epidemiological figures suggest (e.g., Barkley et al., 1990; Cantwell, 1996; Safer \& Allen, 1976). Thus, diagnostically and prognostically, it would be helpful to be able to identify any differences in attentional and memory profiles of children with ADHD only, versus those who also demonstrate specific learning disorders. Children with TBI represented a third clinical group that could be used for comparison purposes, in order to determine any differences between the structurally-based attention deficits demonstrated by the TBI group, and the more functionally-based difficulties demonstrated by children with ADHD. Typically-developing children matched for age and IQ were used as a Control group.

Thus, the second part of this study attempted to discriminate and classify those subjects according to their appropriate clinical (ADHD, LD/ADHD, TBI), or Control (typically developing) group, based on their performances on the combined battery of tests. Comparisons of group performances on the WRAML and the GDS were also conducted, to determine any 
differences in performance profiles between groups. Based on research reviewed above, Hypothesis II predicted that children with "pure" ADHD would demonstrate more difficulties on the GDS and WRAML attention-related subtests than individuals with LD and ADHD, but that the latter group would perform less well than the "pure" ADHD group on other measures of memory and learning. Subjects in the TBI group were expected to demonstrate more difficulties with the GDS and WRAML visual-spatial tasks than with verbal learning and memory tasks, based on findings reported in previous investigations for children and adolescents with severe brain injuries (e.g., Farmer et al., 1999). All three clinical groups were expected to perform less well on all of the measures, compared to the Control group. Direct discriminant function analysis was used to address this hypothesis. 


\section{$\underline{\text { Subjects }}$}

The 75 clinical subjects were drawn from consecutive referrals to the neuropsychological service of the Department of Behavioral Medicine and Psychiatry, West Virginia University School of Medicine. Given that the first conjoint administration of the WRAML and the Gordon Diagnostic System with referred patients occurred in July 1990 in this clinic, archival data from July 1 of that year until December 1998 were reviewed for potential inclusion in this investigation. Specifically, clinical subjects were considered for the study if they met the following criteria: (a) chronological age was between 9 years, 0 months and 14 years, 11 months; (b) some measure of intellectual functioning was available in the referral records or from the evaluation at WVU; and (c) all targeted measures for the study were administered. The criterion for age inclusion reflects (a) the pooling of a homogenous subject group for scoring purposes, given specific age-related changes in norm groups across the different tests utilized in the study; and (b) an attempt to eliminate some of the confounding factors regarding developmental considerations (Gioia, 1998; Wasserman \& Cambias, 1993) and environmental issues (e.g., most children in this age range were enrolled in upper elementary and middle school grades). The second inclusion criterion reflects an effort to control for differences in intellectual functioning as a source of variance in the results; all clinical subjects had confirmed or estimated intellectual performance in the average range of functioning.

Confirmed or provisional diagnoses were used to divide the clinical sample into each of three groups of 25 subjects. Group 1 consisted of children with a DSM-IV (APA, 1994) diagnosis of Attention Deficit/Hyperactivity Disorder (ADHD) only (any of the three subtypes), based on referral and historical reports of behavioral functioning. Children described as 
demonstrating a Learning Disability in addition to ADHD (LD/ADHD) comprised Group 2. The same procedures as indicated for Group 1 were used to identify an ADHD diagnosis. Learning disabilities were documented through psychoeducational reports in the referral records, or through the testing conducted by the Neuropsychology Service at WVU. Two strategies were used to identify learning disabilities: (a) DSM-IV criteria for specific learning disorders (i.e., achievement scores in a specific skill area at least two standard deviations below assessed intellectual potential), and/or (b) documentation of a significant discrepancy between Verbal and Performance IQ scores (i.e., specific verbal or nonverbal learning disability). Children with the latter types of cognitive profiles are considered to be learning disabled according to current psychological and educational literature (Denckla, 1996; Harnadek \& Rourke, 1994). Further, that subgroup of children clearly represents a different type of learning profile than the "pure" ADHD group (August \& Garfinkel, 1989, 1990; Kataria et al., 1992; Korkman \& Pesonen, 1994). Thus both methods of identifying children with LDs were thought to be appropriate for the present study. Group 3 consisted of children who had sustained a traumatic brain injury (TBI), as documented in medical and historical records. For this group, a pre-morbid diagnosis of ADHD ruled out inclusion in the study.

An independent rater reviewed the records of each proposed clinical subject (identified by the author, from the files of the Pediatric Neuropsychology Service), and determined group placement based on the criteria described above. [Although subtype of ADHD was calculated by the independent rater based on review of the behavioral records, this information was not entered into the data analysis, due to the small sample sizes.] A total of 69 records of children with a diagnosis of ADHD were reviewed prior to identifying 25 subjects who had "pure ADHD" and 
25 subjects who had an ADHD diagnosis with a comorbid Learning Disability. A total of 30 records of children with a TBI were reviewed prior to identifying the 25 clinical subjects used.

For each of the 75 subjects selected for the clinical sample, the following information was documented from the hospital records: (a) confirmed or estimated level of intellectual and academic functioning, and the method used to determine that information (i.e., from school records or clinical psychoeducational reports); (b) diagnoses or learning/behavioral descriptors (e.g., type of learning disability) indicated at the time of referral and at the completion of the neuropsychological evaluation (NOTE: the latter information was only used to disqualify subjects from the study if the final diagnosis ruled out ADHD or LD for Groups 1 and 2); (c) methods employed to obtain those diagnoses or descriptors; (d) significant medical/educational history; (e) any prescribed medications, and presence or absence of medication at the time of test administration; (f) school placement (grade, setting, retention, etc.); (g) gender of the subject; (h) age of the subject; and (i) race of the subject.

Additionally, the following information was documented for the TBI group: (a) preinjury educational, medical, and behavioral history; (b) type of accident; (c) medical information at the time of and immediately following injury (e.g., Glasgow Coma Scale score, length of loss of consciousness (LOC), findings on CT or other brain scan); (d) post-injury seizure activity; and (e) post-injury educational and behavioral status. It should again be noted that individuals in the TBI group who had a pre-injury diagnosis of ADHD were not included in the present study, although pre-morbid learning disabilities did not exclude a subject. Four of the TBI subjects had documented, pre-injury verbal learning disabilities, and two were reported to have performance/nonverbal learning difficulties. Pre-morbid intellectual functioning was in the average range for all TBI subjects. 
A control group of 25 subjects matched for age in years was also included in the study. Based on information gathered from a history questionnaire completed by parents (see Appendix A), these children demonstrated neither present nor historical evidence of attentional, behavioral, or learning problems. Further, no previous TBI was reported for any of the control subjects. School achievement was determined through review of recent report cards, and parent and child verbal report. A brief version of a standardized test of intellectual functioning was administered to these participants to determine estimated intellectual functioning. A four-subtest short form of the WISC-III (Wechsler, 1991), recommended by Sattler (1992) as a screening device, included Information and Vocabulary as Verbal subtests, and Picture Completion and Block Design as Performance subtests. Reliability coefficient for this short form was reported as $\underline{r}=.936$, with a validity coefficient of .889. These coefficients were derived by Sattler (1992) using the internal consistency reliability data and subtest intercorrelations, from the standardization sample, for the subtests included on the short-form. Thus, the coefficients do not simply reflect concurrent validity with the Full Scale IQ score, but rather use information about the specific subtests that make up the short-form, to determine the reliability and validity of that particular grouping of subtests. Given that the obtained information was only used to screen the control subjects for general intellectual functioning, the selected short-form measure appeared to be clinically useful. Scaled scores for the four subtests were totaled, and converted to Deviation Quotients (as reported by Sattler, 1992). Only those children scoring in the average range on this testing (defined as estimated Deviation Quotient falling between 85 and 115) were included in the study, to provide an intellectually normative control sample. A total of 44 individuals were assessed in order to identify 25 children and adolescents who met this inclusion criteria for the control group (i.e., many volunteers scored in the above average range of intellectual functioning). 
Control group subjects were recruited through a flyer posted around the WVU hospitals and medical school, as well as the Department of Psychology. Interested individuals contacted the principal investigator directly, and a brief screening was conducted to determine whether the basic inclusion criteria were met. Potential participants were then scheduled for an assessment session. Parents of control group participants were asked to complete a data questionnaire (see Appendix A), as well as a university-approved (from the West Virginia University Institutional Review Board) consent form. Assent for participation was also obtained from each control group subject. No control subjects who volunteered to participate in the study later elected to withdraw their participation or discontinue the testing session. Control subjects were paid \$20.00 to compensate them for their investment of time; all control subjects completed the testing within one $2-2 \frac{1}{2}$ hour session.

Table 1 presents a review of the important subject characteristics for the three clinical groups, and for the typically-functioning control group. Subjects were matched for race, age, and intellectual functioning (based on pre-morbid intellectual testing for the TBI group). Gender groupings were different for the clinical groups versus the control group, with a preponderance of boys in each of the three clinical groups and a reverse majority in the control group (i.e., twothirds of the group were girls). However this variable was not considered to be a confound for this study, because none of the measures utilized had identified significant effects for gender (as reviewed in the WISC-III, WRAML, and GDS administration manuals; Wechsler, 1991; Sheslow \& Adams, 1990; Gordon \& Mettelman, 1988, respectively). Educational support, in terms of special or compensatory education classes (for academic and/or behavioral support, or both), was documented for a majority ( $88 \%$ ) of the LD/ADHD subjects, versus only half of the ADHD group, and less than one-third of the TBI group. It should be noted that for 
approximately one-third of the TBI group, documented intelligence scores fell to the low average to borderline range post-injury (subjects whose intellectual functioning fell below the borderline range post-injury were excluded from the study), and four of the subjects who had documented pre-injury LDs reported further declines in academic functioning post-injury. Despite these findings though, previous grade retention was relatively equivalent across the three clinical groups.

Numbers of subjects in ADHD subgroups were similar across the two groups with this diagnosis; however, due to the small sample sizes, these variables were not entered into the analysis. Twice as many LD/ADHD subjects were suspected of having additional comorbid diagnoses, compared to "pure" ADHD subjects; symptoms included characteristics of oppositional defiant disorder, psychosocial stressors, and depression. However, none of the diagnoses was confirmed. Nine of the subjects in the TBI group had similar suspected diagnoses post-injury. Finally, the finding that five of the ADHD subjects were taking psychostimulant medication on the day of the evaluation for this study is important in terms of interpreting results; the medication may have masked some of the difficulties otherwise displayed by the subjects. Only two subjects in each of the other two clinical groups were taking the same types of medications on the day of the evaluation.

Table 2 reviews the specific injury characteristics of the TBI group. Half of the subjects had been involved in a motor vehicle accident, either as a passenger in a vehicle or as a pedestrian or bicycling victim (hit by a motor vehicle). Nearly a third of the subjects had been involved in an isolated bicycle or all-terrain vehicle accident (not related to a motor vehicle). The remaining subjects had suffered a fall or an assault. Injury severity was determined by reviewing several pieces of information. Glasgow Coma Scale scores (GCS; Teasdale \& Jennett, 
1974), typically considered to be one of the best indicators of injury severity (Fletcher et al., 1995), were available for only 8 of the 25 TBI subjects. Four of those subjects had scores in the mild range, three in the moderate range, and one in the severe range. Another potential index of severity is length of LOC (Fletcher et al., 1995). Of the eight subjects for whom length of LOC was reported, three had time spans of over 24 hours; five of the others ranged from less than 15 minutes to 3 hours. Eight other subjects reported no LOC, and for nine subjects, presence or length of LOC was unknown. Unfortunately none of the patients with significant LOC had GCS scores reported in the medical records, thus it was impossible to compare those findings for any subjects. Documentation of structural damage revealed that 19 of the subjects had positive CT or MRI findings, with the remaining six reporting findings within normal limits or no findings (e.g., tests not conducted or else not reported). William, Levin, and Eisenberg (1990) suggested that positive brain scans were indicative of at least moderate injuries; thus, this information appears to be the best way to classify the TBI group in this study. In sum then, this TBI sample should be considered as having sustained predominantly moderate brain injuries.

Table 3 reviews the specific characteristics of the learning disabilities in the LD/ADHD group. In the present sample, a little over a third of the subjects demonstrated a nonverbal learning disability. Six had verbal learning disabilities, as documented in a significant verbal/performance discrepancy on the WISC-III or WISC-R, and six others had been diagnosed with reading and spelling LDs. Two subjects each were described as having predominantly math or written language learning deficits. An expected finding was that most of the subjects with a nonverbal learning disability had weak achievement scores primarily in the areas of math and written language, while the subjects with a verbal learning disability displayed more problems with reading and spelling. 
$\underline{\text { Materials }}$

Wide Range Assessment of Memory and Learning (WRAML). The WRAML provides a measure of information acquisition and recall in children and adolescents. The standardization age range was 5 through 17 years. Three subtests are presented within each of three composite scales: Verbal Memory, Visual Memory, and Learning. A brief description of each subtest follows, based on information from the WRAML Administration Manual and materials (Sheslow \& Adams, 1990).

On both the Verbal and Visual Memory scales, subtests represent a continuum of meaningfulness for the information presented. Verbal scale subtests will be reviewed first, followed by the Visual scale subtests. At the most basic and rote level of the verbal memory tasks, the Number/Letter Memory subtest requires the child to repeat series of randomly intermixed numbers (1-9) and letters (A-Z). The task progresses from two units (e.g., "2-S") through ten units (e.g., "8-4-R-2-Y-Q-9-A-2-S"), with varying numbers of trials for each unit length (ranging from one trial for two and ten units, to six trials for six units). Next on the verbal information continuum is Sentence Memory, in which the individual is asked to repeat, verbatim, progressively longer sentences that are designed to be contextually familiar and relevant to children. Items increase from a length of three words ("I like pizza.") to twenty-five words. The most meaningful verbal information is provided in the Story Memory subtest. Following verbal presentation of each story by the examiner, the individual is immediately asked to repeat as many parts of the story as possible. Three stories, each of increasing length and complexity, are provided: the first (i.e., birthday party, 22 units of pertinent information) and second (i.e., fishing, 23 units of pertinent information) are read to children aged 5-8 years, the second and third (i.e., getting a job, 28 units of pertinent information) are presented to the older age group 
(9-17 year olds). Although the WRAML manual (Sheslow \& Adams, 1990) indicates that the stories were purposefully developed along different levels of interest and linguistic complexity, there is some evidence that the third story (i.e., getting a job) involves such a significant increase in linguistic and semantic complexity that the overall subtest score for Story Memory, for older subjects, is not very meaningful (Haut, Warren, \& Bretzman, 1994). Rather, those authors suggested that it is more appropriate to consider the child's performance on each of the stories separately, when interpreting test findings.

Of the visual memory tasks, Finger Windows involves rote and minimally meaningful task demands. The child is required to repeat progressively longer series of spatial sequences demonstrated by the examiner: a pencil (eraser end) is placed through a consecutive series of circular openings from the back of a sturdy plastic $81 / 2 \times 11$ inch card, and the child must repeat each series by placing his/her finger through corresponding openings from the front side of the card. The sequences range from two to nine units, with varying numbers of trials for each unit length (ranging from one trial for eight and nine units, to six trials for four and five units). Children aged 5-8 years start at the two-unit level, while older children start at the three-unit level. Next on the visual information continuum is the Design Memory subtest, which involves reproducing familiar geometric figures with paper and pencil (e.g., circles, triangles, rectangles). The task requirement is to draw specific arrangements of the figures, after looking at a sample stimulus card for five seconds. A 10-second delay occurs before the child can begin drawing the figure arrangements. A total of four different arrangements are presented to all age groups. A "pre-test" (i.e., copying each of the figures separately) is provided to enable the examiner to determine the basic integrity of the child's visual-motor skills; thus, scoring for the subtest reflects "memory" only, in that children are not penalized for poor visual-motor (i.e., drawing) 
skills. The most complex and meaningful of the visual memory subtests is Picture Memory, which requires the child to study a content-relevant picture for 10 seconds, and then identify in an immediately-presented second picture any items that have been changed or added. The number of altered items ranges from 5 to 17 across the four stimulus pictures.

All subtests on the Learning scale involve repeated presentations of information, over four trial sets. The Verbal Learning subtest was adapted from Rey (1958, in Sheslow \& Adams, 1990); the child is asked to immediately repeat, in a free-recall paradigm, a list of words read to him. Thirteen words are presented to children aged 5-8 years; three additional words, for a total of 16, are presented to older children. The word list is presented four times, each time in the same order; each presentation is followed by a recall period. On the Visual Learning subtest, the child is required to recall the spatial location of colored, geometric designs on a stimulus board. Fourteen different designs are each presented in a 2-square inch inset "box", in a 4 by 4 matrix (i.e., four horizontal rows of four designs). However, two of the squares are "blacked out" for the older age group (the third square in the second row, and the second square in the third row, moving from left to right), to leave 14 stimulus items; all four of the middle squares are blacked out for the 5-8 year old age group, to leave only 12 stimulus items. Each design to be used is hidden from view by a foam cover. An initial training trial is implemented to reveal each of the stimuli on the board, one at a time, for one second. A flip-card booklet is then used to present one of the designs at a time, and the child is asked to point to the location on the board where the matching design is located (without raising the cover for the item). Order of presentation of each design is randomized across the four trial sets. The third Learning subtest, Sound Symbol, involves a paired-associate learning task. Following an initial training trial, children are asked to verbally recall the nonsense sound associated with a particular abstract figure, presented in 
random order on individual picture cards in a flip-card booklet. Younger children are exposed to eight figures and nonsense sounds; older children are exposed to 12. To encourage learning on the Visual Learning and Sound Symbol subtests, three sets of trials are presented with immediate feedback for each item (as to the correctness of the response) and correction procedures (revealing the correct design location on the Visual Learning task; providing the correct sound on the Sound Symbol task). The fourth set of trials on each of those subtests involves no feedback nor corrections, in order to limit "contamination" on a later delayed recall trial.

Delayed recall subtests are included for Verbal Learning (typically after a 10-minute delay) and Visual Learning, Sound Symbol, and Story Memory (typically after a 30-40 minute delay for each subtest). Task demands remain the same as for the original administration of each subtest, to assess for recall of information over time. For Story Memory, an additional recognition task is available, which presents 15 questions each for two of the stories, in a threeitem multiple-choice format. Younger children are asked questions about the second story (i.e., fishing); older children are asked questions about the third story (i.e., getting a job).

The subtests are varied in order of presentation across the three scales, though the administration order has been standardized to remain constant. The specific order of subtest administration is: Picture Memory, Design Memory, Verbal Learning, Story Memory, Delayed Recall -- Verbal Learning, Finger Windows, Sound Symbol, Sentence Memory, Visual Learning, Number/Letter Memory, Delayed Recall -- Sound Symbol, Delayed Recall -- Visual Learning, Delayed Recall -- Story Memory, and Story Recognition. Several subtests have a discontinue criterion, following three consecutive errors. Total time for WRAML administration is typically 1 to $11 / 2$ hours. 
Technical aspects of the WRAML. Raw scores for the nine memory and learning

subtests are converted to scaled scores, with a mean of 10 and a standard deviation of 2. Age of the child is accounted for in this transformation, across 21 different age groupings (half-year intervals for children through age 13 years, full year interval for 14 year olds, and two year interval for 16 and 17 year olds). For the WRAML standardization sample, median standard error of measurement ranged from .9 to 1.3 for the subtest scores. The three subtests that make up each of the three scale indices (Verbal Memory, Visual Memory, and Learning) are combined to yield the overall Index scores; the three Index scores added together (and thus all nine subtest scale scores) produce a General Memory Index (GMI) score. Mean score for the indices is 100, with a standard deviation of 15 . Median standard error of measurement for the three scale indices ranged from 3.9 to 4.7; for the General Memory Index the median standard error of measurement was 3.0. Confidence bands were reported as $68 \%$ for one standard error of measurement, and 95\% for two standard errors of measurement (Sheslow \& Adams, 1990).

Scores for the Delay tasks are presented only as "difference" scores on the test protocol (i.e., difference between the scores on the Delay task and the fourth trial of the learning/memory task). Scores for the Story Recognition task are presented as raw numbers on the protocol. Both sets of scores are then divided into descriptive ranges based on conventional statistical and clinical judgments (Sheslow \& Adams, 1990). The descriptive categories include atypical, borderline, low average, average, and bright average. Different cut-off scores for the descriptive categories were identified for each of the two primary age groups for the WRAML (8 and younger; 9 and older). For purposes of the present study however, the raw scores from the Delay and Story Recognition tasks were converted to $\underline{\mathrm{z}}$-scores, based on mean and standard deviation scores for those subtests, indicated in supplemental norms provided by the test developers 
(Adams, Sheslow, \& Wilkinson, 1992). It should be noted that only Recognition scores from the third story were used in the data analysis for the present study, based on administrative guidelines for the age group utilized in this project.

Over 2300 children and adolescents were included in the WRAML standardization sample; demographic characteristics such as age (110 or more individuals in each of the 21 age groupings), gender, ethnicity, regional and populational residency (across the United States, in metropolitan and rural areas), and socioeconomic factors were reported to be adequately controlled (Sheslow \& Adams, 1990). A number of reliability and validity measures were completed with this sample.

Median reliability coefficient for internal consistency on the General Memory Index was .96 ; median coefficients were $.93, .90$, and .91 for the Verbal Memory, Visual Memory, and Learning Indices, respectively. Median reliability coefficients for the subtests ranged from .78 (Verbal Learning) to .90 (Sound Symbol). Test-retest reliability (intervals ranging from 61 to 267 days) was .84 for the General Memory Index, and .82, .61, and .81 for the Verbal Memory, Visual Memory, and Learning Indices, respectively. Interscorer reliability was .996 between two independent raters over 82 cases (Sheslow \& Adams, 1990).

In terms of content validity, two original item analysis investigations included 14 subtests, administered to a total of over 400 individuals across three age levels (ranging from 1st grade through high school) (Sheslow \& Adams, 1990). Preliminary findings provided a basis for editing many items and actually discarding five subtests. Item and person separation statistics (ranges $=.99$ to 1.0 , and .79 to .94 , respectively) for the remaining nine subtests appear to adequately differentiate items that define the variables measured, as well as individuals' 
performances according to skill levels (based on Rasch measurement, Wright \& Stone, 1989, in Sheslow \& Adams, 1990). Item bias was reported to be negligible for gender and racial issues.

Evidence was also provided in the WRAML Administration Manual for the criterionreferenced validity of this test, in terms of the overall domain (i.e., memory) under study (Sheslow \& Adams, 1990). Fairly high correlations (i.e., $\underline{r}=.90$ and .72 , respectively) were found between the WRAML Verbal Memory and General Memory Indices and the McCarthy Memory Index (part of the McCarthy Scales of Children's Abilities; McCarthy, 1972) for children aged 67 years $(\underline{N}=41)$, and the WRAML General Memory Index and the Stanford-Binet - 4th Edition, Short-Term Memory Scale (part of the Stanford-Binet Intelligence Scale, 4th Ed.; Thorndike, Hagen, \& Sattler, 1986) (i.e., $\underline{\mathrm{r}}=.80)$ for children aged 10-11 years $(\underline{\mathrm{N}}=50)$. Moderate correlations (i.e., range of correlations: $\underline{r}=.32$ to .63) were obtained between all index scores on the WRAML and the domain scores on the Wechsler Memory Scale-Revised (Wechsler, 1987) for adolescents aged 16-17 years $(\underline{N}=71)$.

Construct validity was measured according to five criteria. First, correlations between subtest scores and age of individuals were significant and positive, and mean subtest scores across age ranges revealed a steady increase in performance. These data were cited in support of a developmental hypothesis regarding measurement of memory (Sheslow \& Adams, 1990).

Second, intercorrelations among the WRAML subtests were significant and positive, suggesting that the various abilities measured by the subtests are related to each other on a memory dimension. It should be noted though that all of those correlations were in the low to moderate range (range: $\underline{r}=.105$ to .605 ), indicating that various subtests measure somewhat different aspects of memory functioning. 
Third, factor structure for the nine subtests was assessed through a principal components analysis, revealing the three scale model (Sheslow \& Adams, 1990). The subtest loadings were not a "perfect fit" with that model however, in that Visual Learning had stronger correlations with the visual memory component, and Story Memory loaded more on the learning component (see also previous discussion of this issue: e.g., Gioia, 1998; Stone, 1990).

Fourth, the relation between the WRAML's measures of memory and general intellectual ability were analyzed. The WRAML authors reported that for younger children (ages 6-0 through 8-11, $\underline{N}=40)$, the WRAML Verbal Memory Index correlated moderately with Verbal IQ $(\underline{\mathrm{r}}=.44)$ and Full Scale IQ $(\underline{\mathrm{r}}=.38)$ on the WISC-R (Wechsler, 1974), and that the Visual Memory Index correlated moderately with WISC-R Performance IQ ( $\underline{\mathrm{r}}=.508)$ and Full Scale IQ ( $\underline{\mathrm{r}}=.456)$ (Sheslow \& Adams, 1990). The correlation between the General Memory Index on the WRAML and Full Scale IQ on the WISC-R was .558. All these correlations were significant at the $\mathrm{p}<.05$ level. As the authors noted, the moderate level of correlations was expected, due to the differences between the constructs of general ability and memory (Sheslow \& Adams, 1990). Unfortunately, these findings were not based on a large sample size, nor was the memorycognitive correlation analyzed for the older age group.

However, a few investigators have attempted to further address those issues. For example, in a study with a larger sample size $(\underline{\mathrm{N}}=141)$ but a broader age range (6-16 years) and a varied referral population (referrals to a neuropsychological service in a pediatric hospital), Olds and Schlieper (1994) supported at least moderate relations between verbal and visual/performance factors across the WRAML and WISC-III (Wechsler, 1991) subtests, with the exception of Finger Windows and Number/Letter (from the WRAML) and Digit Span (from the WISC-III) (these latter three subtests formed a third, auditory-verbal short-term memory 
factor). Interestingly though, the verbal factor accounted for significantly more of the variance than did the nonverbal/visual factor (37.8 versus $9.5 \%$ for the principal components analysis). Similarly, in a clinical sample of children $(\underline{N}=28$, age range $=7-17)$ with intractable seizure disorders, Warner (1992) found support for the relation between WRAML Verbal Memory measures and Verbal IQ ( $\underline{r}=.87)$ and Full Scale IQ ( $\underline{r}=.83)$ on the Wechsler tests (WAIS-R and WISC-R; Wechsler, 1981, 1974, respectively). Further, all of the Wechsler IQ scores (Verbal, Performance, and Full Scale) correlated positively with the WRAML General Memory Index (range: $\underline{\mathrm{r}}$ range $=.73$ to .78 ). However, Verbal Memory also correlated at a higher level with Performance IQ on the Wechsler tests than did Visual Memory ( $\underline{r}=.68$ versus .54 , respectively). Further, there were no significant correlations between performance on any of the WRAML delay tasks and the Wechsler subtest or scale scores.

Haut and her colleagues did not replicate those patterns of findings with a clinical sample $(\underline{N}=100)$ of children and adolescents (age range $=7-17$ years) referred for neuropsychological evaluation based on a variety of diagnoses (e.g., traumatic brain injury, CNS tumors, substance abuse, and psychiatric disorders) (McKittrick, Haut, Schauss, Bradlyn, \& Franzen, 1994). Combining results across three Wechsler tests (WAIS-R, WISC-R, WISC-III; Wechsler, 1981, 1974, 1991, respectively) in a regression equation, results provided evidence that overall intellectual functioning accounted for $42 \%$ of the variance in WRAML General Memory Index scores. However, the correlations between Verbal IQ and Verbal Memory (and Performance IQ and Visual Memory) were not supported. In fact, the IQ scores predicted only minimal amounts of the variance in the WRAML index scores $(.55 \%$ of the variance for Verbal Learning, and $.66 \%$ of the variance for Visual Learning). These findings suggest that intellectual functioning appears to predict general memory ability, but not specific aspects of memory functioning, at 
least in a clinical sample of children who demonstrated problems with attention, learning, and behavior.

Taken together, documented evidence raises questions about the correlation between memory and cognitive ability, in terms of the construct validity of the WRAML as a measure of memory in children.

Finally, the WRAML Manual provides indicators of the relation between memory, as measured by the WRAML, and academic achievement. Sheslow and Adams (1990) reported that for children aged 6-0 through 8-11 $(\underline{N}=40)$, the WRAML General Memory, Visual Memory, and Learning indices were significantly correlated with the three areas (reading, arithmetic, and spelling) assessed on the Wide Range Achievement Test-R (WRAT-R; Jastak \& Wilkinson, 1984). For older adolescents, aged 16 through $17(\underline{N}=33)$, Verbal Memory was significantly related to the three WRAT-R subtests, but the General Memory and Learning indices were only related to arithmetic. These data provide some support for the hypothesis that developmental issues are important in considering the relation of memory to achievement.

Warner's (1992) clinical sample of children and adolescents with seizure disorders demonstrated moderate (though significant) correlations between most of the WRAML and WRAT-R index scores. However, this data is difficult to compare to the above findings because the age range in this latter sample spanned the years 7-17. Olds and Schlieper (1994) indicated that subtest scores of the WRAML predicted academic achievement on the WRAT-R area scores to a greater degree than did the WRAML index scores. Further, different groups of WRAML subtests were more strongly correlated with academic achievement at different ages of the children, which again speaks to the importance of developmental considerations in measuring memory. 
These findings also question the relation between academic learning and memory ability, in terms of construct validity for the WRAML. An interesting outcome of these investigations, though, was the further documentation of the utility of WRAML subtest scores versus index scores in interpreting performance (Callahan et al., 1993; Gioia, 1998).

A shortened form of the WRAML was proposed by the test developers (Sheslow \& Adams, 1990), to be used as a screening measure. The four subtests selected on the basis of the uniqueness of the factors measured, combined with their overall correlation with the complete WRAML form included Picture Memory, Design Memory, Verbal Learning, and Story Memory. Correlations between these screening subtests and the full WRAML protocol were reportedly $\underline{\mathrm{r}}=.846(\underline{\mathrm{p}}<.001)$ for children ages 8 years and younger, and $\underline{\mathrm{r}}=.864(\mathrm{p}<.001)$ for the older group from the standardization sample (Sheslow \& Adams, 1990). Two subsequent investigations, however, have reported skewed results based on the screening measure, with significantly higher estimates of memory functioning for referred children (Kaufmann \& Espy, 1992; Kennedy \& Guilmette, 1995). Further, those authors pointed out that there are no specific guidelines provided in the WRAML Manual for how to interpret the screening findings, in order to make an appropriate decision about the need for further assessment. Thus, the screening instrument appears to have poor clinical utility for referred children. This seems to be particularly true for individuals that present with a question of attentional functioning, given that the subtests that have been found to measure attention on the WRAML are not even administered.

\section{Gordon Diagnostic System (GDS). The Gordon Diagnostic System (GDS; Gordon,} 1983) provides a measure of subjects' behavior related to sustained attention and inhibition of responses. The system uses an electronic apparatus: a self-contained, single-component microcomputer-driven device measuring 11.5 x 11.5 x 12 inches (Gordon \& Mettelman, 1987, 
1988). The front panel of the portable instrument includes a blue response button measuring approximately 2 inches in diameter, a three-column digital display (red lighting), a correct response indicator light (red), and a "game-over" light (green). The rear panel, inaccessible to the subject, includes a three-column display similar to the one on the front, as well as the knobs and switches for setting task parameters and recording data at the end of each task (Post, Burko, \& Gordon, 1990). The GDS can be used to administer eleven different tasks; only the three most commonly employed tasks were used in the present study, and are thus described here.

The Delay Task of the GDS (Gordon, 1983; Gordon \& Mettelman, 1987) measures the subject's ability to control impulsive responding, through the identification and maintenance of an effective rate of responding in a self-paced activity. The subject is instructed to earn as many "points" as possible by pressing the blue button on the apparatus; the objective of the task is to independently determine the appropriate delay interval between button presses. Responding before the completion of the interval results in the time period being reset; responding at or after the completion of the interval results in the correct response indicator light coming on (for approximately 200 milliseconds) and a point being earned. The delay interval is fixed at six seconds; total time for this subtest is eight minutes. Thus, 80 responses at a 6 -second rate (20 responses in each of four 2-minute blocks) is the maximal number of possible correct responses. Waiting "too long" between responses (e.g., 7 or more seconds) still results in activation of the correct response light, but impedes the subject's ability to earn "points".

The Delay Task yields data on total number of responses, number of correct responses, efficiency of responding, variability in responding across the four blocks of 20 potential responses, and slope of the learning curve. Error analysis data are also provided in terms of number of seconds between responses. For the Delay Task, the efficiency data for each of the 
four response blocks, as well as the total efficiency ratio, were utilized in the present data analysis. This type of data set has been used in other studies (e.g., Aylward et al., 1990; Gordon et al., 1989) and is suggested to be the most critical indicator of impulsivity as measured by this instrument (Gordon \& Mettelman, 1988).

The Vigilance and Distractibility Tasks of the GDS (Gordon, 1983; Gordon \& Mettelman, 1987) measure the subject's ability to focus on and maintain attention to a visual task in the absence of feedback or reinforcement regarding performance. For both tasks, the child is instructed to attend to an "alerting" stimulus (i.e., the numeral '1') on the visual screen, and then to press the button in response to the immediate, subsequent presentation of the "target" stimulus (i.e., the numeral '9'). On the Vigilance Task, numerals (1 through 9) are presented one at a time in the center position of the three-column screen, for 200 milliseconds, with an inter-presentation time of 800 milliseconds (for a total trial time of one second). On the Distractibility Task, numerals are presented at the same rate, though randomly, one at a time, in the three different columns. However, the task demands do not change. That is, the requirement continues to be that the child should respond after observing a '1' followed by a '9' in the center position only. Thus, attention is measured in the presence of additional distractors. Total number of possible correct responses is 45 for both tasks (15 responses in each of three 3-minute blocks); total time for each task is nine minutes.

The Vigilance and Distractibility Tasks yield data on total number of correct responses, numbers of omission and commission errors, and variability in responding across the three blocks of 15 potential responses. Error analysis data, reflecting type of error (target-related or random), and response latencies are also provided. For the purposes of the present study, data regarding total raw numbers of correct responses and commission errors for the Vigilance and 
Distractibility tasks were used in the statistical analyses. Again, this information has been employed in other studies (e.g., Aylward et al., 1990; Gordon et al., 1989) and is suggested to be the most critical indicators of sustained attention as measured by this instrument (Gordon \& Mettelman, 1988).

Technical aspects of the GDS. Initial standardization studies for the GDS were conducted on only the three tasks used in the present study (Gordon \& Mettelman, 1988). The standardization sample consisted of over 1250 males and females between the ages of 4 and 16 years, inclusive, from two mid-sized cities, one in upstate New York (91\% of the participants) and the other in Virginia (9\% of the participants). The full range of socioeconomic status levels was represented in the sample, using the Hollingshead Index (Hollingshead, 1956; in Gordon \& Mettelman, 1987) regarding the primary caretaker's income. Selection criteria for the children in the sample included no history of attentional, behavioral, nor learning problems; intellectual functioning above the mentally impaired range (i.e., IQ > 70); and no significant medical, particularly neurological, problems (Gordon \& Mettelman, 1988). Given findings of significant differences in understanding of tasks and overall task performance between the group of 4-5 year olds versus 6-16 year olds, psychometric data were reported separately for the two groups (Gordon \& Mettelman, 1988). (It should also be noted that, subsequent to the standardization study, variations on the delay and vigilance tasks were developed for preschoolers, aged 3-5 years, as well as variations for all the tasks for adults over the age of 16 years.) Only information for the 6-16 years age group is provided here.

Tests of internal validity showed that intra-task correlations were high among the various scores within a task, but that intercorrelations between the Delay versus Vigilance and Distractibility tasks were not strong. This suggests that the two types of tasks indeed assess 
different aspects of performance (Gordon \& Mettelman, 1988). There was a moderate correlation between the latter two tasks, which would be expected. Test-retest reliability coefficients over a 30-45 day interval ranged from .60 to .85 across the seven scores reported (i.e., efficiency ratio, total responses, and total correct for the Delay task; total correct and total commissions for the Vigilance and Distractibility tasks). After one year, the consistency between obtained scores ranged from .52 to .56 for the Delay task, and .68 to .94 for the Vigilance task. No follow-up data were provided for the Distractibility task (Gordon \& Mettelman, 1988).

Additional standardization data were collected on a sample of approximately 400 Puerto Rican children, aged 6 to 16 years (Bauermeister, Berrios, Jimenez, Acevedo, \& Gordon, 1990). Overall findings indicated that the United States sample had achieved statistically but only moderately higher efficiency ratio scores on the Delay task, as well as moderately higher Total Correct scores and lower numbers of commission errors on the Vigilance task than the Puerto Rican sample. However, the differences between groups was only observed for the younger age groups (6-9 year olds). It is important to be aware that the two sample groups were not matched for socioeconomic status, because of the incompatibility of the SES-assessment instruments available for use. With that limitation noted, these results provided some cross-cultural confirmation of the standardization data for the GDS.

Other neuropsychological measures. The Knox Cube Test (Stone \& Wright, 1981) provides a nonverbal measure of attention span and immediate memory in children and adults. The history of the Knox Cube Test dates back to 1914, when Howard Knox developed the materials and procedure as one part of a test battery for evaluating cognitive functioning among foreign-speaking immigrants arriving at Ellis Island (Knox, 1914, in Stone \& Wright, 1981). 
Several other versions of this test have been developed and implemented over the past 80 years, including Robert Yerkes' (1921, in Stone \& Wright, 1981) use of the procedure in the test battery designed for the U. S. Army, to assess the "mental fitness" of soldiers during World War I. The current adaptation of the test (Stone \& Wright, 1981) was developed in an effort to standardize the materials and the number, length, and composition of the tapping series. In the present version, the authors attempted to demonstrate the internal validity of the measure by constructing an item bank (Rasch, 1960, in Stone \& Wright, 1981) that calibrated all items employed on the various versions of the test along one common scale of cognitive ability, which is described as attention span and immediate memory. Sample size for the current standardization and calibration techniques involved over 450 individuals between the ages of 2 to 69 years.

Test materials for the current version of the Knox Cube Test consist of four black oneinch cubes, placed two inches apart, and attached to a natural-finish wood base measuring 10 inches by 1 1/2 inches. A fifth, separate black cube is used to tap the attached blocks in a given sequence. When the strip of four blocks is placed on the table in front of the individual, the examiner can identify the cubes to him- or herself (the subject is not informed of this) as '1', '2', '3', and '4' from the examiner's right to left. Each item of the test involves a different tapping series (e.g., the examiner's score sheet indicates a sequence such as 1-4-3-2); the items systematically increase in length (i.e., number of taps) and complexity (i.e., distance in blocks traversed and number of changes in direction of movement). The progression of difficulty involves two items at a length of two taps, four items each at three and four taps, five items each at five through seven taps, and one item at eight taps.

The Junior Test Form of the Knox is designed for use with children between the ages of 2 through 8 years; it begins with sequences of two non-crossing taps (e.g., 1-3; 1-4). The Senior 
Test Form (ages 9 years and above) was used for scoring all of the subjects' performances in the present study; it begins with three non-crossing taps (e.g., 1-2-3; 4-2-1). However, if an error occurred on the first or second items of the Senior Test, the examiner administered the first four items of the Junior Test, which are the only items that are different from the Senior Test. This procedure was necessitated for clinical utility of the findings, given that the clinical groups of subjects were actual referred patients seen through the pediatric Neuropsychology Service. Only the scores from the Senior Test Form were used in the data analysis for the current study though, regardless of whether the subject missed any of the first four items from the Junior Form.

Instructions for the Knox Cube Test simply involve the command "Watch", followed by the demonstration of the sequence and then the direction "Do what I did." The tapping block is then handed to the subject. Two practice items are provided at the beginning of the test, to help the subject become familiar with the requirements of the task. Testing continues until the individual demonstrates five consecutive errors in repeating the sequence of taps, or completes all 22 items. Total time for test administration is typically 5 to 15 minutes.

In terms of scoring, one point is given for each correct tapping sequence. Overall raw score is converted to an age-normed score, within a range of $11 / 2$ years above and below the obtained score. The other information pertinent to the individual's performance involves consistency in responding: number of "surprise misses" (incorrect sequences) that occurred below the overall score range, and number of "surprise hits" (correct sequences) that occurred above the overall score range. Raw scores for overall hits, and for surprise hits and misses, were included in the data analysis for the present study.

The Digit Span subtest (a supplementary Verbal Scale subtest) of the WISC-R (Wechsler, 1974) and the WISC-III (Wechsler, 1991) has also been shown to measure children's span of 
attention and immediate memory, as well as mental manipulations of information (Kaufman, 1979). On the WISC-III, this subtest is also represented on the Freedom from Distractibility factor, which reportedly provides a measure of attentional functioning. The Digits Forward portion of the task requires the child to repeat number sequences verbatim, presented orally by the examiner. Sequences are to be repeated in reverse order for the Digits Backward portion, which requires cognitive "holding" and mental manipulations of the information for appropriate responding.

Sequences of numbers involve only single digits (i.e., 1-9 inclusive), and span the range from two to eight units for Digits Forward (three to eight for the WISC-R) and two to seven units for Digits Backward. [It should be noted that for subjects who had been administered the WISC$\mathrm{R}$, credit was assumed for two-unit trials, in order to make raw scores equivalent across all subjects.] Two trials are presented at each span length. One point is given for each correctly repeated sequence. The individual must fail both trials at a given span length, or complete all pairs of sequences, before testing is discontinued on each section. The scores from the two sections (Digits Forward and Backward) are added together to determine the overall raw score for the subtest. The raw score is converted to a scaled score based on age level norms $(\mathrm{mean}=$ 10, standard deviation $=2$ ). Average time for administration of this subtest ranges from 5 to 15 minutes.

Standardization of the WISC-III, for children ages 6-16 years inclusive, was comprehensive and appropriate (Sattler, 1992). Psychometric properties of the WISC-III were also adequate; data regarding the Digit Span subtest in particular are summarized as follows (as presented in the WISC-III Administration Manual; Wechsler, 1991): split-half reliability coefficient was .85 , and test-retest reliability (intervals ranging from 12 to 63 days) was .73 . 
Correlation between Digit Span performance on the WISC-R versus the WISC-III was .71, with scoring typically being lower on the WISC-III (Wechsler, 1991). Standardized information regarding cumulative percentages for correct performance across the progressive sequences of numbers is provided in the WISC-III Manual, as a basis for interpreting discrepancies between scores on the Digits Forward versus Backward portions of the subtest.

In terms of internal validity, the Digit Span subtest loads on the factor designated as Freedom from Distractibility, suggesting that it measures focus and span of attention to verballypresented information. In fact, this subtest was not highly correlated with the verbal problemsolving factor (Wechsler, 1991). Further evidence suggesting an attentional component for this subtest was indicated in a clinical sample: in a group of 68 children with a diagnosis of Attention-Deficit Hyperactivity Disorder, one of the two lowest mean subtest scores was for Digit Span (8.2) (Wechsler, 1991).

\section{Procedure}

All assessments were conducted in testing areas provided by the Department of Behavioral Medicine and Psychiatry at West Virginia University. Sessions were individualized, with only one subject and one evaluator present in the testing room. Tests were administered by trained psychometrists or neuropsychology graduate students, interns, or post-doctoral fellows under the supervision of a licensed clinical neuropsychologist, or by the latter individual. Thus, in some situations, testing may have been observed by the supervisor through a one-way mirror. Each of the tests used in the present study were administered and scored in its standardized format, in an effort to achieve integrity of the testing procedures. However, one modification was made on the WRAML: Story Recognition was administered on the second story (i.e., the fishing story), as well as the third story (i.e., the job story), at the end of the standardized 
administration of the full test. [This additional information was not included in the present analysis, however.] Reliability for accuracy of scoring was assessed through a review of all test protocols for the clinical subjects, by the principal investigator of the study. Any aberrations noted in test administration or scoring automatically excluded a case from being used in this study; this strategy was implemented to help control for problems that might have arisen as a result of using archival data.

Average testing time for the targeted measures was approximately $13 / 4$ - 2 1/4 hours; however, many of the subjects in the clinical sample participated in longer sessions due to the administration of a more extensive neuropsychological battery. Breaks in the testing session(s) were provided as needed, based on the examiner's observation of the subject's behavior (e.g., increased fidgetiness, complaints of being tired, etc.), rather than on the basis of increases in performance errors. Most of the clinical subjects completed the targeted measures within a single day's test session (three of the TBI subjects required two days of testing, and one of the LD/ADHD subjects required two days of testing due to the inclusion of a full psychoeducational assessment). Given the inclusion of the brief intellectual assessment for the control subjects, total testing time for that group was approximately 2 - $21 / 2$ hours.

The order of presentation of the tests was completely counterbalanced across control subjects. One-half of the control group received the neuropsychological measures (WRAML, Knox, Digit Span, GDS) first, and the other half began testing with the IQ measure. Further, one-half of the control group subjects received the GDS prior to the WRAML, with the reverse order of those two tests administered for the other half of the control group. The counterbalancing factor was impossible to control for the clinical subjects, given that archival 
data were used. No documentation of order of test administration had been provided in the records.

\section{$\underline{\text { Data Analysis }}$}

Analysis of the data consisted of two procedures. First, a canonical correlation analysis was conducted to determine the relations between the scores on the GDS tasks as one set of variables, and the scores on the WRAML subtests, Knox Cube Test, and Digit Span subtest (of the WISC-III) as the second set of variables. Follow-up Pearson product-moment correlations were conducted for the variables found to be significant through the canonical analysis. These statistical procedures were selected to address Hypothesis I, which asserted that performance on the GDS would likely be correlated with aspects of the WRAML and other tasks that purportedly measure attentional components. Nine variables from the GDS were entered into the equation: efficiency ratio for each of four response blocks, and total efficiency ratio from the Delay task; and total correct responses and total commission errors from both the Vigilance and Distractibility tasks. Twenty-one variables were entered into the second part of the equation: scores from each of the fourteen WRAML subtests (including the Delay and Story Recognition tasks), overall WRAML GMI score, overall raw score on the Knox Cube Test plus Surprise Hits and Misses, and scaled score on the Digit Span subtest plus scores of Forward and Backward series. Raw scores were converted to $\underline{\mathrm{Z}}$-scores on several variables that were not scaled according to age-level norms (i.e., Delay tasks and Story Recognition task of the WRAML, and Forward and Backward series of the Digit Span subtest).

Variables from each data set that contributed significantly to the canonical correlation were then entered into a direct discriminant function analysis, to determine the significance of the contribution of each of those test variables to the appropriate classification of subjects into 
specific groups. The three groups of clinical subjects included (a) children with a diagnosis of ADHD only, (b) children with dual diagnoses of ADHD and learning disorder (LD), and (c) children with a history of traumatic brain injury (TBI). The control subjects constituted a fourth group. The goal of this analysis was to address Hypothesis II, which predicted that specific performance profiles would discriminate and separate the four groups. 
All statistical analyses were conducted through SPSS for Windows, Version 7.5.2 (SPSS Inc., 1997). Table 4 provides a descriptive summary of the mean scores and standard deviations for each subject group on the GDS, WRAML, Knox Cube Test, and Digit Span subtest. These calculations were used as a basis for computing ANOVAs to determine significant differences between group means on each of the variables. These results provided an initial way of assessing group means and differences across the variables; this data also served as a basis for later post-hoc comparisons (see below). Table 5 reveals that scores on the GDS commission errors (for both Vigilance and Distractibility tasks), Digit Span backward series, Knox Cube Test overall hits, WRAML GMI, and several WRAML subtests (Design Memory, Finger Windows, Sound Symbol and Delay, Verbal Learning and Delay, and Visual Learning Delay) were significantly different across groups $(\mathrm{p}<.05)$. Further analyses, as described below, provide additional information about how the specific groups were separated on these variables. Correlational Analyses

Canonical correlation was performed to assess the relation between the items on the GDS (Set 1), which served as the dependent variable, and the items on the WRAML, Digit Span subtest, and Knox Cube Test (Set 2), which served as the independent variable. The goal of canonical correlation is to analyze the relation between two sets of multiple variables. The analysis redistributes the variance in the original variables into a few pairs of canonical variates, each pair defined by linear combinations of independent and dependent variables and capturing a large share of unique variance. The SPSS system (Version 7.5.2, SPSS Inc., 1997) used a MANOVA program to compute the canonical correlation (see Tabachnick \& Fidell, 1996). 
Several procedures were conducted to ensure that the data sets met statistical assumptions. No univariate outliers (cases with an extreme value on one variable) were identified through casewise analysis of the data; no multivariate outliers (cases with an unusual combination of scores on two or more variables) were identified through Mahalanobis test procedures (computation of the distance of a case and its combined scores from the centroid of the remaining cases; the centroid is created by the means of all the variables). However, four cases were found to have missing data and thus were deleted from the canonical analysis (one LD/ADHD subject, and three TBI subjects). For the 96 cases used in the analysis, evaluation of assumptions of linearity, normality, multicollinearity, and homogeneity of variance-covariance matrices revealed no threat to multivariate analysis. The only exception was that WRAML GMI was essentially a redundant variable, reflecting the combination of nine subtest scores on the WRAML. Thus it violated the requirement for singularity in the data.

Of the nine canonical variates generated in the analysis (equivalent to the number of variables in the smaller data set, Set 1), only the first one was significant. As indicated in Table 6, the first canonical correlation was .736 and contributed $31 \%$ of the variance in the solution. The canonical correlation is interpreted as a typical Pearson product-moment correlation coefficient; the squared canonical correlation would indicate the overlapping variance between the two sets of variables (in this case $\underline{r}^{2}=.542$ ). With all nine canonical variates included in the dimension reduction analysis, $\underline{F}=1.28, \underline{p}<.05$. The remaining canonical correlations were not significant $(\mathrm{p}<.05)$. Thus, the first pair of canonical variates accounted for the significant relations between the two variable sets.

Table 7 presents data for the first pair of canonical variates. Correlational information between each variable and the significant canonical function is indicated, as well as percent of 
variance and redundancies for each variable set. A cutoff correlation of .30 (9\% of variance) was used to determine significance of contribution for each variable, as recommended by Tabachnick \& Fidell (1996).

For the significant canonical variate, the GDS variables of Total Correct on the Vigilance task (.69), and Total Commissions on the Vigilance and Distractibility tasks (-.59 and -.40, respectively), were associated with overall hits on the Knox Cube test (.52), the WRAML GMI (.47), WRAML Design Memory subtest (.52), WRAML Number/Letter Memory subtest (.43), WRAML Verbal Learning task (.36), and WRAML Sound Symbol Delay task (.33). Total percent of variance and total redundancy indices suggest that this pair of canonical variates was only minimally related, when considering the amount of the dependent variable variance accounted for or shared with the independent variable. The percent of shared variance in the dependent variables that contribute to the dependent canonical variate is only $12.9 \%$ (calculated by squaring each of the dependent variable loadings indicated in Table 7, and averaging the results). The Redundancy Index (RI) serves as a measure of the ability of the set of independent variables, taken as a set, to explain the variation in the dependent variable items, taken one at a time (Hair, Anderson, Tatham, \& Black, 1998). Thus the RI is useful in sorting out the shared variance that the two sets of variables contribute to the canonical function. The importance of calculating the redundancy measure of shared variance is that the finding of a relatively strong canonical correlation obtained for a significant canonical variate may be misleading. This is because the canonical correlation represents variance shared by the linear composites of the dependent and independent variable sets, not the variance contributed by each set of variables (Hair et al., 1998). For the present analysis, the RI reveals that the Set 2 variables, which served as the independent variable in this analysis, explained $7 \%$ of the variance 
contributed by the GDS scores (calculated by multiplying the shared variance of the dependent variable by the squared canonical correlation). These findings suggest that the two variable sets have low shared variance, and limited redundancy when compared to the canonical root. That the variable sets were rather independent of each other was expected given the differences in what the variables measure. Some correlations were anticipated however, particularly between the variables in each set that measure attention. This question was examined further in follow-up correlational analyses.

Additional correlational information was obtained through follow-up Pearson productmoment analyses. It should be noted that this information was only calculated for exploratory and descriptive purposes, thus the significance level was not made more stringent than $p<.05$. Table 8 presents the full correlational table, though only the GDS variables that contributed to the significant canonical function are discussed here, in relation to the Set 2 variables. Total Commissions on the Vigilance task were moderately and negatively correlated with Digit Span backwards, WRAML Design Memory, WRAML General Memory Index, WRAML Number/Letter Memory, WRAML Sentence Memory, and WRAML Sound Symbol Memory and Delay tasks. Total Correct on the Vigilance task was moderately and positively correlated with the same Set 2 subtests, with the exception of WRAML Design Memory and WRAML Sound Symbol Memory. However, this Vigilance score was also correlated with overall Digit Span subtest score, all Knox Cube test scores, WRAML Finger Windows, WRAML Verbal Learning, and WRAML Story Recognition subtests. Total Commissions on the Distractibility task were moderately and negatively correlated with only the WRAML Design Memory subtest. Taken together, these findings help to elucidate how certain variables in each of the data sets were related to each other. The follow-up correlations indicate that the two measures of the 
GDS Vigilance Task were moderately correlated with other attention-related test variables. The Total Correct measure of the GDS Vigilance Task was positively correlated with all of the variables that have been shown to measure attention: Digit Span (particularly reverse series), Knox Cube Test, and WRAML Finger Windows, Number/Letter, and Sentence Memory subtests. Total Commission errors on the Vigilance task were significantly but negatively correlated with many of those same subtests. Total Correct score on the Vigilance task also appeared to be correlated with some of the verbally-based WRAML subtests (e.g., Verbal Learning and Story Recognition), while the Vigilance Total Commission scores were correlated with visually-based WRAML subtests that involve configural and spatial memory (e.g., Design Memory and Sound Symbol). The fact that many of these correlations did not show up as significant in the canonical correlation analysis suggests that the linear combinations of these variables did not share a large amount of variance. In fact, despite their significance, the low to moderate Pearson product-moment correlations suggest quite a bit of independence and low redundancy across the variables, which was borne out in the canonical analysis.

One other finding from Table 8 is interesting to note: the profile of correlations for the Total Correct scores on the Vigilance task were dissimilar to the Total Correct scores for the Distractibility task. This suggests that those two tasks may measure different attentional and response factors. Further support for this argument was that the Total Commission scores for the Distractibility task were only correlated with the WRAML Design Memory subtest. Finally, it is important to note that Total Efficiency Ratio on the Delay task, and the efficiency blocks, were correlated with very few of the Set 2 variables, suggesting that this task of impulsivity indeed did not measure sustained attention or memory very well. 
A final descriptive and exploratory analysis was conducted with the variables from Set 2 . This analysis was undertaken to confirm the findings of previous studies which supported specific patterns of relations among certain WRAML subtests, and with other measures of attention (Haut, Haut, \& Franzen, 1992; Sheslow \& Adams, 1990; Stone, 1990). As shown in Table 9, results for the present sample of children, taken as a whole, supported significant correlations among the variables typically thought to be related to attention. Correlations at $\mathrm{p}<.01$ were apparent between Digit Span, Knox Cube Test overall hits, and WRAML Finger Windows, Number/Letter, and Sentence Memory subtests. Further scrutiny of the components of the Digit Span subtest and the Knox Cube Test revealed that Digits Backward was correlated more significantly with the WRAML Finger Windows subtest $(\underline{r}=.430, \underline{p}<.001)$, while Digits Forward was correlated more significantly with the WRAML Number/Letter subtest $(\underline{\mathrm{r}}=.522$, $\mathrm{p}<.001)$. The Surprise Hits score on the Knox Cube Test lent nothing significant to the analysis, and the Surprise Misses score correlated minimally with only the WRAML Number/Letter and Picture Memory subtests. Sentence Memory, another WRAML subtest that has been suggested to reflect an attentional component, was rather strongly correlated with the WRAML Number/Letter subtest $(\underline{\mathrm{r}}=.58, \underline{\mathrm{p}<.001)}$, but only minimally correlated with Digit Span $(\underline{\mathrm{r}}=.26$, $\mathrm{p}=.010)$, and not significantly correlated with WRAML Finger Windows $(\underline{\mathrm{r}}=.18, \underline{\mathrm{p}}=.0762)$.

Another finding of interest was that Digits Backward and Knox Cube Test overall hits were moderately correlated with a number of other WRAML subtests, suggesting that other processes beyond immediate recall may be measured by those tasks.

Among the WRAML measures, the overall GMI was positively and strongly correlated with all subtests and delay tasks $(\mathrm{p}<.001)$. These findings suggest internal consistency for the subtests as measures of overall memory and learning. Among the subtests, Design Memory was 
most strongly correlated with all of the learning subtests and delay tasks $(\mathrm{p}<.001)$. Finger Windows was moderately correlated with the Visual Learning subtest and Delay task, while Number/Letter was strongly to moderately associated with Sentence Memory and Story Memory Delay. These correlations make sense in terms of the visual and verbal attention and memory components, respectively. Picture Memory was moderately correlated ( $\mathrm{p}<.001)$ with Story Memory Delay only, which suggests a contextual component to those tasks. Sound Symbol memory and Delay tasks were moderately associated with a number of WRAML subtests, suggesting that those items tap a variety of attentional and recall abilities.

\section{Direct Discriminant Function Analysis}

A direct discriminant function analysis (SPSS Version 7.5.2, SPSS Inc., 1997) was conducted to explore the differences between the clinical and Control groups, based on groupings of the variables that contributed to the significant canonical function. The goal of discriminant analysis is to predict group membership from a set of predictors. In the present analysis, the predictor variables, taken from the canonical correlation, served as independent variables, and the four groups (ADHD, LD/ADHD, TBI, and Control) served as dependent variables. The primary question was whether appropriate group membership could be predicted from the subjects' performance on the predictor variables, to accomplish group assignment at a better than chance level.

Three of the nine variables that contributed significantly to the canonical function were from the GDS: Total Commissions for the Vigilance and Distractibility tasks, and Total Correct for the Vigilance task. The remaining six variables were from Set 2: overall hits on the Knox Cube Test, WRAML GMI, WRAML Design Memory, WRAML Number/Letter subtest, 
WRAML Sound Symbol Delay task, and WRAML Verbal Learning subtest. These nine variables were entered as predictors of membership in the four subject groups.

Of the original 100 cases, none had out-of-range codes. However, two cases were dropped from the analysis due to missing data. Both cases were from the TBI group: one subject had no GDS data; the other subject was unable to complete the Finger Windows, Number/Letter, and Verbal Learning subtests of the WRAML (thus producing no WRAML GMI score). [The other two cases that had to be dropped from the canonical analysis involved missing Digit Span data; since that subtest was not one of the predictor variables, those two cases were included in the discriminant analysis.]

Table 10 indicates that three discriminant functions were calculated, with a combined $\underline{x}^{2}(27)=65.46, \underline{p}<.001$. After removal of the first function, the second function still indicated a strong association between groups and predictors $\left(\underline{x}^{2}(16)=28.51, \underline{p}<.05\right)$. The third function was not significant. The first discriminant function accounted for approximately $60 \%$ of the between-group variability; the second discriminant function accounted for an additional $23 \%$ of the variability.

The structure matrix for the pattern of correlations between functions and predictors, as seen in Table 11, indicates the predictor variables that contributed significantly to each function. This information is essential for interpreting each of the functions. Loadings in excess of .33 (contributing $10 \%$ of the overlapping variance) were considered to be significant, as suggested by Tabachnick and Fidell (1996). Thus, the predictors that contributed significantly to the first

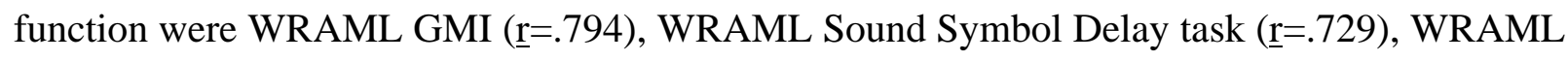
Design Memory ( $\underline{\mathrm{r}}=.570)$, Knox Cube overall hits ( $\underline{\mathrm{r}}=.537)$, GDS Vigilance Total Commissions $(\underline{\mathrm{r}}=-.451)$, WRAML Verbal Learning $(\underline{\mathrm{r}}=.377)$, and GDS Distractibility Total Commissions $(\underline{\mathrm{r}}=$ 
-.329). Predictors that contributed to the second function included Knox Cube overall hits $(\underline{\mathrm{r}}=.458)$ and WRAML Design Memory ( $\underline{\mathrm{r}}=.411)$. WRAML Number/Letter contributed slightly less than $10 \%$ of the variance to the first discriminant function ( $\underline{r}=.308)$, and GDS Vigilance Total Correct contributed significantly to the third discriminant function only, which was overall not helpful in separating the groups. It is interesting to note that in initial univariate $\mathrm{F}$ tests, all of the predictors except GDS Vigilance Total Correct and WRAML Number/Letter were significantly different between groups, which is consistent with these discriminative results (see Table 4).

These findings suggest that the first function could be interpreted as a general working memory factor, due to the fact that the predictors that loaded on that function were neither purely attentional tasks nor "simple" memory tasks. That is, all of the subtests involved a requirement to hold a certain amount of information in memory and work with it prior to responding, and in some cases deal with a rather high level of intervening information as well (e.g., for the Sound Symbol Delay task). The second function seems to specifically be related to a nonverbal working memory factor, given the task requirements to observe a stimulus set, and then reproduce it exactly with no verbal input or responses involved. Further analysis of this data is presented in the Discussion section below.

Information regarding the discriminant functions at the group means, or centroids, is presented in Table 12. Figure 1 presents a graphic display of that data, which illustrates that the first function, potentially a general working memory factor, separated the Control group from the LD/ADHD group. The second function, potentially a nonverbal, or cross-modal, working memory component, further contributed to the separation of the TBI and ADHD groups from the 
Control group. Thus, both discriminant functions were required to effectively separate the three clinical groups from the Control group.

Post-hoc comparisons were conducted with the significant discriminant variables, to further examine how groups differed on each measure. Table 13 presents the follow-up Scheffe tests; within multivariate analyses, this procedure has been suggested to be the most conservative and flexible with respect to Type I error (Hair et al., 1998; Tabachnick \& Fidell, 1996). Results revealed that the LD/ADHD group differed significantly from the Control group on every variable. These findings are consistent with the first discriminant function. The second function predicted separation of the other two clinical groups from the Control group; the post-hoc analyses revealed that those differences occurred on the Knox Cube Test overall hits, and on the WRAML GMI and Design Memory subtest. Thus it appears that the performance of the $\mathrm{LD} / \mathrm{ADHD}$ group on the predictor variables contributed most to the separation of the groups on the first function. Further, the Knox Cube Test overall hits and WRAML Design Memory subtest served to separate the ADHD and TBI groups from the Control group, on the second discriminant function.

Other post-hoc analyses revealed that scores on the WRAML Sound Symbol Delay subtest were significantly different between the Control and TBI groups, as well as the two ADHD groups. That predictor variable contributed significantly to the first discriminant function only, which suggests that the first function also helped to separate the Control group from the other two clinical groups, and the two ADHD groups from each other. However, more separation occurred between the Control group and the ADHD and TBI groups on the second discriminant function. Finally, it was notable that none of the independent variables were significant in separating the TBI group from either of the ADHD groups in the post-hoc analyses. 
The final step of the direct discriminant analysis was to predict group classification based on performance on the independent variables. Results of the classification procedure indicated that of the total usable sample of 98 subjects, 50 (51\%) were classified correctly, compared to the approximately $25 \%$ of cases that would have been classified correctly by chance alone. Review of the classification matrix by group (see Table 14) reveals that accurate membership assignment ranged from $47.8 \%$ to $56 \%$, with the Control group achieving the highest accuracy rate (14 of 25 subjects (56\%) correctly assigned). The LD/ADHD group had $13(52 \%)$ correctly assigned cases; the ADHD group had 12 cases (48\%) correctly assigned; and the TBI group had 11 of 23 cases $(47.8 \%)$ correctly assigned.

Misclassifications for the TBI group occurred across the ADHD groups, with no cases incorrectly assigned to the Control group. Misclassifications for the ADHD group were fairly evenly split between the other three groups; while inaccurate assignments for the LD/ADHD group were more likely to occur in the TBI and Control groups. Relatively few LD/ADHD cases were misassigned to the ADHD group (2 cases; $8 \%$ ). Misassigned Control cases were predominantly split between the ADHD and TBI groups, with only 2 cases (8\%) incorrectly classified as LD/ADHD. 


\section{Discussion}

\section{Hypothesis I}

The first hypothesis addressed in this investigation predicted significant correlations between measures of attention on the GDS, and subtests on the WRAML thought to have a strong attentional component. Two other well-documented measures of attention were included in the analysis as well: the Knox Cube Test, and Digit Span subtest from the WISC-III and WISC-R. Canonical correlation analysis provided some evidence in support of this hypothesis. Results indicated one significant canonical variate, which revealed an expected association between the GDS Vigilance measures (correct responses and commissions), as well as the Distractibility commission errors, and the WRAML Number/Letter subtest and the Knox Cube Test overall hits. [The WRAML GMI scores, also found to be related to the significant canonical variate, will not be discussed, due to reports of unique variance for each WRAML subtest score, and the redundancy factor for the GMI score as a compilation of nine WRAML subtest scores (Callahan et al., 1993; Gioia, 1998).]

The GDS findings are consistent with previous reports that have indicated the utility of the Vigilance task, in particular, for discriminating individuals with suspected attentional deficits (Breen, 1989; Cohen et al., 1989; Grant et al., 1990). Further, the present study found no significant contribution of the Delay task measures to the canonical variate, which is also consistent with recent research reports of poor discriminability of this measure for ADHD individuals (Grant et al., 1990). Additionally, follow-up correlational analyses did not support significant associations between the Delay task and suspected measures of attention on the WRAML, the Digit Span subtest, or the Knox Cube Test. As Barkley et al. (1988) noted, these findings could be an implication that the Delay task is not sensitive to the aspects of impulsivity, 
or behavioral disinhibition, demonstrated by individuals with ADHD and related disorders. It could also mean, however, that impulsivity as measured by the GDS Delay task is very distinct from attention and memory as measured by the WRAML and other tests. In fact, the Delay task was not even significantly correlated with other GDS tasks that purportedly measure sustained attention, suggesting that it does indeed assess a different cognitive or behavioral function.

The WRAML Number/Letter subtest and the Knox Cube Test have been found in previous studies to be significantly correlated with each other (Haut, Haut, \& Franzen, 1992), and, in factor analyses, to load together on an attention/short-term memory factor (Callahan et al., 1993; Haut, Haut, Callahan, 1992). The finding of their significant contributions to the canonical variate was expected. However, other memory tasks that have also been suggested to measure attention (Digit Span forward and backward series, and WRAML Finger Windows and Sentence Memory subtests) did not contribute unique variance to the canonical variate, which was surprising. Follow-up correlational analyses again provided additional information about those subtests though. All five of the memory tasks expected to correlate with GDS attentional measures were, in fact, moderately associated with Total Correct scores on the GDS Vigilance task. This finding supports the assumption that the immediate/short-term memory tasks do involve a significant attentional component, and, perhaps, that the Vigilance task measures some aspects of memory as well. To perform accurately on all of these tasks, for example, the individual does have to remember, or at least "hold" in working memory, the "rule" for when and how to respond. That the Digit Span series, and the WRAML Finger Windows and Sentence Memory subtests, did not contribute to the single significant canonical function suggests that those tasks did not share a large amount of variance with the overall set of GDS variables. In fact, the moderate correlations between Vigilance Total Correct would not necessarily predict a 
significant amount of shared variance for the canonical analysis. It is also interesting to note that Total Correct on the Distractibility task not only contributed poorly to the canonical variate, but also did not correlate significantly with the memory tasks that have a significant attentional component. These results suggest that the Distractibility task may measure quite different aspects of cognitive functioning relative to the Vigilance task.

Another question that could be pertinent regarding the subtests that were expected to contribute to the canonical correlation but did not (e.g., Digit Span, WRAML Finger Windows and Sentence Memory) was whether those tasks had low reliability scores overall. In fact, the WISC-III administration manual reported that Digit Span was associated with moderate reliability measures overall (Wechsler, 1991). Finger Windows also had some of the lowest reliability scores for the WRAML standardization, though those findings were still in the moderate to high range (Sheslow \& Adams, 1990). However, Sentence Memory actually had some of the highest reliability scores for the WRAML; thus, this argument does not hold across all of the subtests. Other plausible explanations for this unanticipated finding are suggested below.

A second unexpected finding for the canonical correlation was the significant contributions of variance for the WRAML Design Memory, Verbal Learning, and Sound Symbol Delay subtests. Follow-up analyses revealed that Verbal Learning correlated with the Vigilance Total Correct scores, and that Design Memory and the Sound Symbol Learning and Delay tasks were related to the Vigilance Total Commission scores. These findings suggest that perhaps the accuracy component of the Vigilance task involves aspects of verbal mediation and thus verbal working memory. This suggestion could account for the individual's "rule-governed" responses; that is, remembering and acting upon the rule to hit the target button after observing the specified 
numeric sequence. The fact that the Verbal Learning task has multiple trials may also be an important factor in its relation to Vigilance Total Correct, in that the latter task actually involves repeated "trials" as well.

Alternately, the fact that the commission component of the Vigilance task was correlated with the Design Memory and Sound Symbol tasks suggests that the former measure may involve more aspects of configural and spatial attention and memory, which would tend to invoke nonverbal working memory. This cognitive function could account for the individual's ability to hold the "rule" in memory, and to effectively scan and detect specific stimuli from the continuous presentation of numerals. It is also interesting to consider that both the Design Memory and Sound Symbol Delay tasks involve some "wait time": a 10-second delay before being allowed to respond on the former task, and up to a 30-40 minute delay before returning to the Sound Symbol Delay task. The Commission measure of the Vigilance task also involves waiting, as well as requirements to process intervening stimuli. Although no intervening stimuli are presented during the 10-second delay on the Design Memory subtest, it is possible that an individual with behavioral disinhibition problems could become distracted during the delay. It is clear that much intervening material is presented to an individual prior to the final Sound Symbol Delay trial. Thus, the issues of "wait time", distractibility, and delaying gratification may be playing significant roles, related to nonverbal working memory, in an individual's performance across these three subtests.

It could also be suggested that, in relation to Vigilance task commissions, an individual still has to "label" the numerals, and thus there could be a verbal working memory component involved as well. This fits with the factor analytic conceptualizations of Design Memory and the Sound Symbol tasks as representing cross-modal attention and memory functions (Gioia, 1998; 
Haut, Haut, Callahan, et al., 1992). Design Memory, for example, involves "incidental" memory of known information, applied to novel situations. According to Brown's (1975) formulation of memory development, Design Memory would be considered a non-strategic, episodic task, involving minimal mnemonic strategies and limited contextual relations. However, given that the individual typically has learned the labels for the common geometric figures used in the task, it is likely that he or she would use the labels to help memorize and recall the new configurations of symbols (i.e., relying on "incidental" memory). Thus Design Memory and Vigilance Total Commissions tasks seem to involve both visual and verbal working memory components. This suggestion is supported by the fact that Total Commissions on the Distractibility task were also significantly correlated with Design Memory (and no other subtests, in fact).

The Sound Symbol tasks have even more obvious cross-modal elements, in that the individual is required to attend to and memorize both a novel two-dimensional visual design and an associated verbal nonsense label (e.g., a paired-associate task). Again, this activity could be considered an episodic memory task, though with some strategic elements for associating the design and label. In factor analyses, the Sound Symbol tasks have typically loaded on either a general memory factor (Gioia, 1998), or have stood alone, not significantly loading on a verbal, visual, or attentional factor (Donders, 1995). Indeed, in follow-up analyses with the present data set, the Sound Symbol tasks were significantly correlated with every WRAML subtest except Picture Memory, and with Digit Span backward series and Knox Cube overall hits.

Taken together then, it appears that whereas Total Correct on the Vigilance task is more related to attention and verbal working memory, Total Commissions reflect an additional visual attention and nonverbal working memory component (producing more of a cross-modal effect). Although these results of the canonical correlation were somewhat unexpected, interpretation of 
those findings along the working memory dimension, rather than the "traditional" attention versus memory dimension, may lead to a better understanding of what the GDS and WRAML tasks measure. In fact, the interpretations presented above fit well with Barkley's recent conceptualization of ADHD (Barkley, 1997, 1998). Similar to Denckla (1996), Barkley proposed that deficits in behavioral inhibition affect several executive functions in individuals with ADHD, including working memory. Deficits in verbal working memory for the present sample are suggested in the correlations between attention and memory tasks that involve a verbal or verbal-mediation component, and the GDS Vigilance task. The current results appear to provide support for the notion that difficulties with "self-talk" about the problem to be solved, and with consistency in rule-governed behavior, are related to deficits in sustained attention, vigilance, and rote memorization and recall of episodic information. For instance, in the present subject sample, correlations between scores on the Number/Letter, Verbal Learning, and Vigilance Total Correct subtests suggest deficits in labeling information so that it can be appropriately recognized and categorized in comparison to previous experiences.

Similarly, nonverbal working memory appears to be related to difficulties in "holding" information in a cognitive "processing" area, in order to manipulate the information as necessary and to act or respond according to appropriate time frames. The Knox Cube Test is a good example of a task that requires an individual to hold the initially-presented information in "memory", and then to plan the reproduction of the sequenced movements. The Vigilance task also requires the holding of information in a memory or processing area, as in holding the numeral ' 1 ' until the next numeral appears, and then judging quickly if it is a '9'. Managing intervening information is also a task for nonverbal working memory, such as the delays that are required to solve the Design Memory and Sound Symbol Delay tasks. 
Researchers are just beginning to investigate the relation of verbal and nonverbal working memory deficits to the behavioral and cognitive functioning of individuals with attentional difficulties (e.g., Denckla, 1996). These types of cognitive processing problems seem logical to assess in persons with ADHD and similar disorders, due to neuroanatomical evidence of frontal lobe involvement in both types of difficulties. Indeed, the suggestions of Denckla (1996), that ADHD involves a disorder of "intention", and Barkley $(1997,1998)$ that ADHD involves problems with behavioral disinhibition, imply a "core" deficit that is more pervasive than just "sustained attention" or "impulsivity". The current results provide evidence for that broader conceptualization of attentional difficulties, by suggesting significant deficits in working memory and timing related to a wide range of activities and behaviors.

In sum then, the first research question appears to have a "broader" answer than initially anticipated. However, the results do support the utility of administering both "attentional" and "memory" tasks when evaluating individuals with suspected attentional difficulties. Correlations between the two types of measures can provide valuable diagnostic and clinical information. The present data suggest, though, that it is important to consider the integrated roles of attention and memory among the various tasks, rather than interpreting each as separate processes.

A second question related to the current data set was how well the correlated tasks, taken as a group from the significant canonical variate, accurately discriminated individuals with attentional difficulties from typically-developing peers. Further, membership in different subgroups of individuals with attention deficits were predicted to be related to variations in score profiles on the target measures. This second research hypothesis was addressed through a direct discriminant analysis. 
Hypothesis II

In order to determine predictions of group membership, the nine subtests that contributed to the significant canonical variate were submitted to a direct discriminant analysis. Two significant discriminant functions emerged from this analysis: the first function appeared to separate the Control group from the LD/ADHD clinical group, on a general working memory dimension; the second function further separated the ADHD and TBI groups from the Control group, on a nonverbal working memory dimension.

Post-hoc analyses revealed that the LD/ADHD group demonstrated more pervasive difficulties on the predictor variable tasks than the other two clinical groups, relative to the Control group. Although all of the clinical groups were different from the Control group on the Knox Cube overall hits and the WRAML Design Memory subtest, and both the TBI and LD/ADHD groups differed from the Control group on WRAML Sound Symbol Delay, the latter group was uniquely (and significantly) different from the Control group on Total Commissions for both Vigilance and Distractibility, and on WRAML Verbal Learning. These results are consistent with previous investigations that have found the commission errors on the GDS to discriminate between LD and ADHD subjects (Barkley et al., 1990; Robins, 1992, and the WRAML Verbal Learning subtest to discriminate ADHD subjects from normally-functioning controls (Hooper et al., 1992). This information about the LD/ADHD group suggests that cognitive processing beyond "simple" attentional functioning may be involved in activities that have traditionally been thought to measure vigilance in attending (Vigilance and Distractibility Total Commissions). Further, memory tasks may add a significant piece of diagnostic information to the question of attentional difficulties, in terms of tapping the learning problems that tend to occur concomitantly with deficits in organization and behavioral inhibition (Verbal 
Learning). The nature of the separation between the Control group and the LD/ADHD groups could be interpreted as being related to a general working memory function, incorporating both verbal and nonverbal elements. As indicated with the canonical correlation, aspects of verbal and nonverbal working memory appeared to play a significant role in the subjects' performance difficulties on many of the predictor tasks.

In fact, the second function suggested that nonverbal, or perhaps, cross-modal, working memory tasks further separated the Control group from the ADHD and TBI groups. Specifically, it was performances on the Knox Cube Test overall hits and WRAML Design Memory that cumulatively contributed to the separation between the former group and the other two clinical groups. TBI groups with moderate injuries have been found to demonstrate difficulties on tasks that involve visual reproduction, as is required for the Knox Cube and Design Memory tasks (Farmer et al., 1999; Williams \& Haut, 1995). Thus, the finding that these two tests help to separate the TBI and Control groups was not surprising. For the ADHD groups, this finding suggests significant difficulties in using verbal and nonverbal working memory to solve problems that involve non-strategic, episodic information. The more pervasive performance deficits demonstrated by the LD/ADHD group, on other variables, suggests that learning disorders further negatively impact the functional cognitive abilities of children with ADHD. The Sound Symbol Delay task was the one subtest that seemed to separate the two ADHD groups; in fact, the LD/ADHD group performed at a significantly lower level on that task compared to the "pure" ADHD group. The requirement to learn two different types of noncontextual, abstract information was apparently quite taxing for the individuals who suffered both attentional and learning problems. Children with ADHD only did not differ from the Control group on that variable, suggesting that it involves more of a learning than attentional 
factor. The fact that many of the TBI subjects in the present study also demonstrated some learning problems supports this interpretation, given that group's separation from the Control group on Sound Symbol Delay task as well.

An unexpected finding of the discriminant analysis was that Number/Letter Memory and Vigilance Total Correct scores did not contribute significantly to the separation of groups, particularly given that both subtests were correlated with all purported measures of "attention" on the WRAML, Digit Span, and Knox Cube tests. Further, Pearson product-moment correlations indicated a moderate correlation between the two subtests themselves, which suggests that they measure similar cognitive functions, perhaps different from what the significant discriminant variables measured. It could be speculated that Vigilance Total Correct and WRAML Number/Letter do not rely on working memory to the extent that the other subtests do. That is, perhaps those tasks involve fewer mental manipulations or mnemonic strategies than the other tasks, and instead measure "purer" attention and immediate recall with minimal processing demands. In keeping with the earlier discussion of the salience of working memory deficits in individuals with ADHD, these assumptions imply that tasks that measure working memory may be more effective in discriminating children with attention deficits, particularly when learning difficulties are also involved. In fact, the tasks that seemed to involve both a visual and verbal working memory component appeared to be the "best" predictor variables.

In sum, the overall findings related to the two significant discriminant functions were that the LD/ADHD group appeared to be impaired on all the predictor variables, while the other two clinical groups were separable from the Control group on tasks that involved visual-spatial skills, and perhaps visual and verbal working memory components. One reason for the rather unexpected finding that the LD/ADHD group would be more "globally" impaired could be due 
to the inclusion of both verbal and nonverbal LDs in the group. In fact, the group was evenly split between subjects with verbal LDs related to reading and spelling, versus nonverbal LDs related to math and written language. Thus it could be that the various predictor variables effectively "covered" all of the learning problems of the LD/ADHD group. However, another plausible explanation for this finding could be related to the concept of "brain reserve capacity", as suggested by Satz (1993). This hypothesis holds that individuals with one "problem", such as ADHD, might be able to "compensate" for those difficulties because they still have enough residual "brain capacity" to be able to function relatively effectively. However, each individual has a threshold beyond which effective functioning becomes difficult; multiple problems, such as having a comorbid learning disability concomitant with attention deficits, may stretch the limits of that threshold and result in more significant functional weaknesses for the individual. This could be true in the present subject sample for the LD/ADHD group, and also for the third of the TBI group that had documented intellectual or achievement difficulties post-injury. Subjects in the "pure" ADHD group were thought to have only the single functional deficit (i.e.., attentional problems), thus it would be expected that that group would have a reserve of brain capacity, and likely not demonstrate as many functional difficulties. However, it is also important to consider that one-fifth of the ADHD subjects were taking prescribed psychostimulant medication on the day of the evaluation, thus that factor may also have masked some of the deficits that might have been displayed by those particular subjects. The final analysis conducted for this study, that of group assignments based on performance on the predictor variables, provided further information regarding this issue.

The second research hypothesis did predict that the Control group would perform more accurately and efficiently on all attentional and memory tasks than the clinical groups. However, 
it was also predicted that the "pure" ADHD group would perform less well on tests thought to measure attention, and that the LD/ADHD group would have more trouble with the memory tasks. The TBI group was expected to demonstrate deficits on all tasks, and particularly on visual-spatial tasks. Results of the discriminant function analysis indicated pervasive deficits in attention and memory functioning for the LD/ADHD group, with quite a bit of overlap with the TBI group on performance on the predictor variables. The classification procedure reflected these findings, with $20 \%$ of the LD/ADHD group misassigned to the TBI group. This makes sense, given that many individuals with TBIs also demonstrate post-morbid learning difficulties. Specifically, one-third to one-half of the present TBI group were noted to have intellectual and/or achievement scores in the low average to borderline range post-injury, which could be considered a significant learning problem.

In fact, the TBI group suffered the least accurate classification rate, perhaps reflecting the similar clinical presentations of the attentional and learning deficits of each of the other two clinical groups. It is significant to note, however, that the TBI group had no misclassifications in the Control group, which supports previous findings of the long-term impact of even a mild TBI on cognitive and learning abilities (e.g.., Parker, 1994). Questions remain about the nature of the deficits demonstrated by children with TBI though. For example, it is unclear whether the variable sites of structural damage, experienced by individuals with TBI, produce more diffuse and/or global attentional, learning, and memory deficits than children with diagnosable ADHD, for whom frontal pathways are particularly implicated. Further, are the attentional difficulties experienced by children with TBI related to the same functional deficits that Barkley (1997, 1998) and Denckla (1996) proposed for individuals with ADHD? Does TBI in children produce behavioral disinhibition, manifested in deficits in working memory and behavioral timing, or do 
their attentional problems have more to do with perception or "sensory register" deficits? Further research would be required to address those questions.

The misclassifications of the ADHD subjects into the other two clinical groups reflect the fact that the former group did not demonstrate deficits on the attentional measures only. Rather, demonstrated problems across a variety of attentional and memory tasks suggests, perhaps, more pervasive aspects of working memory deficits, as described above. The fact that nine subjects from the two ADHD groups were misclassified into the Control group is also important, in light of the frequent questions about the range of "normalcy" in attentional behaviors, and perhaps the over-diagnosis of ADHD (e.g., Newcorn et al., 1994). This dilemma illustrates the need for further research in this area as well.

Overall, the discriminant analysis resulted in appropriate prediction of group membership for a little over half of the subject population. Within each group, subjects were also classified correctly approximately $50 \%$ of the time. Another way to interpret these data are to think in terms of sensitivity and specificity of the measures (e.g., Reid \& Maag, 1994). The classification data indicate the rate of "true positive" cases for each group; those results suggest that the predictor variables were not particularly sensitive to appropriate discrimination of the four groups. However, the predictors were slightly more effective at detecting "true negatives": cases that were not assigned to a particular group, and did not, in fact, have the clinical diagnosis of that group (e.g., a subject who did not have ADHD, and was not assigned to that group). This measure of specificity for the classification data ranged from $60.2 \%$ for the ADHD group to $65.3 \%$ for the Control group (average $=62.7 \%$ ). Thus, information about sensitivity and specificity of the data do not indicate particularly strong rates of accurate classification, using the predictor variables. The reasons why predicted group membership rates were not more accurate 
are best considered in the context of the limitations of the overall study, which are discussed in the following section.

\section{Limitations of the study and implications for further research}

Several limitations to the interpretation of the present results should be noted. First, the group sizes were small. With only 25 subjects included in each group (and numbers as low as 23 subjects in a group when missing data resulted in cases being dropped from analysis), the validity and generalizability of the obtained results is likely questionable. Further, statistical power could have been affected. Together, these concerns likely impacted the moderate correctional and classification results.

Another threat to statistical power was the inclusion of more test variables than the number of subjects within a group, for the canonical correlation procedure. Tabachnick and Fidell (1996) warned that that statistical analysis, in particular, is sensitive to the variable/sample size ratio. Further, canonical correlation is reportedly sensitive to even minor changes in one set of variables, such as cases with missing data. It might have been possible to estimate data for each of those cases, using procedures suggested by Tabachnick and Fidell (1996). However, those same authors supported the robustness of the discriminant analysis for as few as 20 cases in the smallest group, if only a few predictors were entered into the analysis. In the present study, nine predictors were used, with the smallest of the four groups having 23 cases. Further, discriminant analysis is apparently less sensitive to slightly unequal group sizes.

There were additional sampling issues within the groups, again partly affected by small sample size. First, although the two ADHD groups were diagnostically identified according to the DSM-IV subtypes, the data analysis was not designed to look at each of those smaller groups separately (e.g., inattentive versus impulsive-hyperactive versus combined subtypes). A much 
larger sample size would be required to address the question of whether different ADHD subtypes are associated with particular performance profiles on tests of attention and memory. Second, for the LD/ADHD group, two different methods were used to identify learning disorders. That is, subjects were included in the sample if they had a specific achievement discrepancy relative to IQ scores in the average range, or if they displayed a significant VerbalPerformance IQ split. Again, the data analysis did not account for any potential differences in performance and functioning for the two subgroups of LD subjects, though descriptive interpretations were offered on the basis of types of LDs documented. Additionally, this study included no "pure" LD group for comparison measures, which may have helped to elucidate the differences between the subject groups with learning difficulties and the other clinical groups (e.g., Robins, 1992). Finally, the TBI group was heterogeneous in terms of types of injury, location of suspected brain lesions, length of unconsciousness, and pre- and post-morbid functioning. Further, only one type of information (i.e., brain scans, but no clear information about GCS scores or time of LOC) was available in the medical records of those subjects to attempt to determine the severity of head injury. Thus, it might be difficult to generalize the present findings to other populations of TBI children, particularly those who have sustained severe injuries.

Another issue related to subject classification was the overlapping nature of the clinical characteristics in the three groups. All of the clinical subjects demonstrated problems with attention, which of course was a criterion for inclusion in the study. Further, according to Denckla (1996) and Barkley et al. (1992), most children with ADHD demonstrate some aspects of learning difficulties, even if their psychoeducational profiles do not indicate specific learning disorders. The finding that the Control group in the present study had some overlap with the 
ADHD group again raises the frequent clinical question of the range of "normalcy" in attention and behavioral inhibition in children (e.g., Newcorn et al., 1994). It may be that the assessment measures used in the present investigation were not the most sensitive in terms of detecting specific differences between these very similar groups. Perhaps a broader evaluation battery would provide additional information to support the clinical utility of test performances on the GDS and WRAML. For example, in neuropsychological batteries for individuals with suspected attention problems, researchers have included not only tests of attention and memory, but also tools that measure reaction times, processing speed, visual-motor ability, perceptual-motor skills, and higher-order cognitive skills such as set shifting (e.g., Aylward, 1994; Loge et al., 1990; McMillan et al., 1993; Robins, 1992). A related question for further investigation would be the similarity of the current findings to other CPTs and other memory tests.

A final issue about subject data for the clinical groups is related to the use of archival information. Several problems could be inherent in such data collection methods, such as lack of standardization in the order of test administration, application of various criteria for when to provide subjects with breaks in the sessions, heterogeneity in testing styles among examiners, and virtually no ability to conduct checks of integrity on examiner administration of the various tasks. Steps were taken, as possible, to limit any confounds in the use of archival data, but of course not everything could be controlled retrospectively.

\section{Conclusions}

This research investigation accomplished its goals, which were (1) to compare the performances of children with documented attentional difficulties on tests purported to measure impulsivity, sustained attention, and short- and long-term memory, and (2) to determine whether test profiles on attention and memory measures were clinically useful in discriminating groups of 
children with similar presentations of attentional and learning weaknesses. Though several limitations to the data interpretation must be considered, results suggested that deficits in the executive function elements of verbal and nonverbal working memory, particularly related to episodic attention and memory tasks, provide significant discriminability between children with and without attentional problems. If working memory is thought of as a learning mechanism, then it makes further sense that the children in the present sample who had documented learning disabilities would perform more poorly on tasks that involve working memory processes. The fact that the two other clinical groups also demonstrated performance difficulties on a variety of tasks, particularly in the performance domain, suggests that attention deficits likely have a strong correlation with learning problems. Thus, discrimination among the three clinical groups on the measures administered in this investigation was moderate at best, likely due to the overlapping processes required for many of the tasks.

The present findings extend previous research in this area, which had acknowledged that some purported tests of memory were correlated with measures of attention. The present results are also consistent with recent discussions that have pointed to working memory as a discriminating variable for individuals with ADHD (Denckla, 1996). In particular, these findings support Barkley's $(1997,1998)$ noteworthy conceptualization of ADHD as a disorder of behavioral disinhibition, affecting working memory and other aspects of executive function, and being manifested in timing of responses and reactions. However, the nature of the relations between attention and memory functions in children with ADHD have still not been clearly identified, especially as related to neuropsychological assessments. If tests such as the GDS and the WRAML can be reconsidered as more than "simple" measures of impulsivity, sustained attention, and short- and long-term memory, respectively, their clinical utility in diagnosing and 
planning treatment for children with ADHD and related disorders could likely be enhanced. For example, the GDS Vigilance task may, in fact, be an appropriate measure of behavioral disinhibition, rather than "just" sustained attention. On the other hand, more research about the working memory elements of some of the WRAML subtests will need to be examined, due to some suggestion that none of the WRAML measures involve inferential processing or reconfiguration, which are components of working memory (Phelps, 1996). Of course, for diagnosis and treatment planning, both of these measures should consistently be included as components of a broader-based evaluation battery. One issue that should continue to be investigated along these lines is the aspects of certain attention and memory tasks that make them more difficult to learn, for specific populations of children. A related question is to identify which conditions (environmental, physiological) are correlated with specific performance profiles on tests of attention and memory.

One final issue is whether the present findings lend any new information regarding service delivery to children with attentional difficulties. In diagnostic assessment, new considerations of the interactions of attention and memory, especially along the lines of working memory, could enhance the practitioner's ability to accurately identify both attention deficits and learning difficulties. Further, treatment planning might be more appropriate if efforts are made to include strategies for modifying instructional techniques, so that the child with attention and learning problems can more effectively process and act upon information. Certainly, ADHD children should be thoroughly evaluated regarding learning difficulties related to their problems with behavioral disinhibition, and students with comorbid attentional and learning deficits should be considered for significant educational and therapeutic support regarding their multiple disabilities. Children with a TBI should undergo specific assessment of a variety of functional 
skills, in order to identify individual needs and develop appropriate programming as indicated. It is hoped that this information adds to the existing body of literature and expertise related to the diagnosis and treatment of these clinical groups of children. 


\section{References}

Ackerman, B.P. (1996). Induction of a memory retrieval strategy by young children. Journal of Experimental Child Psychology, 62, 243-271.

Adams, W., Robins, P., Sheslow, D., \& Wilkinson, G. (1992, November). Memory abilities in children with attention deficit hyperactivity disorder. In W. Adams (Chair), Wide

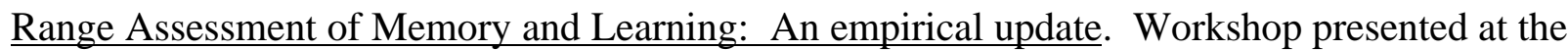
12th Annual Meeting of the National Academy of Neuropsychology, Pittsburgh, PA.

Adams, W., Sheslow, D., Robins, P., Payne, H., \& Wilkinson, G. (1991, August). Memory abilities in children with attention deficit hyperactivity disorder. Paper presented at the 99th Annual Convention of the American Psychological Association, San Francisco, CA.

Adams, W., Sheslow, D., \& Wilkinson, G. (1992, November). Assessment of memory and learning in children: Supplemental norms. Paper presented at the 12th Annual Meeting of the National Academy of Neuropsychology, Pittsburgh, PA.

Aman, C.J., Roberts, R.J., \& Pennington, B.F. (1998). A neuropsychological examination of the underlying deficit in attention deficit hyperactivity disorder: Frontal lobe versus right parietal lobe theories. Developmental Psychology, 34, 956-969.

American Psychiatric Association. (1980). Diagnostic and statistical manual of mental disorders (3rd ed.). Washington, DC: Author.

American Psychiatric Association. (1987). Diagnostic and statistical manual of mental disorders (3rd ed., revised). Washington, DC: Author.

American Psychiatric Association. (1994). Diagnostic and statistical manual of mental disorders (4th ed.). Washington, DC: Author. 
Anastopoulos, A.D., Spisto, M.A., \& Maher, M.C. (1994). The WISC-III Freedom from Distractibility Factor: Its utility in identifying children with attention deficit hyperactivity disorder. Psychological Assessment, 6, 368-371.

Anderson, V.A., Morse, S.A., Klug, G., Catroppa, C., Haritou, F., Rosenfeld, J., \& Pentland, L. (1997). Predicting recovery from head injury in young children: A prospective analysis. Journal of the International Neuropsychological Society, 3, 568-580.

Armstrong, T. (1996). ADD: Does it really exist? Phi Delta Kappan, 77, 424-428.

Atkinson, R., \& Shiffrin, R. (1968). Human memory: A proposed system and its control processes. In K.W. Spence \& J.T. Spence (Eds.), The psychology of learning and motivation (Vol. 2, pp. 90-197). New York: Academic Press.

August, G.J., \& Garfinkel, B.D. (1989). Behavioral and cognitive subtypes of ADHD. Journal of the American Academy of Child and Adolescent Psychiatry, 28, 739-748.

August, G.J., \& Garfinkel, B.D. (1990). Comorbidity of ADHD and reading disability among clinic-referred children. Journal of Abnormal Child Psychology, 18, 29-45.

Aylward, G.P. (1994, February). Measurement of memory and attention in an ADD/LD population. Paper presented at the 22nd Annual Meeting of the International Neuropsychological Society, Cincinnati, $\mathrm{OH}$.

Aylward, G.P., Gioia, G.A., Verhulst, S.J., \& Bell, S. (1994, February). Factor structure of the WRAML in a clinical population. Paper presented at the 22nd Annual Meeting of the International Neuropsychological Society, Cincinnati, OH.

Aylward, G.P., Verhulst, S.J., \& Bell, S. (1990). Individual and combined effects of attention deficits and learning disabilities on computerized ADHD assessment. $\underline{\text { Journal of }}$ Psychoeducational Assessment, 8, 497-508. 
Baddeley, A. (1986). Working memory. New York: Oxford University Press.

Baddeley, A. (1998). Recent developments in working memory. Current Opinion in

Neurobiology, 8, 234-238.

Barkley, R.A. (1988). Attention. In M.G. Tramontana \& S.R. Hooper (Eds.), Assessment issues in child neuropsychology (pp. 145-176). New York: Plenum Press.

Barkley, R.A. (1990). Attention deficit hyperactivity disorder: A handbook for diagnosis and treatment. New York: Guilford Press.

Barkley, R.A. (1991). The ecological validity of the laboratory and analogue assessment methods of ADHD symptoms. Journal of Abnormal Child Psychology, 19, 149-178.

Barkley, R.A. (1997). Behavioral inhibition, sustained attention, and executive functions: Constructing a unifying theory of ADHD. Psychological Bulletin, 121, 65-94.

Barkley, R.A. (1998). Attention deficit hyperactivity disorder: A handbook for diagnosis and treatment (2nd ed.). New York: Guilford Press.

Barkley, R.A., DuPaul, G.J., \& McMurray, M.B. (1990). Comprehensive evaluation of attention deficit disorder with and without hyperactivity as defined by research criteria. $\underline{\text { Journal }}$ of Consulting and Clinical Psychology, 58, 775-789.

Barkley, R.A., DuPaul, G.J., \& McMurray, M.B. (1991). Attention deficit disorder with and without hyperactivity: Clinical response to three dose levels of methylphenidate. Pediatrics, $\underline{87,}$ 519-531.

Barkley, R.A., Fischer, M., Newby, R., \& Breen, M. (1988). Development of a multimethod clinical protocol for assessing stimulant drug responses in ADHD children. $\underline{\text { Journal of }}$ Clinical Child Psychology, 17, 14-24. 
Barkley, R.A., Grodzinsky, G., \& DuPaul, G.J. (1992). Frontal lobe functions in attention deficit disorder with and without hyperactivity: A review and research report. of Abnormal Child Psychology, 20, 163-188.

Bauer, P.J., Hertsgaard, L.A., \& Dow, G.A. (1994). After 8 months have passed: Longterm recall of events by 1- to 2-year-old children. Memory, 2, 353-382.

Bauermeister, J.J., Berrios, V., Jimenez, A.L., Acevedo, L., \& Gordon, M. (1990). Some issues and instruments for the assessment of attention-deficit hyperactivity disorder in Puerto Rican children. Journal of Clinical Child Psychology, 19, 9-16.

Baumgaertel, A., Wolraich, M., \& Dietrich, M. (1995). Comparison of diagnostic criteria for attention deficit disorders in a German elementary school sample. Journal of the American Academy of Child and Adolescent Psychiatry, 34, 629-638.

Berman, S., \& Friedman, D. (1995). The development of selective attention as reflected by event-related brain potentials. Journal of Experimental Child Psychology, 59, 1-31.

Blondis, T.A., Accardo, P.J., \& Snow, J.H. (1989). Measures of attention deficit. Part II: Clinical perspectives and test interpretation. Clinical Pediatrics (Philadelphia), 28, 268-276.

Boller, K., \& Rovee-Collier, C. (1992). Contextual coding and recoding of infants' memories. Journal of Experimental Child Psychology, 53, 1-23.

Bornstein, M.H., \& Sigman, M.D. (1986). Continuity in mental development from infancy. Child Development, 57, 251-274.

Bornstein, R.A. (1983). Construct validity of the Knox Cube Test as a neuropsychological measure. Journal of Clinical Neuropsychology, 5, 105-114. 
Boyd, T.A. (1988). Clinical assessment of memory in children: A developmental framework for practice. In M.G. Tramontana \& S.R. Hooper (Eds.), Assessment issues in child neuropsychology (pp. 177-204). New York: Plenum Press.

Boyer, M.E., Barron, K.L., \& Farrar, M.J. (1994). Three-year-olds remember a novel event from 20 months: Evidence of long-term memory in children? Memory, 2, 417-445.

Branch, M.N. (1977). On the role of "memory" in behavior analysis. Journal of the Experimental Analysis of Behavior, 28, 171-179.

Breen, M.J. (1989). Cognitive and behavioral differences in ADHD boys and girls. Journal of Child Psychology and Psychiatry, 30, 711-716.

Brown, A. (1975). The development of memory: Knowing, knowing about knowing, and knowing how to know. In H.W. Reese (Ed.), Advances in child development and behavior (Vol. 10, pp. 103-152). New York: Academic Press.

Brown, A., Bransford, J., Ferrara, R., \& Campione, J. (1983). Learning, remembering, and understanding. In P. Mussen, J. Flavell, \& E. Markman (Eds.), Handbook of child psychology (Vol. 3, pp. 77-166). New York: Wiley.

Brown, R.T., \& Wynne, M.E. (1984). Attentional characteristics and teacher ratings in hyperactive, reading disabled, and normal boys. Journal of Child Clinical Psychology, 13, 3843.

Bruce, D.A. (1995). Pathophysiological responses of the child's brain following trauma. In S.H. Broman \& M.E. Michel (Eds.), Traumatic head injury in children (pp. 40-51).

Burg, J.S., Burright, R.G., \& Donovick, P.J. (1995). Performance data for traumatic brain-injured subjects on the Gordon Diagnostic System (GDS) tests of attention. Brain Injury, 9, 395-403. 
Burtis, P.J. (1982). Capacity increase and chunking in the development of short-term memory. Journal of Experimental Child Psychology, 34, 387-413.

Burton, D.B., Mittenberg, W., \& Burton, C.A. (1993, February). A structural equation analysis of the Wide Range Assessment of Memory and Learning in the standardization sample. Paper presented at the 21st Annual Meeting of the International Neuropsychological Society, Galveston, TX.

Callahan, T.S., Haut, J.S., Haut, M.W., \& Franzen, M.D. (1993, August). Confirmatory factor analysis of the WRAML. Paper presented at the 101st Annual Convention of the American Psychological Association, Toronto, Canada.

Cantwell, D.P. (1996). Attention deficit disorder: A review of the past 10 years. Journal of the American Academy of Child and Adolescent Psychiatry, 35, 978-987.

Carlson, G.A., \& Rapport, M.D. (1989). Diagnostic classification issues in attentiondeficit hyperactivity disorder. Psychiatric Annals, 19, 576-583.

Carte, E.T., Nigg, J.T., \& Hinshaw, S.P. (1996). Neuropsychological functioning, motor speed, and language processing in boys with and without ADHD. Journal of Abnormal Child Psychology, 24, 481-498.

Carter, C.S., Krener, P., Chaderjian, M., Northcutt, C., \& Wolfe, V. (1995). Asymmetrical visual-spatial attentional performance in ADHD: Evidence for a right hemispheric deficit. Biological Psychiatry, 37, 789-797.

Casey, B.J., Cohen, J.D., Jezzard, P., Turner, R., Noll, D.C., Trainor, R.J., Giedd, J., Kaysen, D., Hertz-Pannier, L., \& Rapoport, J.L. (1995). Activation of prefrontal cortex in children during a nonspatial working memory task with functional MRI. Neuroimage, 2, 221229. 
Castellanos, F.X., Giedd, J.N., Eckburg, P., Marsh, W.L., Vaituzis, A.C., Kaysen, D., Hamburger, S.D., \& Rapoport, J.L. (1994). Quantitative morphology of the caudate nucleus in attention deficit hyperactivity disorder. American Journal of Psychiatry, 151, 1791-1796.

Chess, S. (1960). Diagnosis and treatment of the hyperactive child. New York State Journal of Medicine, 60, 2379-2385.

Cohen, L.B., \& Younger, B.A. (1984). Infant perception of angular relations. Infant Behavior and Development, 7, 37-47.

Cohen, M.L., Kelly, P.C., \& Atkinson, A.W. (1989). Parent, teacher, child: A trilateral approach to attention deficit disorder. American Journal of the Disabled Child, 143, 1229-1233.

Colby, C.L. (1991). The neuroanatomy and neurophysiology of attention. Journal of Child Neurology, 6 (Supplement), S90-118.

Conners, C.K. (1998). Rating scales in attention-deficit/hyperactivity disorder: Use in assessment and treatment monitoring. Journal of Clinical Psychiatry, 59, 24-30.

Conners, C.K., \& Barkley, R.A. (1985). Rating scales and checklists for child psychopharmacology. Psychopharmacology Bulletin, 21, 809-838.

Corkum, P.V., \& Siegel, L.S. (1993). Is the continuous performance task a valuable research tool for use with children with attention-deficit-hyperactivity disorder? Journal of Child Psychology and Psychiatry, 34, 1217-1239.

Craik, F., \& Lockhart, R. (1972). Levels of processing: A framework for memory research. Journal of Verbal Learning and Verbal Behavior, 11, 671-684.

Denckla, M.B. (1996). Biological correlates of learning and attention: What is relevant to learning disability and attention-deficit hyperactivity disorder? Behavioral Pediatrics, 17, 114-119. 
Dennis, M., Wilkinson, M., Koski, L., \& Humphreys, R.P. (1995). Attention deficits in the long term after childhood head injury. In S.H. Broman \& M.E. Michel (Eds.), Traumatic head injury in children (pp. 165-187). New York: Oxford University Press.

Diamond, A., \& Doar, B. (1989). The performance of human infants on a measure of frontal cortex function: The Delayed Response Task. Developmental Psychobiology, 22, 271294.

Donders, J. (1995, February). Confirmatory factor analysis of the WRAML. Paper presented at the 23rd Annual Meeting of the International Neuropsychological Society, Seattle, WA.

Douglas, V.I. (1972). Stop, look, and listen: The problem of sustained attention and impulse control in hyperactive and normal children. Canadian Journal of Behavioral Science, 4 , $259-282$.

Douglas, V.I. (1983). Attention and cognitive problems. In M. Rutter (Ed.), Developmental neuropsychiatry (pp. 280-329). New York: Guilford Press.

Douglas, V.I., \& Peters, K G. (1979). Toward a clearer definition of the attentional deficit of hyperactive children. In G.A. Hale \& M. Lewis (Eds.), Attention and cognitive development (pp. 173-248). New York: Plenum Press.

Driscoll, M.S. (1992, November). Lateralization of memory functions in children with traumatic brain injury. In W. Adams (Chair), Wide Range Assessment of Memory and Learning: An empirical update. Workshop presented at the 12th Annual Meeting of the National Academy of Neuropsychology, Pittsburgh, PA.

Eliason, M.J., \& Richman, L.C. (1987). The continuous performance test in learning disabled and nondisabled children. Journal of Learning Disabilities, 20, 614-619. 
Epstein, M.A., Shaywitz, S.E., Shaywitz, B.A., \& Woolston, J.L. (1991). The

boundaries of attention deficit disorder. Journal of Learning Disabilities, 24, 78-86.

Faraone, S.V., \& Biederman, J. (1998). Neurobiology of attention-deficit hyperactivity disorder. Biological Psychiatry, 44, 951-958.

Farmer, J.E., Haut, J.S., Williams, J., Kapila, C., Johnstone, B., \& Kirk, K.S. (1999). Comprehensive assessment of memory functioning following traumatic brain injury in children. Developmental Neuropsychology, 15, 269-290.

Fastenau, P.S., Conant, L.L., \& Lauer, R.E. (1998). Working memory in young children: Evidence for modality-specificity and implications for cerebral reorganization in early childhood. Neuropsychologia, 36, 643-652.

Filipek, P.A., Semrud-Clikeman, M., Steingard, R.J., Renshaw, P.F., Kennedy, D.N., \& Biederman, J. (1997). Volumetric MRI analysis comparing subjects having attention-deficit hyperactivity disorder with normal controls. Neurology, 48, 589-601.

Fischer, M. (1996). Erratum regarding medication response of the Gordon Diagnostic System. Journal of Clinical Child Psychology, 25, 121.

Fischer, M., \& Newby, R. (1991). Assessment of stimulant response in ADHD children using a refined multimethod clinical protocol. Journal of Clinical Child Psychology, 20, 232244.

Fletcher, J.M., Ewing-Cobbs, L., Francis, D.J., \& Levin, H.S. (1995). Variability in outcomes after traumatic brain injury in children: A developmental perspective. In S.H. Broman \& M.E. Michel (Eds.), Traumatic head injury in children (pp. 3-21). New York: Oxford University Press. 
Fletcher, J.M., Morris, R.D., \& Francis, D.J. (1991). Methodological issues in the classification of attention-related disorders. Journal of Learning Disabilities, 24, 72-77.

Garcia-Sanchez, C., Estevez-Gonzalez, A., Suarez-Romero, E., \& Junque, C. (1997).

Right hemisphere dysfunction in subjects with attention-deficit disorder with and without hyperactivity. Journal of Child Neurology, 12, 107-115.

Gathercole, S.E. (1998). The development of memory. Journal of Child Psychology and Psychiatry, 39, 3-27.

Gaub, M., \& Carlson, C.L. (1997). Behavioral characteristics of DSM-IV ADHD subtypes in a school-based population. Journal of Abnormal Child Psychology, 25, 103-111.

Giedd, J.N., Castellanos, F.X., Casey, B.J., Kozuch, P., King, A.C., Hamburger, S.D., \& Rapoport, J.L. (1994). Quantitative morphology of the corpus callosum in attention deficit hyperactivity disorder. American Journal of Psychiatry, 151, 665-669.

Ginsburg, H., \& Opper, S. (1969). Piaget's theory of intellectual development: An introduction. Englewood Cliffs, N.J: Prentice-Hall, Inc.

Gioia, G.A. (1998). Re-examining the factor structure of the Wide Range Assessment of Memory and Learning: Implications for clinical interpretation. Assessment, 5, 127-139.

Gleeson, S. (1991). Response acquisition. In I. Iversen \& K.A. Lattal (Eds.), Research methods in the behavioral and neural sciences: Experimental analysis of behavior (pp. 63-86). Amsterdam: Elsevier.

Gordon, M. (1979). The assessment of impulsivity and mediating behaviors in hyperactive and nonhyperactive boys. Journal of Abnormal Child Psychology, 7, 317-326.

Gordon, M. (1983). The Gordon Diagnostic System. DeWitt, NY: Gordon Systems. 
Gordon, M. (1986). How is a computerized attention test used in the diagnosis of attention deficit disorder? Journal of Children in Contemporary Society, 19, 53-64.

Gordon, M., \& Barkley, R.A. (1998). Tests and observational measures. In R A. Barkley, Attention deficit hyperactivity disorder: A handbook for diagnosis and treatment (2nd ed., pp. 294-311). New York: Guilford Press.

Gordon, M., DiNiro, D., \& Mettelman, B.B. (1988). Effect upon outcome of nuances in selection criteria for ADHD/Hyperactivity. Psychological Reports, 62, 539-544.

Gordon, M., DiNiro, D., Mettelman, B.B., \& Tallmadge, J. (1989). Observations of test behavior, quantitative scores, and teacher ratings. Journal of Psychoeducational Assessment, 7. 141-147.

Gordon, M., \& McClure, F.D. (1983, August). The objective assessment of attention deficit disorders. Paper presented at the $91^{\text {st }}$ Annual Convention of the American Psychological Association, Anaheim, CA.

Gordon, M., \& Mettelman, B.B. (1987). Technical guide to the Gordon Diagnostic System. Syracuse, NY: Gordon Systems.

Gordon, M., \& Mettelman, B.B. (1988). The assessment of attention: I. Standardization and reliability of a behavior-based measure. Journal of Clinical Psychology, 44, $682-690$.

Gordon, M., Thomason, D., \& Cooper, S. (1990). To what extent does attention affect K-ABC scores? Psychology in the Schools, 27, 144-147.

Gorenstein, E.E., Mammato, C.A., \& Sandy, J.M. (1989). Performance of inattentiveoveractive children on selected measures of prefrontal-type function. $\underline{\text { Journal of Clinical }}$ Psychology, 45, 619-632. 
Grant, M.L., Ilai, D., Nussbaum, N.L., \& Bigler, E.D. (1990). The relationship between continuous performance tasks and neuropsychological tests in children with attention-deficit hyperactivity disorder. Perceptual and Motor Skills, 70, 435-445.

Grodzinsky, G.M., \& Diamond, R. (1992). Frontal lobe functioning in boys with attention deficit hyperactivity disorder. Developmental Neuropsychology, 8, 427-445.

Hair, J.F., Anderson, R.E., Tatham, R.L., \& Black, W.C. (1998). Multivariate data analysis (Fifth edition). Upper Saddle River, NJ: Prentice-Hall, Inc.

Hale, S., Bronik, M.D., \& Fry, A.F. (1997). Verbal and spatial working memory in school-age children: Developmental differences in susceptibility to interference. Developmental Psychology, 33, 364-371.

Halford, G.S., Maybery, M.T., O’Hare, A.W., \& Grant, P. (1994). The development of memory and processing capacity. Child Development, 65, 1338-1356.

Hall, C.W., \& Kataria, S. (1992). Effects of two treatment techniques on delay and vigilance tasks with attention deficit hyperactive disorder (ADHD) children. Journal of Psychology, 126, 17-25.

Halperin, J.M., Matier, K., Bedi, G., Sharma, V., \& Newcorn, J.H. (1992). Specificity of inattention, impulsivity, and hyperactivity to the diagnosis of attention-deficit hyperactivity disorder. Journal of the American Academy of Child and Adolescent Psychiatry, 31, 190-196.

Halperin, J.M., Newcorn, J.H., Sharma, V., Healey, J.M., Wolf, L.E., Pascualvaca, D.M., \& Schwartz, S. (1990). Inattentive and noninattentive ADHD children: Do they constitute a unitary group? Journal of Abnormal Child Psychology, 18, 437-449.

Halperin, J.M., Wolfe, L.E., Pascualvaca, D.M., Newcorn, J.H., Healey, J.M., O'Brien, J.D., Morganstein, A., \& Young, J.G. (1988). Differential assessment of attention and 
impulsivity in children. Journal of the American Academy of Child and Adolescent Psychiatry, 27, 326-329.

Harnadek, M.C., \& Rourke, B.P. (1994). Principal identifying features of the syndrome of nonverbal learning disabilities in children. Journal of Learning Disabilities, 27, 144-154.

Harper, G.W., \& Ottinger, D.R. (1992). The performance of hyperactive and control preschoolers on a new computerized measure of visual vigilance: The Preschool Vigilance Task. Journal of Child Psychology and Psychiatry, 33, 1365-1372.

Hartshorn, K., Rovee-Collier, C., Gerhardstein, P., Bhatt, R.S., Klein, P.J., Aaron, F., Wondoloski, T.L., \& Wurtzel, N. (1998). Developmental changes in the specificity of memory over the first year of life. Developmental Psychobiology, 33, 61-78.

Hashimoto, N. (1991). Memory development in early childhood: Encoding process in a spatial task. Journal of Genetic Psychology, 152, 101-117.

Haut, J.S., Haut, M.W., Callahan, T.S., \& Franzen, M.D. (1992, November). Factor analysis of the Wide Range Assessment of Memory and Learning (WRAML) scores in a clinical sample. Paper presented at the 12th Annual Meeting of the National Academy of Neuropsychology, Pittsburgh, PA.

Haut, J.S., Haut, M.W., \& Franzen, M.D. (1992, February). Assessment of an attentional component of Wide Range Assessment of Memory and Learning (WRAML) subtests. Paper presented at the 20th Annual Meeting of the International Neuropsychological Society, San Diego, CA.

Haut, J.S., Warren, D.A., \& Bretzman, Y. (1994, February). Performance differences of older children on the WRAML Story Memory subtest: Are stories B and C different? Paper 
presented at the 22nd Annual Meeting of the International Neuropsychological Society,

Cincinnati, $\mathrm{OH}$.

Haut, J., Williams, J., \& Hendon, A. (1994, February). Memory and attention skills of children with neurological and psychiatric disorders: Implications for clinical utility of the

WRAML. Paper presented at the 22nd Annual Meeting of the International Neuropsychological Society, Cincinnati, OH.

Herschkowitz, N., Kagan, J., \& Zilles, K. (1997). Neurobiological bases of behavioral development in the first year. Neuropediatrics, 28, 296-306.

Hill, D.E., Ciesielski, K.T., Sethre-Hofstad, L., Duncan, M.H., \& Lorenzi, M. (1997). Visual and verbal short-term memory deficits in childhood leukemia survivors after intrathecal chemotherapy. Journal of Pediatric Psychology, 22, 861-870.

Hooper, S.R., Linz, T.D., Tramontana, M.G., \& Stein, M. (1992, November). Dimensions of inattention and impulsivity and their relationship to memory functions in children and adolescents. Paper presented at the 12th Annual Meeting of the National Academy of Neuropsychology, Pittsburgh, PA.

Hothersall, D. (1990). History of psychology (2nd ed.). New York: McGraw-Hill. Howe, M.L., \& Courage, M.L. (1997). Independent paths in the development of infant learning and forgetting. Journal of Experimental Child Psychology, 67, 131-163.

Hynd, G.W., Hern, K.L., Voeller, K.K., \& Marshall, R.M. (1991). Neurobiological basis of attention-deficit hyperactivity disorder (ADHD). School Psychology Review, 20, 174-186.

Inoue, K., Nadaoaka, T., Oiji, A., Morioka, Y., Totsuka, S., Kanbayashi, Y., \& Hukui, T. (1998). Clinical evaluation of attention-deficit hyperactivity disorder by objective quantitative measures. Child Psychiatry and Human Development, 28, 179-188. 
Jastak, S., \& Wilkinson, G.S. (1984). Wide Range Achievement Test-Revised:

Administration Manual. Wilmington, DE: Jastak Associates.

Jenkins, J.J. (1979). Four points to remember: A tetrahedral model of memory experiments. In L.S. Cermak \& F.I.M. Craik (Eds.), Levels of processing in human memory (pp. 429-446). Hillsdale, NJ: Erlbaum.

Kagan, J. (1979). The form of early development: Continuity and discontinuity in emergent competencies. Archives of General Psychiatry, 36, 1047-1054.

Kail, R. (1990). The development of memory in children (3rd ed.). New York: Freeman.

Kaplan, B.J., Dewey, D., Crawford, S.G., \& Fisher, G.C. (1998). Deficits in long-term memory are not characteristic of ADHD. Journal of Clinical and Experimental Neuropsychology, 20, 518-528.

Karatekin, C., \& Asarnow, R.F. (1998). Working memory in childhood-onset schizophrenia and attention-deficit/hyperactivity disorder. Psychiatry Research, 80, 165-176.

Kashden, J., Haut, J.S., Wong, S., \& Franzen, M.D. (1994, February). Commission errors on the WRAML Picture Memory subtest: Relation to performance. Paper presented at the $22^{\text {nd }}$ Annual Meeting of the International Neuropsychological Society, Cincinnati, OH.

Kataria, S., Hall, C.W., Wong, M.M., \& Keys, G.F. (1992). Learning styles of LD and NLD ADHD children. Journal of Clinical Psychology, 48, 371-378.

Kaufman, A.S. (1979). Intelligent testing with the WISC-R. New York: Wiley. Kaufmann, P.M., \& Espy, K.A. (1992, August). Comparison of the WRAML General Memory and Screening Indices. Paper presented at the 100th Annual Convention of the American Psychological Association, Washington, DC. 
Kaufmann, P.M., Fletcher, J.M., Levin, H.S., Miner, M.E., \& Ewing-Cobbs, L. (1993). Attentional disturbance after pediatric closed head injury. Journal of Child Neurology, 8, 348353.

Kennedy, M.L., \& Guilmette, T.J. (1995, August). The relationship between the WRAML Memory Screening and General Memory Indices in a clinical population. Paper presented at the 103rd Annual Convention of the American Psychological Association, New York, NY.

Kertesz, A., Nicholson, I., Cancelliere, A., Kassa, K., \& Black, S.E. (1985). Motor impersistence: A right hemisphere syndrome. Neurology, 35, 662-666.

Klee, S.H., \& Garfinkel, B.D. (1983). The computerized continuous performance task: A new measure of inattention. Journal of Abnormal Child Psychology, 11, 487-496.

Korkman, M., \& Peltomaa, K. (1991). A pattern of test findings predicting attention problems at school. Journal of Abnormal Child Psychology, 19, 451-467.

Korkman, M. \& Pesonen, A.E. (1994). A comparison of neuropsychological test profiles of children with attention-deficit-hyperactivity disorder and/or learning disorder. Journal of Learning Disabilities, 27, 383-392.

Lahey, B.B., Applegate, B., McBurnett, K., Biederman, J., Greenhill, L., Hynd, G., Barkley, R.A., Newcorn, J., Jensen, P., Richters, J., Garfinkel, B., Kerdyk, L., Frick, P.J., Ollendick, T., Perez, D., Hart, E., Waldman, I., \& Shaffer, D. (1994). DSM-IV field trials for attention deficit/hyperactivity disorder in children and adolescents. American Journal of Psychiatry, 152, 1673-1685.

Lambert, N.M., \& Sandoval, J. (1980). The prevalence of learning disabilities in a sample of children considered hyperactive. Journal of Abnormal Child Psychology, 8, 33-50. 
Attention and Memory in Children

Lazar, J.W., \& Frank, Y. (1998). Frontal systems dysfunction in children with attentiondeficit/hyperactivity disorder and learning disabilities. Journal of Neuropsychiatry and Clinical Neuroscience, 10, 160-167.

Levy, F., \& Hobbes, G. (1989). Reading, spelling, and vigilance in attention deficit and conduct disorder. Journal of Abnormal Child Psychology, 17, 291-298.

Levy, F. \& Hobbes, G. (1997). Discrimination of attention deficit hyperactivity disorder by the continuous performance test. Journal of Paediatrics and Child Health, 33, 384-387.

Loge, D.V., Staton, R.D., \& Beatty, W.W. (1990). Performance of children with ADHD on tests sensitive to frontal lobe dysfunction. Journal of the American Academy of Child and Adolescent Psychiatry, 29, 540-545.

Lorsbach, T.C., \& Reimer, J.F. (1997). Developmental changes in the inhibition of previously relevant information. Journal of Experimental Child Psychology, 64, 317-342.

Losier, B.J., McGrath, P.J., \& Klein, R.M. (1996). Error patterns on the continuous performance test in non-medicated and medicated samples of children with and without ADHD: A meta-analytic review. Journal of Child Psychology and Psychiatry, 37, 971-987.

Luciana, M., \& Nelson, C.A. (1998). The functional emergence of prefrontally-guided working memory systems in four- to eight-year-old children. Neuropsychologia, 36, 273-293.

Mataro, M., Garcia-Sanchez, C., Junque, C., Estevez-Gonzalez, A., \& Pujol, J. (1997). Magnetic resonance imaging measurement of the caudate nucleus in adolescents with attentiondeficit hyperactivity disorder and its relationship with neuropsychological and behavioral measures. Archives of Neurology, 54, 963-968.

Max, J.E., Arndt, S., Castillo, C.S., Bokura, H., Robin, D.A., Lindgren, S.D., Smith, W.L., Sato, Y., \& Mattheis, P.J. (1998). Attention-deficit hyperactivity symptomatology after 
traumatic brain injury: A prospective study. Journal of the American Academy of Child and Adolescent Psychiatry, 37, 841-847.

McCarthy, D.A. (1972). Manual for the McCarthy Scales of Children's Abilities. San Antonio, TX: The Psychological Corporation.

McClure, F.D., \& Gordon, M. (1984). Performance of disturbed hyperactive and nonhyperactive children on an objective measure of hyperactivity. Journal of Abnormal Child Psychology, 12, 561-571.

McGee, R., \& Share, D.L. (1988). Attention deficit disorder-hyperactivity and academic failure: Which comes first and what should be treated? Journal of the American Academy of Child and Adolescent Psychiatry, 27, 318-325.

McGee, R., Williams, S., Moffitt, T., \& Anderson, J. (1989). A comparison of 13-yearold boys with attention deficit and/or reading disorder on neuropsychological measures. $\underline{\text { Journal }}$ of Abnormal Child Psychology, 17, 37-53.

McKittrick, T., Haut, J.S., Schauss, S., Bradlyn, A.S., \& Franzen, M.D. (1994, February). Predicting WRAML performance based on intellectual functioning: Evidence for construct validity of the WRAML. Paper presented at the 22nd Annual Meeting of the International Neuropsychological Society, Cincinnati, $\mathrm{OH}$.

McMillan, D.N., Walters, E., \& Holder, A.H. (1993, August). Developing a battery for assessing attention deficits in children. Paper presented at the $101^{\text {st }}$ Annual Convention of the American Psychological Association, Toronto, Canada.

Myers, N.A., Perris, E.E., \& Speaker, C.J. (1994). Fifty months of memory: A longitudinal study in early childhood. Memory, 2, 383-415. 
Newcorn, J.H., Halperin, J.M., Schwartz, S., Pascualvaca, D., Schmeidler, J., \& Sharma, V. (1994). Parent and teacher ratings of attention-deficit hyperactivity disorder symptoms: Implications for case identification. Journal of Developmental and Behavioral Pediatrics, 15, 8691.

Niedermeyer, E. (1998). Frontal lobe functions and dysfunctions. Clinical Electroencephalography, 29, 79-90.

Nigg, J.T., Hinshaw, S.P., Carte, E.T., \& Treuting, J.J. (1998). Neuropsychological correlates of childhood attention-deficit/hyperactivity disorder: Explainable by comorbid disruptive behavior or reading problems? Journal of Abnormal Psychology, 107, 468-480.

Oades, R.D. (1998). Frontal, temporal and lateralized brain function in children with attention-deficit hyperactivity disorder: A psychophysiological and neuropsychological viewpoint on development. Behavior and Brain Research, 94, 83-95.

O'Dougherty, M., Nuechterlein, K.H., \& Drew, B. (1984). Hyperactive and hypoxic children: Signal detection, sustained attention, and behavior. Journal of Abnormal Psychology, 93, 178-191.

Olds, J., \& Schlieper, A. (1994, November). Clinical utility of the Wide Range Assessment of Memory and Learning. Paper presented at the 14th Annual Meeting of National Academy of Neuropsychology, Orlando, FL.

O'Neill, M.E., \& Douglas, V.I. (1991). Study strategies and story recall in attention deficit disorder and reading disability. Journal of Abnormal Child Psychology, 19, 671-692.

O'Neill, M.E., \& Douglas, V.I. (1996). Rehearsal strategies and recall performance in boys with and without attention deficit hyperactivity disorder. Journal of Pediatric Psychology, $\underline{21,} 73-88$. 
Ong, L.C., Chandran, V., Zasmani, S., \& Lye, M.S. (1998). Outcome of closed head injury in Malaysian children: Neurocognitive and behavioural sequelae. Journal of Paediatrics and Child Health, 34, 363-368.

Parasuraman, R., \& Davies, D.R. (1984). Varieties of attention. Toronto: Academic Press.

Pardo, J.V., Fox, P.T., \& Raichle, M.E. (1991). Localization of a human system for sustained attention by positron emission tomography. Nature, 349, 61-64.

Parker, R.S. (1994). Neurobehavioral outcome of children's mild traumatic brain injury. Seminars in Neurology, 14, 67-73.

Pascual-Leone, J. (1970). A mathematical model for the transition rule in Piaget's developmental stages. Acta Psychologica, 32, 301-345.

Pastore, R.E., \& Scheirer, C.J. (1974). Signal detection theory: Considerations for general application. Psychological Bulletin, 81, 945-958.

Phelps, L. (1996). Discriminative validity of the WRAML with ADHD and LD children. Psychology in the Schools, 33, 5-12.

Posner, M.I., \& Petersen, S.E. (1990). The attention system of the human brain. Annual Reviews in Neuroscience, 13, 25-42.

Posner, M.I., \& Rafal, R.D. (1987). Cognitive theories of attention and the rehabilitation of attentional deficits. In M.J. Meier, A.L. Benton, \& L. Diller (Eds.), Neuropsychological rehabilitation (pp. 182-201). New York: Guilford Press.

Post, E.M., Burko, M.S., \& Gordon, M. (1990). Single-component microcomputerdriven assessment of attention. Behavior Research Methods, Instruments, \& Computers, 22, 297-301. 
Power, T.J. (1992). Contextual factors in vigilance testing of children with ADHD. Journal of Abnormal Child Psychology, 20, 579-593.

Quay, H.C. (1988). Attention deficit disorder and the behavioral inhibition system: The relevance of the neuropsychological theory of Jeffrey A. Gray. In L. Bloomingdale \& J. Sergeant (Eds.), Attention deficit disorder: Criteria, cognition, and intervention (pp. 117-126). New York: Pergamon Press.

Quay, H.C. (1997). Inhibition and attention deficit hyperactivity disorder. Journal of Abnormal Child Psychology, 25, 7-14.

Rapport, M.D. (1992). Treating children with attention-deficit hyperactivity disorder. Behavior Modification, 16, 155-163.

Rapport, M.D., DuPaul, G.J., Stoner, G., \& Jones, J.T. (1986). Comparing classroom and clinic measures of attention deficit disorder: Differential, idiosyncratic, and dose-response effects of methylphenidate. Journal of Consulting and Clinical Psychology, 54, 334-341.

Rapport, M.D., Jones, J.T., DuPaul, G.J., Kelly, K.L., Gardner, M.J., Tucker, S.B., \& Shea, M.S. (1987). Attention deficit disorder and methylphenidate: Group and single-subject analyses of dose effects on attention in clinic and classroom settings. Journal of Clinical Child Psychology, 16, 329-338.

Reid, R., \& Maag, J.W. (1994). How many fidgets in a pretty much: A critique of behavior rating scales for identifying students with ADHD. Journal of School Psychology, 32, $339-354$.

Richards, G.P., Samuels, S.J., Turnure, J.E., \& Ysseldyke, J.E. (1990). Sustained and selective attention in children with learning disabilities. Journal of Learning Disabilities, 23, 129-136. 
Robins, P.M. (1992). A comparison of behavioral and attentional functioning in children diagnosed as hyperactive or learning-disabled. Journal of Abnormal Child Psychology, 20, 6582.

Rogeness, G.A., Javors, M.A., \& Pliszka, S.R. (1992). Neurochemistry and child and adolescent psychiatry. Journal of the American Academy of Child and Adolescent Psychiatry, 31, 765-781.

Rogers, A.K., \& Gridley, B.E. (1995, February). The internal structure of the Wide Range Assessment of Learning and Memory: Who's right? Paper presented at the 23rd Annual Meeting of the International Neuropsychological Society, Seattle, WA.

Rose, S.A., \& Feldman, J.F. (1997). Memory and speed: Their role in the relation of infant information processing to later IQ. Child Development, 68, 630-641.

Rosvold, H.E., Mirsky, A.F., Sarason, I., Bransome, E.D., \& Beck, L.H. (1956). A continuous performance test of brain damage. Journal of Consulting Psychology, 20, 343-350. Rourke, B.P. (1989). Nonverbal learning disabilities: The syndrome and model. New York: Guilford Press.

Ryan, C.M. (1990). Age-related improvement in short-term memory efficiency during adolescence. Developmental Neuropsychology, 6, 193-205.

Safer, D.J., \& Allen, R.P. (1976). Hyperactive children: Diagnosis and management. Baltimore, MD: University Park Press.

Sattler, J.M. (1992). Assessment of children (Revised and updated 3rd Ed.). San Diego, CA: Author.

Satz, P. (1993). Brain reserve capacity on symptom onset after brain injury: A formulation and review of evidence for threshold theory. Neuropsychology, 7, 273-295. 
Schachar, R.J., Tannock, R., \& Logan, G. (1993). Inhibitory control, impulsiveness, and attention deficit hyperactivity disorder. Clinical Psychology Review, 13, 721-739.

Schaughency, E.A., \& Rothlind, J. (1991). Assessment and classification of attention deficit hyperactive disorders. School Psychology Review, 20, 187-202.

Schneider, W., \& Pressley, M. (1989). Memory development between 2 and 20. New York: Springer-Verlag.

Scoville, W.B., \& Milner, B. (1957). Loss of recent memory after bilateral hippocampal lesions. Journal of Neurology, Neurosurgery, and Psychiatry, 20, 11-21.

Seidman, L.J., Biederman, J., Faraone, S.V., Weber, W., \& Ouellette, C. (1997). Toward defining a neuropsychology of attention deficit-hyperactivity disorder: Performance of children and adolescents from a large clinically referred sample. Journal of Consulting and Clinical Psychology, 65, 150-160.

Semrud-Clikeman, M., Biederman, J., Sprich-Buckminster, S., Krifcher-Lehman, B., Faraone, S.V., \& Norman, D. (1992). Comorbidity between ADDH and learning disability: A review and report in a clinically referred sample. Journal of the American Academy of Child and Adolescent Psychiatry, 31, 439-448.

Sergeant, J.A. (1995). A theory of attention: An information processing perspective. In G.R. Lyon \& N.A. Krasnegor (Eds.), Attention, memory, and executive function (pp. 57-69). Baltimore, MD: Paul H. Brookes.

Sergeant, J.A., \& van der Meere, J. (1988). What happens when the hyperactive child commits an error? Psychiatry Research, 24, 157-164.

Shallice, T. (1982). Specific impairments of planning. Philosophical Transcripts of the Royal Society of London Basic Biological Sciences, 298, 199-209. 
Sheslow, D., \& Adams, W. (1990). Wide Range Assessment of Memory and Learning:

Administration Manual. Wilmington, DE: Jastak Associates.

Shue, K.L., \& Douglas, V.I. (1992). Attention deficit hyperactivity disorder and the frontal lobe syndrome. Brain and Cognition, 20, 104-124.

Silberstein, R.B., Farrow, M., Levy, F., Pipingas, A., Hay, D.A., \& Jarman, F.C. (1998). Functional brain electrical activity mapping in boys with attention-deficit/hyperactivity disorder. Archives of General Psychiatry, 55, 1105-1112.

Skinner, B.F. (1953). Science and human behavior. New York: Free Press.

Sonuga-Barke, E.J.S., Taylor, E., \& Heptinstall, E. (1992). Hyperactivity and delay aversion - II. The effect of self versus externally imposed stimulus presentation periods on memory. Journal of Clinical Psychology and Psychiatry, 33, 399-409.

SPSS, Inc. (1997). SPSS Version 7.5.2. Chicago, IL: Author.

Squire, L. (1987). Memory and brain. New York: Oxford University Press.

Sroufe, L.A., Cooper, R.G., \& DeHart, G.B. (1996). Child development: Its nature and course (3rd ed.). New York: McGraw-Hill.

Stone, M. (1990, August). Psychometric properties of the Wide Range Assessment of Memory and Learning (WRAML). Paper presented at the 98th Annual Convention of the American Psychological Association, Boston, MA.

Stone, M.H., \& Wright, B.D. (1981). Knox's Cube Test, Junior and Senior Version: Instruction Manual. Chicago, IL: Stoelting.

Strandburg, R.J., Marsh, J.T., Brown, W.S., Asarnow, R.F., Higa, J., Harper, R., \& Guthrie, D. (1996). Continuous-processing: Event-related potentials in children with attention deficit hyperactivity disorder. Biological Psychiatry, 40, 964-980. 
Swanson, H.L. (1996). Individual and age-related differences in children's working memory. Memory and Cognition, 24, 70-82.

Swanson, J., Castellanos, F.X., Murias, M., LaHoste, G., \& Kennedy, J. (1998).

Cognitive neuroscience of attention deficit hyperactivity disorder and hyperkinetic disorder. Current Opinion in Neurobiology, 8, 263-271.

Swanson, L. (1983). A developmental study of vigilance in learning disabled and nondisabled children. Journal of Abnormal Child Psychology, 11, 415-429.

Sykes, D.H., Douglas, V.I., \& Morganstern, G. (1973). Sustained attention in hyperactive children. Journal of Child Psychology and Psychiatry, 14, 213-220.

Tabachnick, B.G., \& Fidell, L.S. (1996). Using multivariate statistics (3rd edition). New York: Harper Collins.

Taylor, H.G., \& Schatschneider, C. (1992). Child neuropsychological assessment: A test of basic assumptions. The Clinical Neuropsychologist, 6, 259-275.

Teasdale, G., \& Jennett, B. (1974). Assessment of coma and impaired consciousness: A practical scale. Lancet, 2, 81-84.

Thorndike, R.L., Hagen, E.P., \& Sattler, J.M. (1986). Guide for administering and scoring the Stanford-Binet Intelligence Scale: Fourth Edition. Chicago, IL: Riverside Publishing.

Tirosh, E., \& Cohen, A. (1998). Language deficit with attention-deficit disorder: A prevalent comorbidity. Journal of Child Neurology, 13, 493-497.

Trommer, B.L., Hoeppner, J.B., Lorber, R., \& Armstrong, K.J. (1988a). The Go -- NoGo paradigm in attention deficit disorder. Annals of Neurology, 24, 610-614. 
Trommer, B.L., Hoeppner, J.B., Lorber, R., \& Armstrong, K. (1988b). Pitfalls in the use of a continuous performance test as a diagnostic tool in attention deficit disorder.

Developmental and Behavioral Pediatrics, 9, 339-345.

Trommer, B.L., Lorber, R., Armstrong, K., \& Hoeppner, J.B. (1987). Neuropsychologic correlates of the Vigilance Task. The ADD/Hyperactivity Newsletter, 7, 3-5.

Tryon, W.W. (1993). The role of motor excess and instrumented activity measurement in attention deficit hyperactivity disorder. Behavior Modification, 17, 371-406.

van der Meere, J., \& Sergeant, J. (1988). Controlled processing and vigilance in hyperactivity: Time will tell. Journal of Abnormal Child Psychology, 16, 641-655.

van der Meere, J., Wekking, E., \& Sergeant, J. (1991). Sustained attention and pervasive hyperactivity. Journal of Child Psychology, Psychiatry, and Allied Disciplines, 32, 275-284.

van Zomeren, A.H., \& Brouwer, W.H. (1994). Clinical neuropsychology of attention. New York: Oxford University Press.

Warner, M.H. (1992, December). Memory in children and adolescents with seizures: Usefulness of the Wide Range Assessment of Memory and Learning (WRAML). Paper presented at the Annual Meeting of the American Epilepsy Society, Seattle, WA.

Waschbusch, D.A., Kipp, H.L., \& Pelham, W.E. (1998). Generalization of behavioral and psychostimulant treatment of attention-deficit/hyperactivity disorder (ADHD): Discussion and examples. Behaviour Research and Therapy, 36, 675-694.

Wasserman, J.D., \& Cambias, R.D. (1993, October). WRAML factor-derived deviation quotients: Tables for clinical use. Paper presented at the 13th Annual Meeting of the National Academy of Neuropsychology, Phoenix, AZ. 
Wechsler, D. (1974). Manual for the Wechsler Intelligence Scale for Children-Revised.

San Antonio, TX: The Psychological Corporation.

Wechsler, D. (1981). Manual for the Wechsler Adult Intelligence Scale-Revised. San Antonio, TX: The Psychological Corporation.

Wechsler, D. (1987). Manual for the Wechsler Memory Scale-Revised. San Antonio, TX: The Psychological Corporation.

Wechsler, D. (1991). Manual for the Wechsler Intelligence Scale for Children-Third Edition. San Antonio, TX: The Psychological Corporation.

Welsh, M.C., \& Pennington, B.F. (1988). Assessing frontal lobe functioning in children: Views from developmental psychology. Developmental Neuropsychology, 4, 199-230.

Wherry, J.N., Paal, N., Jolly, J.B., Adam, B., Holloway, C., Everett, B., \& Vaught, L. (1993). Concurrent and discriminant validity of the Gordon Diagnostic System: A preliminary study. Psychology in the Schools, 30, 29-36.

Whyte, J. (1992). Attention and arousal: Basic science aspects. Archives of Physical Medicine and Rehabilitation, 73, 940-949.

Whyte, J., Polansky, M., Cavallucci, C., Fleming, M., Lhulier, J., \& Coslett, H.B. (1996). Inattentive behavior after traumatic brain injury. Journal of the International Neuropsychological Society, 2, 274-281.

Williams, D.H., Levin, H.S., \& Eisenberg, H.M. (1990). Mild head injury classification. Neurosurgery, 27, 422-428.

Williams, J., Griebel, M.L., \& Dykman, R.A. (1998). Neuropsychological patterns in pediatric epilepsy. Seizure, 7, 223-228. 
Williams, J., \& Haut, J.S. (1995). Differential performances on the WRAML in children and adolescents diagnosed with epilepsy, head injury, and substance abuse. Developmental Neuropsychology, 11, 201-213.

Williams, J., Sharp, G., \& Griebel, M. (1992, December). Memory performance in children with epilepsy on the WRAML. Paper presented at the Annual Meeting of the American Epilepsy Society, Seattle, WA.

Wolfe, J.N. (1996). Relations of cognitive functions associated with the frontal lobes and learning disorders in children. Psychological Reports, 79, 323-333.

Wolraich, M.L., Hannah, J.N., Pinnock, T.Y., Baumgaertel, A., \& Brown, J. (1996). Comparison of diagnostic criteria for attention deficit hyperactivity disorder in a country-wide sample. Journal of the American Academy of Child and Adolescent Psychiatry, 35, 319-324. Yeates, K.O., Blumenstein, E., Patterson, C.M.., \& Delis, D. (1995). Verbal learning and memory following pediatric closed-head injury. Journal of the International Neuropsychological Society, 1, 78-87.

Zametkin, A.J., \& Liotta, W. (1998). The neurobiology of attention-deficit/hyperactivity disorder. Journal of Clinical Psychiatry, 59 (Supplement 7), 17-23. 
Attention and Memory in Children

\section{Appendix A}

Data Questionnaire for Control Subjects 


\section{HISTORY QUESTIONS:}

(If the response is "yes", please include a brief explanatory comment.)

1. Date of child's birth:

2 Child's grade in school:

3. Educational History:

Has your child repeated a grade?

Has your child had any special education placement?

Have you been told that your child has a learning disability?

4. Medical history:

Does your child have any chronic illnesses?

Has your child had a head injury?

Does your child have a seizure disorder?

Has your child had any other neurological illness?

Is your child taking any medications?

Has your child been treated for attention deficit/ hyperactivity disorder?

Has your child been treated for other behavioral disorders?

Has your child been treated for thought disorder or other psychiatric illness?

Does your child often drink alcohol or use drugs?

Has your child been exposed to a poison (e.g., lead, gasoline fumes)?

Are there any concerns about your child's birth?

Are there any other medical concerns regarding your child? 
5. Family history:

Highest education level attained for --Mother:

Father:

Parental occupation ---

Mother:

Father:

Marital status of parents:

6. Gender of child:

7. Race of child: 
Table 1

Subject Groups and Characteristics

\begin{tabular}{|c|c|c|c|c|}
\hline $\begin{array}{r}\text { Groups: } \\
\end{array}$ & \multirow[t]{2}{*}{ ADHD $^{\mathrm{a}}$} & \multirow[t]{2}{*}{ LD/ADHD ${ }^{b}$} & \multirow[t]{2}{*}{$\mathbf{T B I}^{\mathrm{c}}$} & \multirow[t]{2}{*}{ Control } \\
\hline Characteristics: & & & & \\
\hline Gender & $\begin{array}{l}23 \text { males } \\
2 \text { females }\end{array}$ & $\begin{array}{l}21 \text { males } \\
4 \text { females }\end{array}$ & $\begin{array}{l}21 \text { males } \\
4 \text { females }\end{array}$ & $\begin{array}{l}8 \text { males } \\
17 \text { females }\end{array}$ \\
\hline Race & $\begin{array}{c}\text { 21 Caucasian } \\
\text { 4 African-American }\end{array}$ & $\begin{array}{c}23 \text { Caucasian } \\
2 \text { African-American }\end{array}$ & 25 Caucasian & $\begin{array}{c}23 \text { Caucasian } \\
2 \text { African-American }\end{array}$ \\
\hline Age range $^{\mathrm{d}}$ & $\begin{array}{c}9-0-27 \text { to } 14-11-15 \\
(X=11-9)\end{array}$ & $\begin{array}{l}9-2-4 \text { to } 14-11-20 \\
(X=11-10)\end{array}$ & $\begin{array}{c}9-0-1 \text { to } 14-7-2 \\
(X=12-8)\end{array}$ & $\begin{array}{c}9-5-24 \text { to } 14-11-23 \\
(X=12-1)\end{array}$ \\
\hline $\begin{array}{l}\text { Mean FSIQ scores } \\
\text { (Standard Deviations) }\end{array}$ & $\begin{array}{l}95.04 \\
(7.28)\end{array}$ & $\begin{array}{l}94.16 \\
(9.41)\end{array}$ & $\begin{array}{c}90.32 \\
(11.66)\end{array}$ & $\begin{array}{l}102.84 \\
(7.70)\end{array}$ \\
\hline Educational support $^{\mathrm{e}}$ & 12 subjects & 22 subjects & $\begin{array}{l}7 \text { subjects, pre-injury } \\
7 \text { subjects, post-injury }\end{array}$ & 0 subjects \\
\hline Grade retention & 5 subjects & 6 subjects & $\begin{array}{l}8 \text { subjects, pre-injury } \\
0 \text { subjects, post-injury }\end{array}$ & 0 subjects \\
\hline ADHD subtype ${ }^{f}$ & $\begin{array}{l}6 \text { Combined } \\
18 \text { Inattentive } \\
1 \text { Imp/Hyper }\end{array}$ & $\begin{array}{l}5 \text { Combined } \\
18 \text { Inattentive } \\
2 \text { Imp/Hyper }\end{array}$ & N/A & N/A \\
\hline $\begin{array}{l}\text { Suspected comorbid } \\
\text { diagnoses }^{\text {g }}\end{array}$ & 5 subjects & 11 subjects & 9 subjects, post-injury & 0 subjects \\
\hline Medications $^{\mathrm{h}}$ & $\begin{array}{c}9 \text { subjects }^{\mathrm{I}} \\
5=\text { day of eval }\end{array}$ & $\begin{array}{c}5 \text { subjects }^{\mathrm{j}} \\
2=\text { day of eval }\end{array}$ & $\begin{array}{c}2 \text { subjects }^{\mathrm{k}} \\
2=\text { day of eval }\end{array}$ & 0 subjects \\
\hline
\end{tabular}

${ }^{a} \mathrm{ADHD}=$ Attention Deficit Hyperactivity Disorder. ${ }^{\mathrm{b}} \mathrm{LD} / \mathrm{ADHD}=$ comorbid Learning Disorder/Attention Deficit Hyperactivity Disorder. ${ }^{\mathrm{c}} \mathrm{TBI}=$ Traumatic Brain Injury. ${ }^{\mathrm{d}}$ Age ranges in years-months-days. ${ }^{\mathrm{e}} \mathrm{Special}$ education or compensatory education. ${ }^{\mathrm{f}}$ Combined $=$ Inattentive plus Impulsive/Hyperactive type; Imp/Hyper = Impulsive/Hyperactive type. ${ }^{9}$ No confirmed diagnoses for any subject: suspected Oppositional Defiant Disorder, psychosocial stressors, Depression. ${ }^{\mathrm{h}}$ Currently prescribed. ${ }^{\mathrm{i}}$ All stimulants. ${ }^{\mathrm{j}} 4=$ stimulants only, $1=$ plus Tegretol, $2=$ plus melatonin or antihistamines for sleep. ${ }^{\mathrm{k}} 1=\mathrm{Ritalin}, 1=\mathrm{BuSpar}$. 
Table 2

TBI Group: Subject Characteristics

\begin{tabular}{|c|c|c|}
\hline Type of accident: & $\begin{array}{l}\text { Motor vehicle (MVA) } \\
\text { MVA/Pedestrian or bike } \\
\text { Bike/3-4wheeler } \\
\text { Fall } \\
\text { Assault }\end{array}$ & $\begin{array}{l}5 \text { subjects } \\
8 \text { subjects } \\
7 \text { subjects } \\
4 \text { subjects } \\
1 \text { subjects }\end{array}$ \\
\hline Time unconscious: & $\begin{array}{l}0 \text { minutes } \\
\text { to } 15 \text { minutes } \\
\text { to } 30 \text { minutes } \\
1-2 \text { hours } \\
2-24 \text { hours } \\
\text { over } 24 \text { hours } \\
\text { unknown }\end{array}$ & $\begin{array}{l}8 \text { subjects } \\
1 \text { subject } \\
2 \text { subjects } \\
1 \text { subject } \\
1 \text { subject } \\
3 \text { subjects (range=28 hrs }-14 \text { days) } \\
9 \text { subjects }\end{array}$ \\
\hline $\begin{array}{l}\text { Glasgow Coma Scale } \\
\text { score: }\end{array}$ & $\begin{array}{l}\text { Mild (13-15) } \\
\text { Moderate (9-12) } \\
\text { Severe (3-8) } \\
\text { Unknown }\end{array}$ & $\begin{array}{l}4 \text { subjects } \\
3 \text { subjects } \\
1 \text { subject } \\
17 \text { subjects }\end{array}$ \\
\hline Structural findings: & $\begin{array}{c}\text { Positive CT/MRI } \\
\text { findings for } 19 \text { subjects }\end{array}$ & \\
\hline Time since accident: & $\begin{array}{c}\text { Range }=9 \text { days to } 9 \text { years } \\
(X=26 \text { months, } \\
\text { median }=12 \text { months })\end{array}$ & \\
\hline
\end{tabular}


Table 3

LD/ADHD Group: LD Characteristics

\begin{tabular}{|l|c|}
\hline \multicolumn{1}{|c|}{ Type of Learning Disability } & Number of Subjects \\
\hline Reading \& Spelling & 6 \\
\hline Math & 2 \\
\hline $\begin{array}{c}\text { Written Language } \\
\text { (written expression) }\end{array}$ & 2 \\
\hline $\begin{array}{c}\text { Nonverbal LD } \\
\text { (lower Performance IQ) }\end{array}$ & 9 \\
& $\begin{array}{c}\text { Reading \& Spelling: } 3 \\
\text { Math: } 5\end{array}$ \\
Written Language: 6
\end{tabular}


Table 4

Group Mean Scores and Standard Deviations

ADHD

\begin{tabular}{|l|r|r|}
\hline Subtest & \multicolumn{1}{|c|}{ Mean } & \multicolumn{1}{|c|}{ SD } \\
\hline GDS Block 1 & .67 & .27 \\
\hline GDS Block 2 & .78 & .23 \\
\hline GDS Block 3 & .82 & .16 \\
\hline GDS Block 4 & .83 & .16 \\
\hline GDS Total ER & .77 & .15 \\
\hline GDS V TOTCR & 39.40 & 6.84 \\
\hline GDS V TOT CM & 10.40 & 16.22 \\
\hline GDS D TOT CR & 29.04 & 13.32 \\
\hline GDS D TOT CM & 36.08 & 66.05 \\
\hline Digit Span (DS) & 8.20 & 2.43 \\
\hline DS Forward & -.55 & .78 \\
\hline DS Backward & -.33 & 1.02 \\
\hline Knox Cube (KC) & 7.40 & 2.25 \\
\hline KC Surprise Hits & .64 & .57 \\
\hline KC Surprise Miss & .80 & .65 \\
\hline WRAML GMI & 91.08 & 13.38 \\
\hline W Design Mem & 7.72 & 2.88 \\
\hline W Finger/Window & 8.28 & 2.37 \\
\hline W Number/Letter & 6.60 & 2.29 \\
\hline W Picture Mem & 9.24 & 2.55 \\
\hline W Sentence Mem & 8.56 & 2.97 \\
\hline W Sound Symbol & 10.20 & 2.58 \\
\hline W Snd Sym Delay & .14 & .79 \\
\hline W Story Memory & 10.32 & 2.48 \\
\hline W Story Delay & -.058 & .83 \\
\hline W Story Recog & .016 & .80 \\
\hline W Verbal Lrng & 9.60 & 3.40 \\
\hline W Ver Lrng Del & -.22 & 1.27 \\
\hline W Visual Lrng & 10.04 & 2.85 \\
\hline W Vis Lrng Del & -.008 & .82 \\
\hline
\end{tabular}

LD/ADHD

\begin{tabular}{|c|c|}
\hline Mean & SD \\
\hline .59 & .27 \\
\hline .70 & .27 \\
\hline .74 & .26 \\
\hline .73 & .22 \\
\hline .69 & .23 \\
\hline 39.04 & 4.69 \\
\hline 20.76 & 30.29 \\
\hline 24.00 & 10.61 \\
\hline 47.04 & 63.91 \\
\hline 7.88 & 2.60 \\
\hline-.66 & .94 \\
\hline-.89 & .63 \\
\hline 7.32 & 1.99 \\
\hline 1.04 & .98 \\
\hline .96 & .79 \\
\hline 85.28 & 10.23 \\
\hline 7.64 & 3.43 \\
\hline 7.00 & 2.24 \\
\hline 6.64 & 2.12 \\
\hline 9.20 & 2.10 \\
\hline 8.16 & 2.34 \\
\hline 8.36 & 2.18 \\
\hline-.72 & .76 \\
\hline 9.52 & 2.52 \\
\hline-.24 & 1.02 \\
\hline .09 & .94 \\
\hline 8.84 & 2.53 \\
\hline-.85 & 1.02 \\
\hline 9.24 & 2.50 \\
\hline-.05 & .85 \\
\hline
\end{tabular}

TBI

\begin{tabular}{|c|c|}
\hline Mean & SD \\
\hline .64 & .24 \\
\hline .77 & .16 \\
\hline .83 & .16 \\
\hline .85 & .14 \\
\hline .76 & .15 \\
\hline 41.13 & 8.55 \\
\hline 9.88 & 13.42 \\
\hline 28.88 & 13.75 \\
\hline 16.42 & 24.53 \\
\hline 7.75 & 2.85 \\
\hline-.51 & .94 \\
\hline-.56 & .97 \\
\hline 6.68 & 2.30 \\
\hline .72 & .68 \\
\hline .56 & .58 \\
\hline 86.42 & 11.02 \\
\hline 7.28 & 2.79 \\
\hline 7.00 & 2.54 \\
\hline 6.38 & 1.74 \\
\hline 8.48 & 2.73 \\
\hline 7.60 & 2.45 \\
\hline 9.80 & 3.00 \\
\hline-.32 & 1.06 \\
\hline 8.88 & 2.26 \\
\hline-.46 & .79 \\
\hline-.25 & 1.12 \\
\hline 10.83 & 3.37 \\
\hline-.07 & 1.15 \\
\hline 9.04 & 2.56 \\
\hline-.36 & .84 \\
\hline
\end{tabular}

Control

\begin{tabular}{|r|r|}
\hline \multicolumn{1}{|c|}{ Mean } & \multicolumn{1}{c|}{ SD } \\
\hline .64 & .25 \\
\hline .83 & .21 \\
\hline .79 & .16 \\
\hline .81 & .18 \\
\hline .77 & .16 \\
\hline 42.08 & 6.08 \\
\hline 2.52 & 3.98 \\
\hline 33.44 & 11.43 \\
\hline 7.40 & 14.72 \\
\hline 9.60 & 2.75 \\
\hline-.25 & .89 \\
\hline .13 & .87 \\
\hline 9.20 & 2.20 \\
\hline .60 & .76 \\
\hline .60 & .65 \\
\hline 102.16 & 12.23 \\
\hline 10.60 & 2.92 \\
\hline 10.12 & 2.79 \\
\hline 7.84 & 2.76 \\
\hline 10.04 & 2.30 \\
\hline 9.48 & 2.38 \\
\hline 11.20 & 2.58 \\
\hline .43 & .86 \\
\hline 10.52 & 2.29 \\
\hline .11 & .71 \\
\hline .21 & .80 \\
\hline 11.72 & 3.42 \\
\hline .54 & 1.05 \\
\hline 10.48 & 2.38 \\
\hline .32 & .90 \\
\hline
\end{tabular}

Total

\begin{tabular}{|c|c|}
\hline Mean & SD \\
\hline .63 & .26 \\
\hline .77 & .22 \\
\hline .80 & .19 \\
\hline .80 & .18 \\
\hline .75 & .18 \\
\hline 40.40 & 6.68 \\
\hline 10.90 & 19.45 \\
\hline 28.84 & 12.60 \\
\hline 26.84 & 50.12 \\
\hline 8.36 & 2.72 \\
\hline-.49 & .89 \\
\hline-.41 & .95 \\
\hline 7.65 & 2.35 \\
\hline .75 & .77 \\
\hline .73 & .68 \\
\hline 91.28 & 13.41 \\
\hline 8.31 & 3.26 \\
\hline 8.11 & 2.77 \\
\hline 6.87 & 2.30 \\
\hline 9.24 & 2.46 \\
\hline 8.45 & 2.60 \\
\hline 9.89 & 2.76 \\
\hline-.12 & .97 \\
\hline 9.81 & 2.44 \\
\hline-.16 & .86 \\
\hline .02 & .93 \\
\hline 10.24 & 3.34 \\
\hline-.15 & 1.22 \\
\hline 9.70 & 2.61 \\
\hline-.02 & .87 \\
\hline
\end{tabular}


Table 5

$\underline{\text { ANOVA Table }}$

\begin{tabular}{|l|c|c|}
\hline \multicolumn{1}{|c|}{ Subtest } & F (3,98) & Significance \\
\hline GDS Block 1 & .34 & .80 \\
\hline GDS Block 2 & 1.42 & .24 \\
\hline GDS Block 3 & 1.03 & .38 \\
\hline GDS Block 4 & 2.07 & .11 \\
\hline GDS Total ER & 1.11 & .35 \\
\hline GDS V TOTCR & 1.16 & .33 \\
\hline GDS V TOTCM & $\mathbf{4 . 0 6}$ & $\mathbf{. 0 1}_{\mathbf{a}}$ \\
\hline GDS D TOTCR & 2.44 & .07 \\
\hline GDS D TOTCM & $\mathbf{3 . 4 8}$ & $\mathbf{. 0 2}_{\mathbf{a}}$ \\
\hline Digit Span (DS) & 2.53 & .06 \\
\hline DS Forward & .94 & .42 \\
\hline DS Backward & $\mathbf{5 . 7 1}$ & $\mathbf{. 0 0}_{\mathbf{a}}$ \\
\hline Knox Cube (KC) & $\mathbf{6 . 1 0}$ & $\mathbf{. 0 0}_{\mathbf{a}}$ \\
\hline KC Surprise Hits & 1.72 & .17 \\
\hline KC Surprise Misses & 1.92 & .13 \\
\hline WRAML GMI & $\mathbf{1 0 . 6 3}$ & $\mathbf{. 0 0}_{\mathbf{a}}$ \\
\hline W Design Memory & $\mathbf{6 . 5 2}$ & $\mathbf{. 0 0}_{\mathbf{a}}$ \\
\hline W Finger/Window & $\mathbf{8 . 7 0}$ & $\mathbf{. 0 0}_{\mathbf{a}}$ \\
\hline W Number/Letter & 2.12 & .10 \\
\hline W Picture Memory & 1.72 & .17 \\
\hline W Sentence Memory & 2.41 & .07 \\
\hline W Sound Symbol & $\mathbf{5 . 1 2}$ & .37 \\
\hline W Sound Symbol Delay & $\mathbf{8 . 3 4}$ & $\mathbf{. 0 0}_{\mathbf{a}}$ \\
\hline W Story Memory & 2.50 & $\mathbf{. 0 0}_{\mathbf{a}}$ \\
\hline W Story Delay & 2.10 & .06 \\
\hline W Story Recognition & 1.07 & .11 \\
\hline W Verbal Learning & $\mathbf{3 . 9 8}$ & $\mathbf{. 0 0}_{\mathbf{a}}$ \\
\hline W Ver Lrng Delay & $\mathbf{6 . 4 8}$ & .17 \\
\hline W Visual Learning & 1.72 & $\mathbf{. 0 5} \mathbf{a}$ \\
\hline W Vis Lrng Delay & $\mathbf{2 . 6 4}$ & \\
\hline & & \\
\hline
\end{tabular}

${ }^{\mathrm{a}}$ significant at $\mathrm{p}<.05$. 
Table 6

$\underline{\text { Canonical Correlation Functions }}$

\begin{tabular}{|l|c|c|c|c|c|}
\hline Function & $\begin{array}{c}\text { Canonical } \\
\text { Correlation }\end{array}$ & $\begin{array}{c}\text { \% of } \\
\text { Variance }\end{array}$ & $\begin{array}{c}\text { Cumulative } \\
\mathbf{\%}\end{array}$ & $\mathbf{F}^{a}$ & Significance \\
\hline $\mathbf{1}$ & $\mathbf{. 7 3 5 9 8}$ & $\mathbf{3 1 . 1 5 4 9 1}$ & $\mathbf{3 1 . 1 5 4 9 1}$ & $\mathbf{1 . 2 8 2 7 5 ( 1 - 9 )}$ & $\mathbf{. 0 1 6}$ \\
\hline 2 & .63985 & 18.27423 & 49.42913 & $1.08900(2-9)$ & .244 \\
\hline 3 & .57615 & 13.09860 & 62.52773 & $.98117(3-9)$ & .544 \\
\hline 5 & .54698 & 11.25401 & 73.78174 & $.90648(4-9)$ & .727 \\
\hline 6 & .48770 & 8.22698 & 82.00872 & $.81715(5-9)$ & .868 \\
\hline 7 & .45685 & 6.95303 & 88.96176 & $.75170(6-9)$ & .915 \\
\hline 8 & .44011 & 6.33265 & 95.29440 & $.66222(7-9)$ & .950 \\
\hline 9 & .30305 & 2.66584 & 97.96024 & $.46505(8-9)$ & .990 \\
\hline
\end{tabular}

${ }^{a}$ Numbers in parentheses indicate number of functions included in analysis. 
Table 7

$\underline{\text { Canonical Variate Analysis }}$

\begin{tabular}{|c|c|c|}
\hline VARIABLE SET 1 & CORRELATION $^{\mathrm{a}}$ & COEFFICIENT $^{\mathrm{b}}$ \\
\hline GDS Block 1 & .00661 & .17850 \\
\hline GDS Block 2 & .29653 & .90898 \\
\hline GDS Block 3 & .10474 & .60410 \\
\hline GDS Block 4 & -.23126 & -.59372 \\
\hline Total ER & .06426 & -1.06007 \\
\hline GDS V TOTCR & .69474 & .66962 \\
\hline GDS V TOTCM & -.58832 & -.08220 \\
\hline GDS D TOTCR & .12215 & -.14236 \\
\hline GDS D TOTCM & -.40005 & -.25156 \\
\hline Percent Variance & 12.89225 & \\
\hline Redundancy & 6.98340 & \\
\hline \multicolumn{3}{|l|}{ VARIABLE SET 2} \\
\hline Digit Span (DS) & .15079 & -.24052 \\
\hline DS Forward & .21216 & .05008 \\
\hline DS Backward & .24636 & .06680 \\
\hline Knox Cube (KC) & .51973 & .20264 \\
\hline KC Surprise Hits & .29859 & .06884 \\
\hline KC Surprise Misses & .16772 & .26142 \\
\hline WRAML GMI & .46812 & 1.58180 \\
\hline W Design Memory & .52391 & .11168 \\
\hline W Finger/Window & .26942 & -.24143 \\
\hline W Number/Letter & .43207 & .00075 \\
\hline W Picture Memory & .22496 & -.18767 \\
\hline W Sentence Memory & .30107 & -.15363 \\
\hline W Sound Symbol & .21899 & -.75293 \\
\hline W Snd Symbol Delay & .33024 & .47590 \\
\hline W Story Memory & .04788 & -1.11203 \\
\hline W Story Delay & .28319 & .39483 \\
\hline W Story Recognition & .30102 & .44482 \\
\hline W Verbal Learning & .36346 & .25036 \\
\hline W Verbal Lrng Delay & .23260 & -.38104 \\
\hline W Visual Learning & -.01133 & -.51564 \\
\hline W Visual Lrng Delay & -.00405 & -.17802 \\
\hline Percent Variance & 4.96909 & \\
\hline Redundancy & 9.17359 & \\
\hline
\end{tabular}

${ }^{a}$ Correlations between variable sets and significant canonical function.

${ }^{\mathrm{b}}$ Standardized canonical coefficients. 
TABLE 8

Pearson Product-Moment Correlations: Variable Set 1 (GDS Scores) vs. Set 2 (WRAML, Knox, Digit Span Scores) ${ }^{\mathrm{a}}$

\begin{tabular}{|c|c|c|c|c|c|c|c|c|c|}
\hline Subtest & Block 1 & Block 2 & Block 3 & Block 4 & $\begin{array}{c}\text { Total } \\
\text { ER }\end{array}$ & $\begin{array}{c}\mathrm{V} \\
\text { TOTCR }\end{array}$ & $\begin{array}{c}\mathrm{V} \\
\text { TOTCM }\end{array}$ & $\begin{array}{c}\text { D } \\
\text { TOTCR }\end{array}$ & $\begin{array}{c}\text { D } \\
\text { TOTCM }\end{array}$ \\
\hline Digit Span & .06 & .07 & .03 & .13 & .09 & $.23 *$ & -.20 & -.02 & -.06 \\
\hline DS Fwd & $-.20 *$ & -.01 & -.13 & -.02 & -.11 & .19 & -.12 & -.05 & .03 \\
\hline DS Bkd & .07 & .10 & .06 & .12 & .11 & $.27 *$ & $-.22 *$ & .15 & -.18 \\
\hline Knox Cube & .10 & .13 & -.04 & -.16 & .04 & $.25 *$ & -.18 & .13 & -.19 \\
\hline KC Sur Hits & .00 & .02 & -.01 & -.08 & -.01 & $.23 *$ & .02 & -.04 & .06 \\
\hline KC SurMiss & .10 & .12 & .16 & .14 & .16 & $.25^{*}$ & .00 & -.02 & .04 \\
\hline W GMI & .07 & .18 & .20 & .04 & .15 & $.32 * *$ & $-.30 *$ & $.25 *$ & -.11 \\
\hline W Des Mem & .03 & $.20 *$ & .18 & -.03 & .12 & .18 & $-.31 * *$ & $.24 *$ & $-.27 *$ \\
\hline W Fin/Win & .06 & .04 & -.03 & -.07 & .02 & $.23 *$ & -.20 & .14 & .01 \\
\hline W Num/Let & -.02 & .12 & .13 & .07 & .09 & $.36 * *$ & $-.25 *$ & .02 & -.06 \\
\hline W Pic Mem & .02 & .08 & .12 & -.02 & .06 & .14 & .03 & .11 & .02 \\
\hline W Sen Mem & .06 & $.24 *$ & $.22 *$ & .14 & .19 & $.23 *$ & $-.24 *$ & .18 & -.02 \\
\hline W Snd Sym & .19 & .16 & $.20 *$ & .12 & $.21 *$ & .19 & $-.21 *$ & .17 & -.07 \\
\hline W SS Delay & .13 & $.23 *$ & .19 & .12 & $.20 *$ & $.21 *$ & $-.23 *$ & .15 & -.15 \\
\hline W StoryMm & .18 & .07 & .08 & .01 & .13 & .09 & -.01 & .08 & .07 \\
\hline W Story DL & .15 & .09 & .12 & -.06 & .11 & .16 & -.13 & .03 & .07 \\
\hline W StoryRec & $.24 *$ & .07 & .05 & -.09 & .11 & $.22 *$ & -.16 & .13 & .03 \\
\hline W Ver Lrng & .01 & .11 & .11 & .01 & .07 & $.23 *$ & -.19 & .18 & -.14 \\
\hline W VerL DL & .03 & .18 & $.21 *$ & .11 & .15 & .11 & -.18 & $.25 *$ & -.18 \\
\hline W Vis Lrng & -.02 & .03 & .15 & .09 & .07 & .05 & -.11 & $.25 *$ & .02 \\
\hline W VisL DL & .03 & .01 & .15 & .03 & .06 & .05 & -.01 & $.26 *$ & .09 \\
\hline
\end{tabular}

${ }^{a}$ Boldened blocks indicate variables that were significant in the canonical correlation.

$* \mathrm{p} \leq .05, * * \mathrm{p} \leq .001$ 
Table 9

$\underline{\text { Pearson Product-Moment Correlations Within Variable Set } 2}$

\begin{tabular}{|c|c|c|c|c|c|c|c|c|c|c|c|c|c|c|c|c|c|c|c|c|}
\hline Subtest & $\begin{array}{l}\text { Dig } \\
\text { Spn } \\
\end{array}$ & $\begin{array}{c}\text { DS } \\
\text { Fwd }\end{array}$ & $\begin{array}{c}\text { DS } \\
\text { Bkd }\end{array}$ & $\begin{array}{l}\text { Knx } \\
\text { Cbe } \\
\end{array}$ & $\begin{array}{l}\text { KC } \\
\text { SH } \\
\end{array}$ & $\begin{array}{l}\text { KC } \\
\text { SM } \\
\end{array}$ & $\begin{array}{c}\text { W } \\
\text { GMI }\end{array}$ & $\begin{array}{c}\text { W } \\
\text { DM }\end{array}$ & $\begin{array}{c}\mathbf{W} \\
\text { F/W } \\
\end{array}$ & $\begin{array}{c}\mathbf{W} \\
\mathbf{N} / \mathbf{L}\end{array}$ & $\begin{array}{c}\text { W } \\
\text { PM }\end{array}$ & $\begin{array}{c}\text { W } \\
\text { SnM }\end{array}$ & $\begin{array}{c}\text { W } \\
\text { SSM }\end{array}$ & $\begin{array}{c}\text { W } \\
\text { SSD }\end{array}$ & $\begin{array}{c}\text { W } \\
\text { StM }\end{array}$ & $\begin{array}{c}\text { W } \\
\text { StD }\end{array}$ & $\begin{array}{c}\text { W } \\
\text { StR }\end{array}$ & $\begin{array}{c}\text { W } \\
\text { VRL }\end{array}$ & $\begin{array}{c}\text { W } \\
\text { VRD }\end{array}$ & $\begin{array}{c}\text { W } \\
\text { VSL }\end{array}$ \\
\hline \multicolumn{21}{|l|}{ Dig Spn } \\
\hline DS Fwd & $.72 * *$ & & & & & & & & & & & & & & & & & & & \\
\hline DS Bkd & $.65 * *$ & $.38 * *$ & & & & & & & & & & & & & & & & & & \\
\hline KnxCbe & $.28 *$ & .17 & $.27 *$ & & & & & & & & & & & & & & & & & \\
\hline KC SH & -.01 & .01 & -.02 & $.38 * *$ & & & & & & & & & & & & & & & & \\
\hline KC SM & .11 & .07 & -.05 & .15 & $.43 * *$ & & & & & & & & & & & & & & & \\
\hline W GMI & $.38 * *$ & $.31 *$ & $.44 * *$ & $.49 * *$ & .02 & .08 & & & & & & & & & & & & & & \\
\hline W DM & .13 & .08 & $.32 * *$ & $.36 * *$ & -.04 & -.12 & $.66 * *$ & & & & & & & & & & & & & \\
\hline W F/W & $.37 * *$ & $.26^{*}$ & $.43 * *$ & $.48 * *$ & .01 & .09 & $.52 * *$ & $.27 *$ & & & & & & & & & & & & \\
\hline W N/L & $.55 * *$ & $.52 * *$ & $.33 * *$ & $.26 *$ & .17 & $.21 *$ & $.60 * *$ & $.25 *$ & $.25 *$ & & & & & & & & & & & \\
\hline W PM & .10 & .06 & .00 & $.20 *$ & .04 & $.23 *$ & $.47 * *$ & $.23 *$ & .16 & $.26 *$ & & & & & & & & & & \\
\hline W SnM & $.26 *$ & .19 & .17 & $.34 * *$ & .05 & .05 & $.58 * *$ & $.21 *$ & .18 & $.58 * *$ & $.21 *$ & & & & & & & & & \\
\hline W SSM & $.21 *$ & .15 & $.33 * *$ & $.31 * *$ & -.03 & -.06 & $.68 * *$ & $.41 * *$ & $.21 *$ & $.28 *$ & .13 & $.41 * *$ & & & & & & & & \\
\hline W SSD & .17 & .16 & $.33 * *$ & $.39 * *$ & -.06 & -.06 & $.64 * *$ & $.44 * *$ & $.21 *$ & $.28 *$ & .08 & $.40 * *$ & $.88^{* * *}$ & & & & & & & \\
\hline W StM & .08 & .06 & .09 & $.21 *$ & .03 & .15 & $.57 * *$ & .17 & .15 & $.29 *$ & $.24 *$ & $.40 * *$ & $.35 * *$ & $.34 * *$ & & & & & & \\
\hline W StD & .14 & .20 & .08 & $.25 *$ & .10 & .12 & $.57 * *$ & .17 & $.20 *$ & $.38 * *$ & $.31 * *$ & $.39 * *$ & $.31 * *$ & $.34 * *$ & $.80 * *$ & & & & & \\
\hline W StR & .12 & .02 & $.26 *$ & .17 & .06 & -.01 & $.37 * *$ & .18 & .12 & .16 & .08 & $.29 *$ & $.33 * *$ & $.29 *$ & $.60 * *$ & $.60 * *$ & & & & \\
\hline W VRL & .14 & .16 & $.31 * *$ & $.27 *$ & -.08 & -.08 & $.58 * *$ & $.39 * *$ & $.27 *$ & .16 & .13 & .09 & $.49 * *$ & $.45 * *$ & $.22 *$ & $.24 *$ & .04 & & & \\
\hline W VRD & .16 & .15 & $.34 * *$ & $.26^{*}$ & -.03 & .00 & $.58 * *$ & $.41 * *$ & .20 & $.21 *$ & .18 & .10 & $.48 * *$ & $.48 * *$ & $.24 *$ & $.31 * *$ & .16 & $.75 * *$ & & \\
\hline W VSL & $.21 *$ & .15 & $.33 * *$ & $.25 *$ & -.01 & .00 & $.63 * *$ & $.49 * *$ & $.33 * *$ & .20 & .18 & .19 & $.40 * *$ & $.38 * *$ & $.20 *$ & $.22 *$ & .14 & $.34 * *$ & $.45^{* * *}$ & \\
\hline W VSD & .19 & .09 & $.28 * *$ & .19 & -.06 & .06 & $.60 * *$ & $.46^{* * *}$ & $.38 * *$ & $.27 *$ & $.29 *$ & $.24 *$ & $.34 * *$ & $.32 * *$ & $.23 *$ & $.27 *$ & .16 & $.24 *$ & $.36^{* * *}$ & $.81 * *$ \\
\hline
\end{tabular}

* $\mathrm{p}<.05$.

$* * \mathrm{p}<.001$. 
Table 10

$\underline{\text { Summary of Canonical Discriminant Functions }}^{\mathrm{a}}$

\begin{tabular}{|c|c|c|c|c|c|}
\hline Function & $\begin{array}{c}\text { \% of } \\
\text { Variance }\end{array}$ & $\begin{array}{c}\text { Cumulative } \\
\text { \% }\end{array}$ & $\begin{array}{c}\text { Chi-square }^{\mathbf{a}} \\
\text { Df }\end{array}$ & Significance \\
\hline 1 & 59.6 & 59.6 & $65.461(1-3)$ & 27 & .000 \\
\hline 2 & 23.4 & 83.0 & $28.509(2-3)$ & 16 & .027 \\
\hline 3 & 17.0 & 100.0 & $12.139(3-3)$ & 7 & .096 \\
\hline
\end{tabular}

${ }^{a}$ Numbers in parentheses indicate number of functions included in analysis. 
Table 11

$\underline{\text { Structure Matrix }}^{\mathrm{a}}$

\begin{tabular}{|l|c|c|c|}
\hline & \multicolumn{3}{|c|}{ Function } \\
\cline { 2 - 4 } & $\mathbf{1}$ & $\mathbf{2}$ & $\mathbf{3}$ \\
\hline GDS V TOTCR & .189 & -.058 & .344 \\
\hline GDS V TOTCM & -.451 & .290 & -.249 \\
\hline GDS D TOTCM & -.329 & .223 & -.554 \\
\hline Knox Cube OH & .537 & .458 & .112 \\
\hline W GMI & .794 & .179 & .176 \\
\hline W Design Mem & .570 & .411 & .275 \\
\hline W Number/Letter & .308 & .249 & .164 \\
\hline W Snd Sym Delay & .729 & -.290 & -.176 \\
\hline W Verbal Lrng & .377 & -.189 & .562 \\
\hline
\end{tabular}

${ }^{a}$ Pooled within-groups correlations between discriminating variables and standardized canonical discriminant functions. 
Table 12

$\underline{\text { Functions at Group Centroids }}^{\mathrm{a}}$

\begin{tabular}{|l|c|c|}
\hline \multirow{2}{*}{ Group } & \multicolumn{2}{|c|}{ Function } \\
\cline { 2 - 3 } & $\mathbf{1}$ & $\mathbf{2}$ \\
\hline ADHD & .156 & -.331 \\
\hline TBI & -.452 & -.528 \\
\hline LD/ADHD & -.778 & .559 \\
\hline Control & 1.038 & .258 \\
\hline
\end{tabular}

${ }^{a}$ Unstandardized, significant canonical discriminant functions evaluated at group means. 
Table 13

$\underline{\text { Post-Hoc Scheffe Comparisons }}^{\mathrm{a}}$

\begin{tabular}{|c|c|c|c|c|}
\hline $\begin{array}{l}\text { DEPENDENT } \\
\text { VARIABLE }\end{array}$ & GROUP & ADHD & LD/ADHD & TBI \\
\hline \multirow{3}{*}{$\begin{array}{l}\text { GDS Vigilance } \\
\text { Total Comm. }\end{array}$} & LD/ADHD & -10.36 & & \\
\hline & TBI & .53 & 10.89 & \\
\hline & CONTROL & 7.88 & $18.24 *$ & 7.36 \\
\hline \multirow{3}{*}{$\begin{array}{l}\text { GDS } \\
\text { Distractibility } \\
\text { Total Comm. }\end{array}$} & LD/ADHD & -10.96 & & \\
\hline & TBI & 19.66 & 30.62 & \\
\hline & CONTROL & 28.68 & $39.64^{*}$ & 9.02 \\
\hline \multirow{3}{*}{$\begin{array}{l}\text { Knox Cube } \\
\text { Overall Hits }\end{array}$} & LD/ADHD & -.08 & & \\
\hline & TBI & .72 & .64 & \\
\hline & CONTROL & $-1.8^{*}$ & $-1.88^{*}$ & $-2.52^{*}$ \\
\hline \multirow{3}{*}{$\begin{array}{l}\text { WRAML } \\
\text { GMI }\end{array}$} & LD/ADHD & 5.8 & & \\
\hline & TBI & 4.66 & -1.14 & \\
\hline & CONTROL & $-11.08^{*}$ & $-16.88^{*}$ & $-15.74 *$ \\
\hline \multirow{3}{*}{$\begin{array}{l}\text { WRAML } \\
\text { Design Memory }\end{array}$} & LD/ADHD & -.08 & & \\
\hline & TBI & .44 & .36 & \\
\hline & CONTROL & $-2.88^{*}$ & $-2.96^{*}$ & $-3.32 *$ \\
\hline \multirow{3}{*}{$\begin{array}{l}\text { WRAML } \\
\text { Sound Symbol } \\
\text { Delay }\end{array}$} & LD/ADHD & $.87 *$ & & \\
\hline & TBI & .46 & -.41 & \\
\hline & CONTROL & -.28 & $-1.15^{*}$ & $-.74 *$ \\
\hline \multirow{3}{*}{$\begin{array}{l}\text { WRAML } \\
\text { Verbal } \\
\text { Learning }\end{array}$} & LD/ADHD & .76 & & \\
\hline & TBI & -1.23 & -1.99 & \\
\hline & CONTROL & -2.12 & $-2.88^{*}$ & -.89 \\
\hline
\end{tabular}

a Scores reflect mean differences between group scores.

* Significant at $\mathrm{p}<.05$. 
Table 14

Classification Matrix $^{\mathrm{a}}$

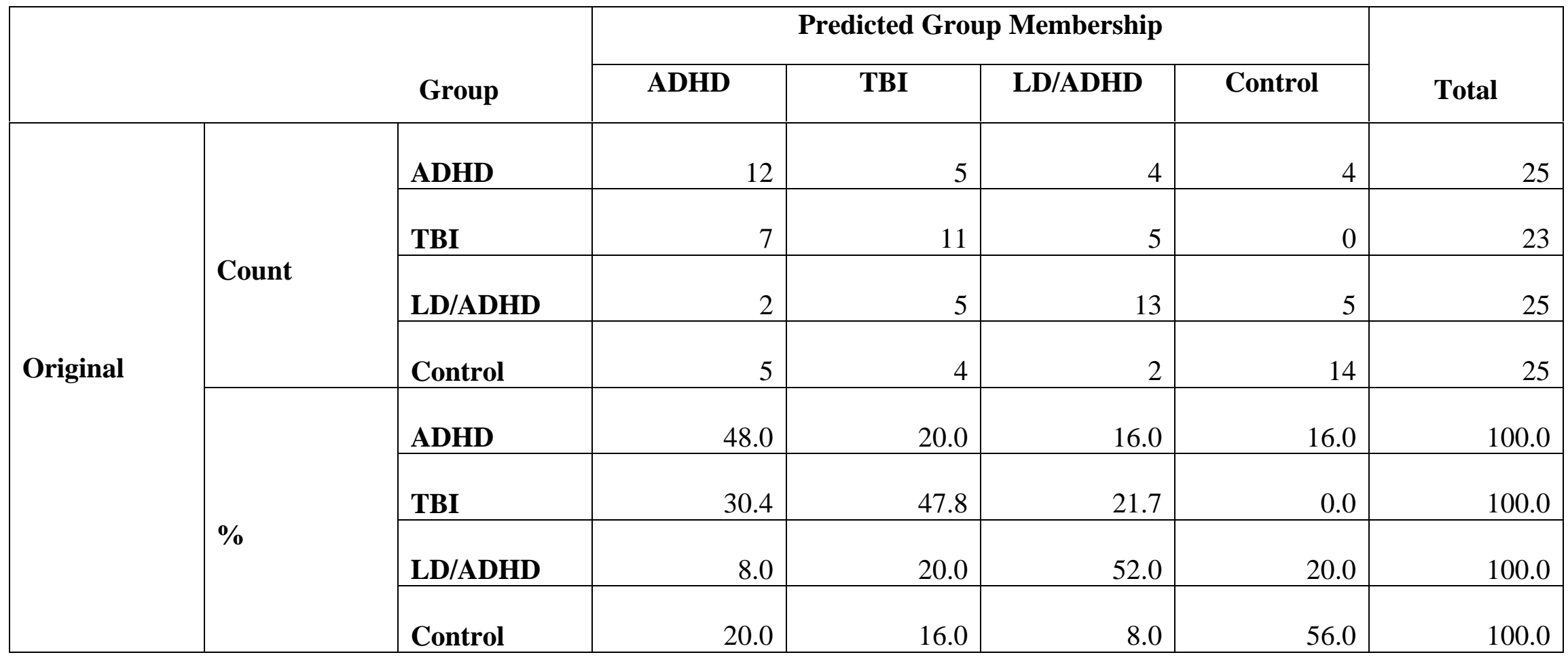

a $51.0 \%$ of original grouped cases correctly classified. 


\section{Figure Caption}

Figure 1. Plots of four group centroids on two discriminant functions. 


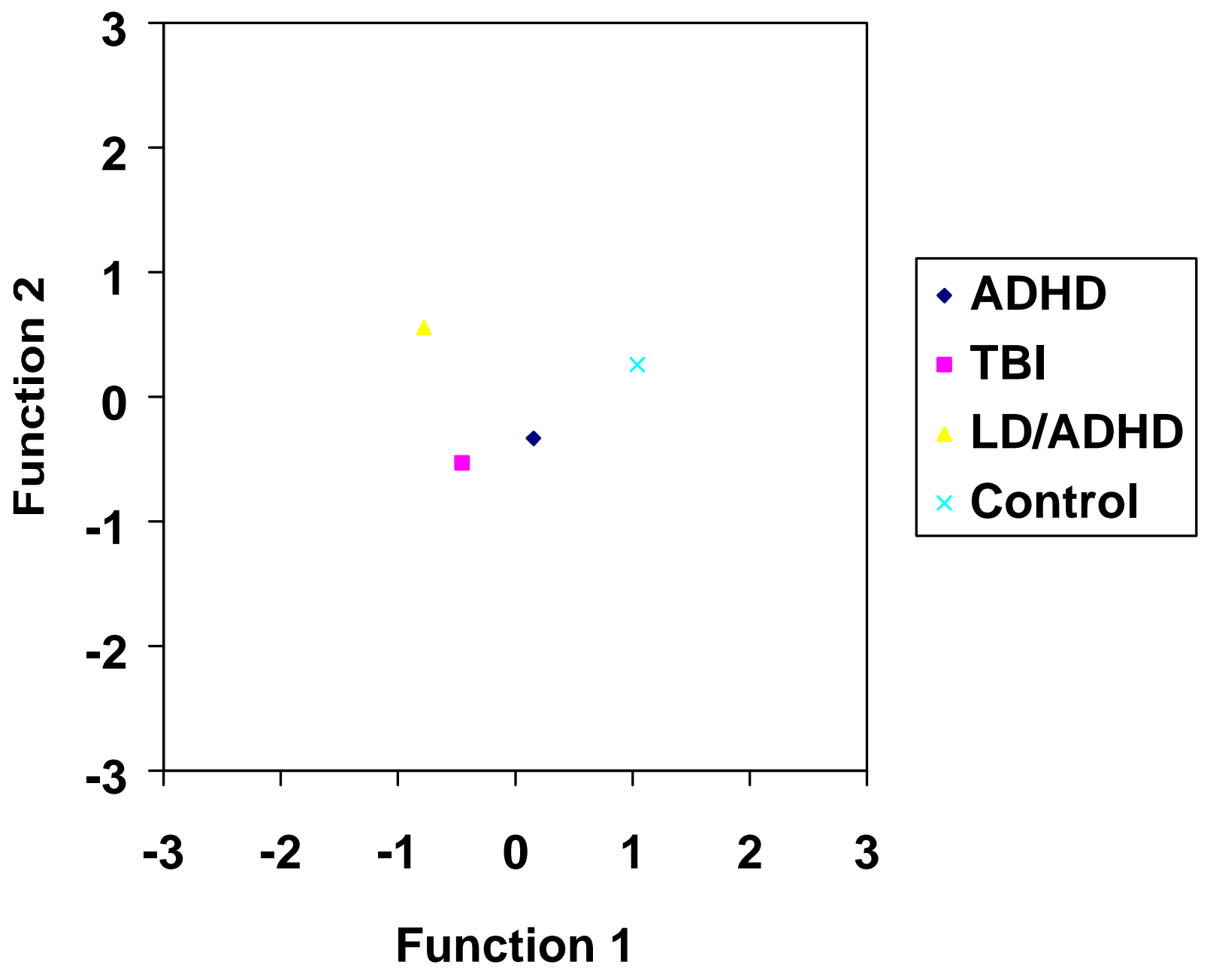




\section{VITA}

\section{KAREN S. KIRK}

BUSINESS ADDRESS: Heidelberg District Superintendent's Office Dept. of Defense Dependents' Schools

Unit 29237

APO AE 09102 (Germany)

011-49-6221-768081

\section{EDUCATIONAL HISTORY:}

- West Virginia University, PhD in Child Clinical Psychology, 1999

- University of Kentucky, MS in Education, 1988

- University of North Carolina - Chapel Hill, BA with Honors in Psychology, 1980

\section{POSITIONS HELD:}

DoDDS European Area Autism Consultant

Provide training and consultation to teachers, administrators, and parents across

8 DoDDS school districts (Iceland to Bahrain).

Lecturer, University of Maryland University College (Overseas)

Undergraduate psychology classes in child and adolescent psychology.

Behavior Management Consultant, DoDDS - Brussels District

Provided training and consultation regarding functional behavior assessment and positive behavioral support to elementary, middle, and high school students and teachers.

Supervising Psychoeducational Therapist, Wilmington TEACCH Center

Provided evaluative, therapeutic, and consultative services for clients with autism and related disabilities, and their families.

Staff Psychologist, Center for Autism and Related Disorders, Kennedy Krieger Institute Conducted assessments for individuals with suspected autism spectrum disorders.

Provided individual therapy, family programming, and social skills group training.

\section{PROFESSIONAL AFFILIATIONS:}

- American Association for Mental Retardation

- American Psychological Association, Divisions 16, 33, 40

- Autism Society of America

- International Neuropsychological Society

- National Academy of Neuropsychology 